\title{
ELUCIDATION OF THE PROTECTIVE MECHANISM OF of a CRYSTALLIN B in CARDIOMYOCYTES
}

by

Roxana Chis

A thesis submitted in conformity with the requirements for the degree of Masters of Science

Department of Physiology

University of Toronto

(C) Copyright by Roxana Chis 2012 


\title{
ELUCIDATION OF THE PROTECTIVE MECHANISM OF of $\alpha$ CRYSTALLIN B in CARDIOMYOCYTES
}

\author{
Roxana Chis \\ Masters of Science \\ Department of Physiology \\ University of Toronto
}

2012

\section{ABSTRACT}

$\alpha$-Crystallin B (cryAB) is the most abundant small heat shock protein in cardiomyocytes (CMs), where it has been shown to have potent anti-apoptotic properties. The mechanism by which cryAB prevents apoptosis has not been fully characterized. Therefore, I was interested in elucidating its protective mechanism in CMs. I identified its sub-cellular localization and its binding interactors following $\mathrm{H}_{2} \mathrm{O}_{2}$ exposure. I found that cryAB is found in the cytosol under control conditions and that following $\mathrm{H}_{2} \mathrm{O}_{2}$ exposure it becomes phosphorylated and translocates to the mitochondria. CryAB silencing resulted in increased apoptosis levels in CMs. Coimmunoprecipitation revealed an apparent increased interaction of cryAB and PcryAB with mitochondrial VDAC, caspase 12 and uncleaved caspase 3 in stressed hearts relative to controls. These results suggest that the cardio-protective effects of cryAB are mediated by its translocation to the mitochondria and its interaction with VDAC, caspase 12 and caspase 3 following exposure to $\mathrm{H}_{2} \mathrm{O}_{2}$. 


\section{ACKNOWLEDGEMENTS}

I would like to thank my supervisor, Dr. Anthony Gramolini, for all his guidance and support during the past two years. Dr. Gramolini, your intelligence has yet to cease to amaze me. You have profoundly augmented my expectations of others and of myself. I am very grateful to you for providing me with a different measuring stick for my future achievements.

I would also like to thank our research team. Dr. Parveen Sharma and Dr. Nicolas Bousette have offered their constant support throughout my time in the lab. What has been a challenging journey would have been unimaginably more difficult without you, Parv and Nic.

I cannot forget my lab-mates -Thiru, Melissa, Tim, Wen-Ping, Tetsuaki, Aaron and Jake - thank you for your help and great advice.

I am grateful to my advisory committee: Dr. Backx and Dr. von Harsdorf and to Dr. Jurisiocova for joining my committee at a moment's notice and providing very important guidance and discussion. Dr. Backx, I cannot thank you enough for your encouraging words during my committee meetings and all your invaluable advice and suggestions to help me achieve my career goals.

Finally, I would like to acknowledge my funding support from the Department of Physiology and the Ontario Graduate Scholarship Program. 


\section{TABLE OF CONTENTS}

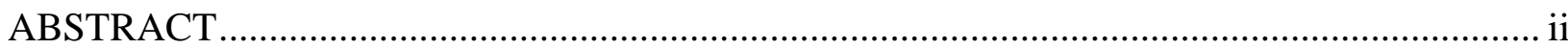

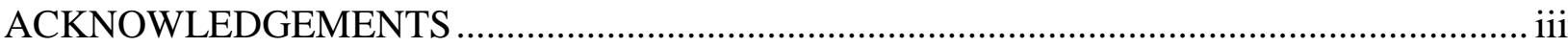

TABLE OF CONTENTS .................................................................................................... iv

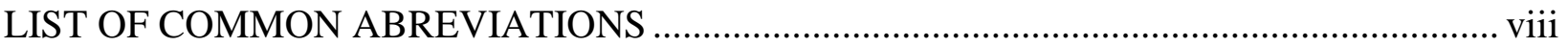

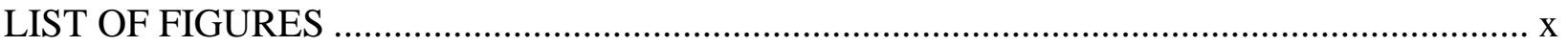

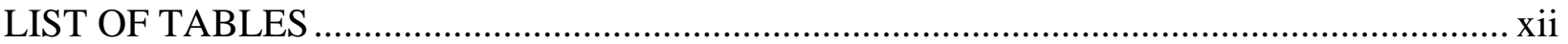

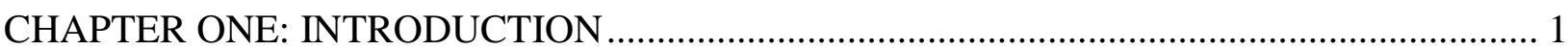

1.1 Cardiovascular Disease and Cardiomyocyte Loss ......................................................... 1

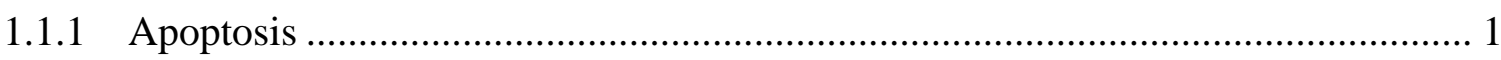

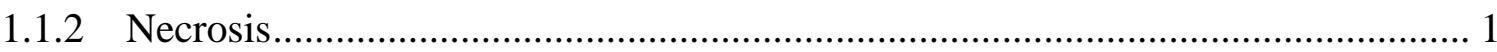

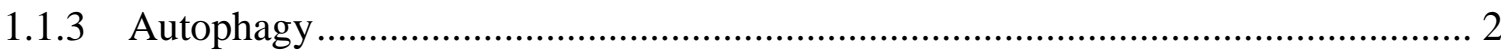

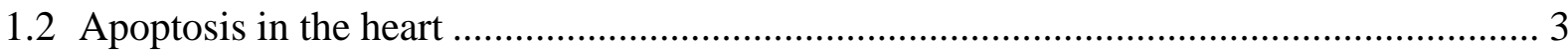

1.2.1 Apoptosis in ischemia/reperfusion injury ………............................................ 4

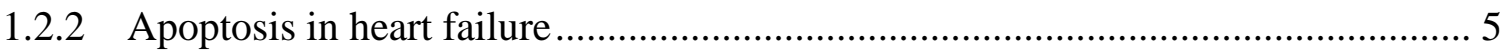

1.3 Apoptosis pathways in cardiomyocytes ..................................................................... 5

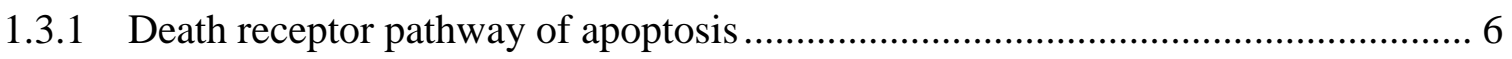

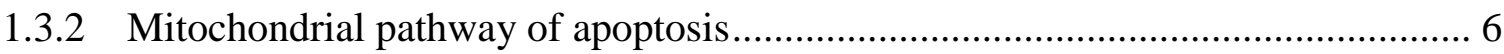

1.3.3 Cross-talk between the death receptor and mitochondrial pathways of

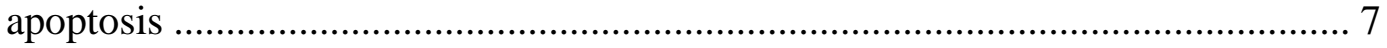

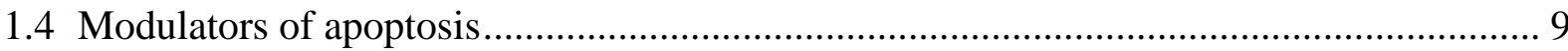

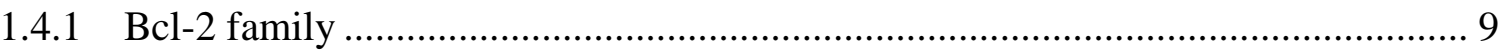

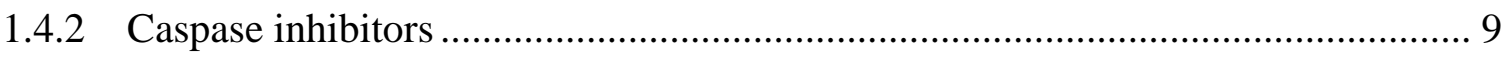

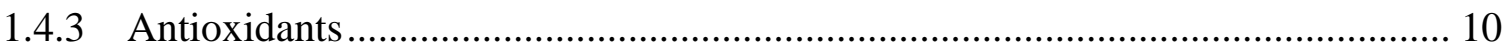

1.4.4 Heat shock proteins ................................................................................... 10

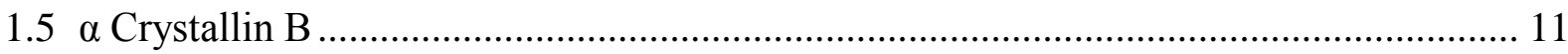




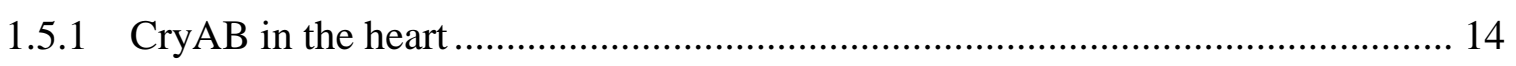

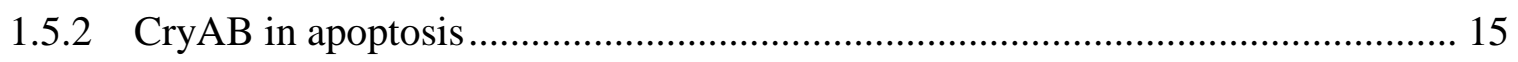

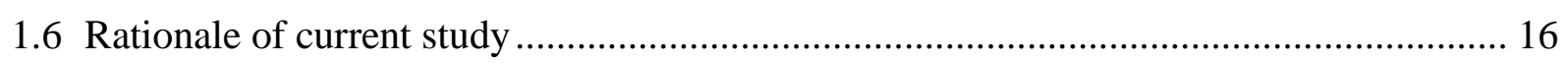

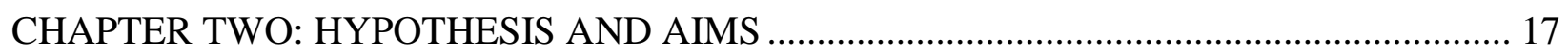

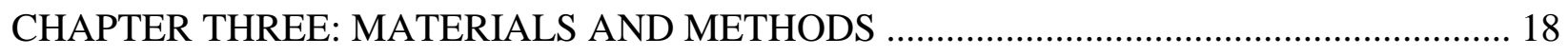

3.1 Lentivector Production and Transduction of Neonatal Cardiomyocytes ........................... 18

3.1.1 Chemical Transformation of DH5- $\alpha$ cells ......................................................... 18

3.1.2 Amplification and Maxi Preparations of cryAB cDNA ………………………... 19

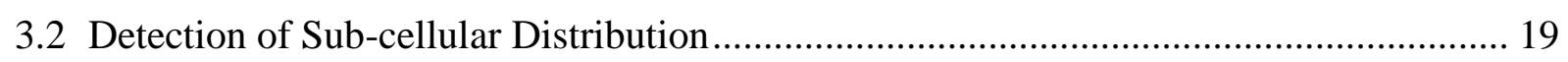

3.2.1 Sub-cellular Fractionation of Adult Mouse Hearts ................................................ 19

3.2.2 Sub-cellular Fractionation of Cultured Cardiomyocytes ..................................... 20

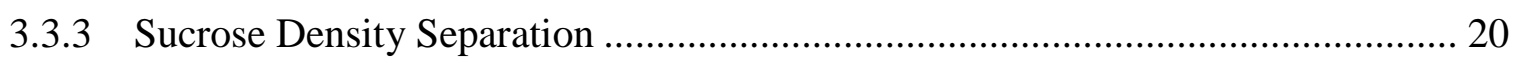

3.3 Immunoblot and Immunostaining Analysis .................................................................. 20

3.3.1 Immunoblot Detection of Sub-cellular Distribution of CryAB in Adult Whole

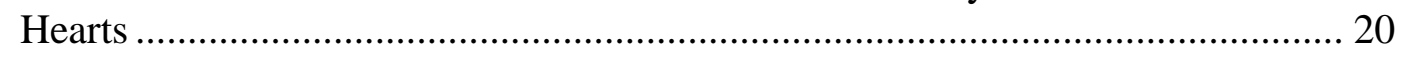

3.3.2 Immunoblot Detection of Sub-cellular Distribution of CryAB in Stressed Neonatal Cardiomyocytes ................................................................................. 21

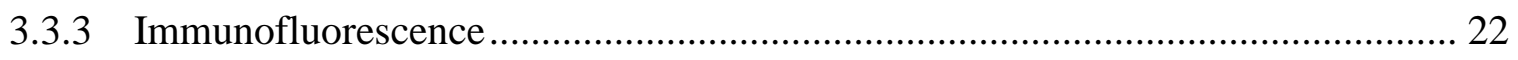

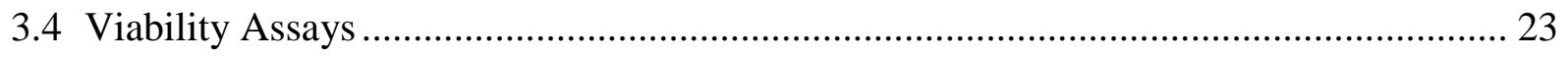

3.4.1 General Viability Assay ................................................................................ 23

3.4.2 Dissipation of Mitochondrial Membrane Potential Assay ..................................... 23

3.4.3 Caspase 3 Activity Assay ............................................................................ 24

3.4.4 TUNEL Assay ......................................................................................... 24

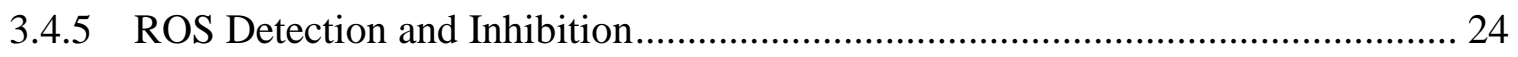

3.5 Co-Immunoprecipitation ..................................................................................... 24

CHAPTER FOUR: $\alpha$ CRYSTALLIN B INTERACTS WITH VDAC, CASPASE 3 AND CASPASE 12 TO PREVENT APOPTOSIS FOLLOWING $\mathrm{H}_{2} \mathrm{O}_{2}$ EXPOSURE IN

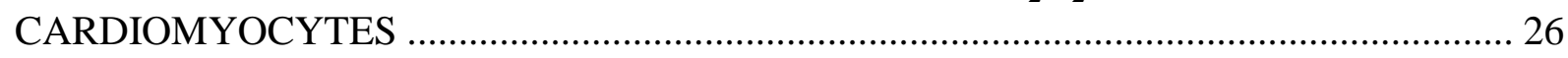




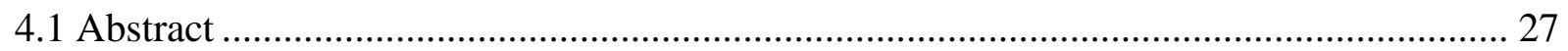

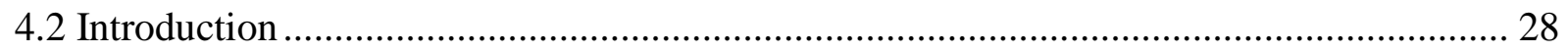

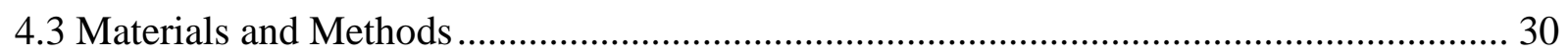

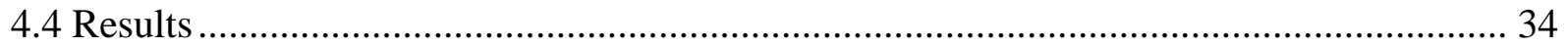

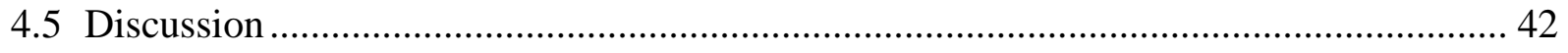

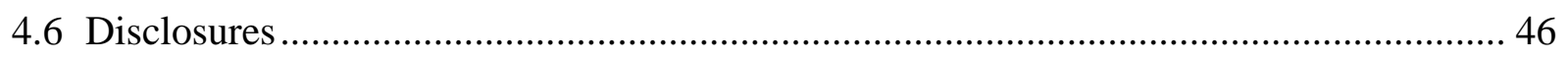

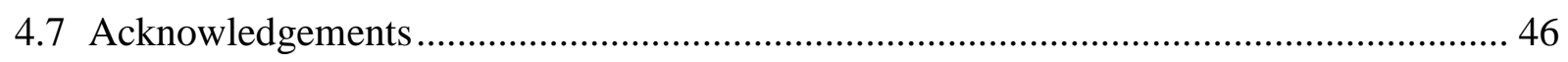

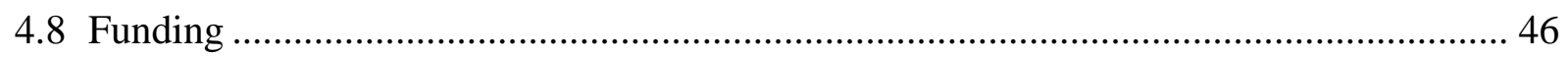

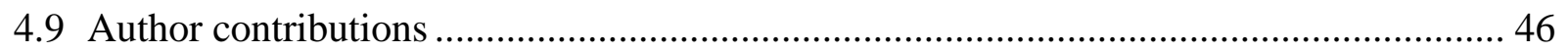

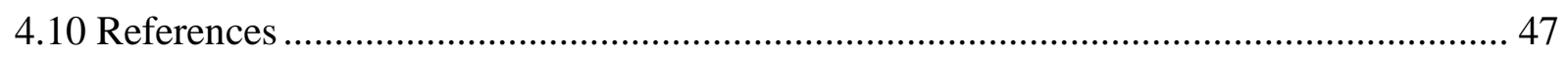

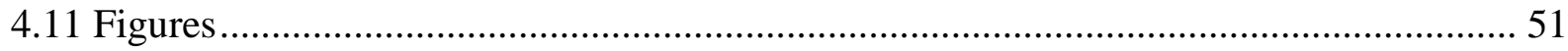

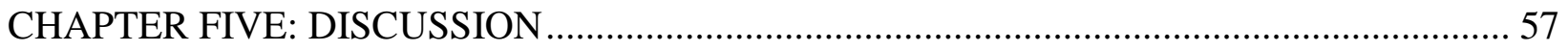

5.1 CryAB localization and translocation in cardiomyocytes ............................................... 57

5.1.1 Ischemia/Reperfusion injury and hydrogen peroxide in vitro ............................. 57

5.1.2 CryAB translocates from the cytosol to the mitochondria following oxaidative stress......................................................................................................... 58

5.2 CryAB silencing contributes to higher apoptosis levels in CMs ....................................... 60

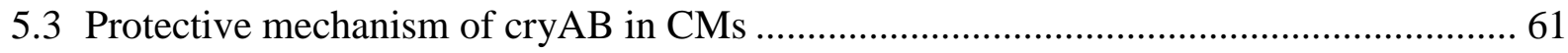

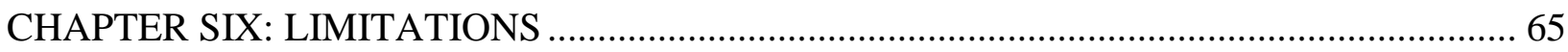

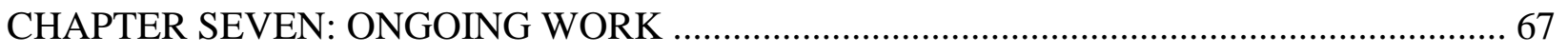

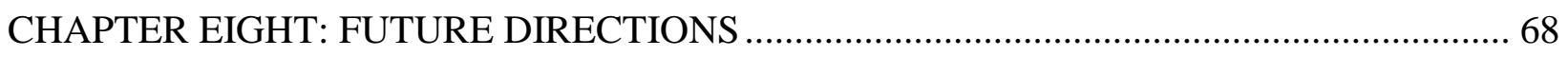

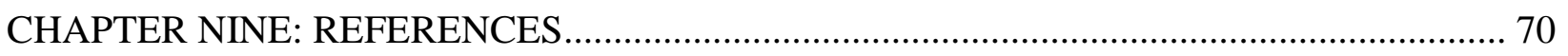

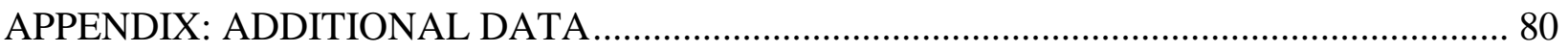

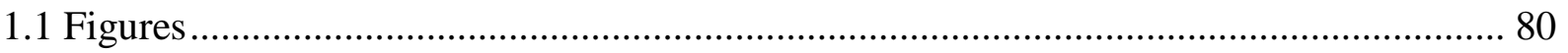

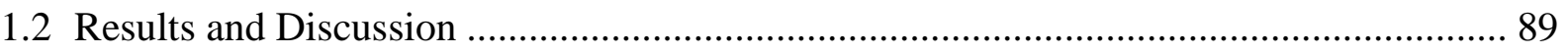

1.2.1 Over-expression of cry AB in mouse neonatal CMs .......................................... 89 


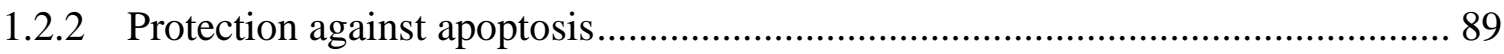

1.2.3 Optimization of $\mathrm{H}_{2} \mathrm{O}_{2}$ concentration for the induction of apoptosis .................... 90

1.2.4 Optimization of time-points for apoptosis measurements …………................... 90

1.2.5 Visual representation of cryAB localization and knock-down ............................. 91

1.2.6 Identification of cry $\mathrm{AB}$ protective mechanism and binding interactors in the intrinsic apoptotic cascade ............................................................................... 92 


\section{LIST OF COMMON ABREVIATIONS}

\begin{tabular}{|c|c|}
\hline Apaf-1 & Apoptotic Protease-Activating Factor-1 \\
\hline $\mathrm{ARC}$ & Apoptosis Repressor with Caspase Recruitment Domain \\
\hline ASK-1 & Apoptosis Signal-Regulating Kinase 1 \\
\hline Bad & Bcl-2 Associated Death Promoter \\
\hline Bak & Bcl-2 Homologous Antagonist/Killer \\
\hline Bcl-2 & B-cell Lymphoma 2 \\
\hline Bid & BH3 Interacting Domain \\
\hline $\mathrm{Ca} 2+$ & Calcium \\
\hline $\mathrm{CM}$ & Cardiomyocyte \\
\hline CNA & Calcineurin \\
\hline Co-Ip & Co-immunoprecipitation \\
\hline cryAB & Alpha Crystallin B \\
\hline $\operatorname{Daxx}$ & Death-Associated Protein 6 \\
\hline DNA & Deoxyribonucleic Acid \\
\hline ER & Endoplasmic Reticulum \\
\hline FADD & Fas-Associated via Death Domain \\
\hline Fas & Apoptosis Stimulating Fragment \\
\hline FBS & Fetal Bovine Serum \\
\hline GAPDH & Glyceraldehyde-3-Phosphate Dehydrogenase \\
\hline GFP-LC3 & Green Fusion Protein Light Chain 3 \\
\hline $\mathrm{H}_{2} \mathrm{O}_{2}$ & Hydrogen Peroxide \\
\hline hsp & Heat Shock Protein \\
\hline $\mathrm{I} / \mathrm{R}$ & Ischemia/Reperfusion \\
\hline IAP & Inhibitor of Apoptosis \\
\hline JNK & c-Jun Terminal Kinase \\
\hline KD & Knockdown \\
\hline
\end{tabular}




$\begin{array}{ll}\text { MAP kinase } & \text { Mitogen-Activated Protein } \\ \text { MPTP } & \text { Mitochondrial Permeability Transition Pore } \\ \text { Na/K ATPase } & \text { Sodium Potassium Adenosine Triphosphatase } \\ \text { NF-kB } & \text { Nuclear Factor Kappa B } \\ \text { PARP } & \text { Poly ADP-Polymerase Ribose } \\ \text { PcryAB } & \text { Phosphorylated (serine 59) Alpha Crystallin B } \\ \text { PDI } & \text { Protein Disulfide Isomerase } \\ \text { PLN } & \text { Phospholamban } \\ \text { RIP1 } & \text { Receptor Interacting Protein } \\ \text { ROS } & \text { Reactive Oxygen Species } \\ \text { SDS-page } & \text { Sodium Dodecyl Sulfate Polyacrylamide Gel Electrophoresis } \\ \text { ShRNA } & \text { Short Hairpin RNA } \\ \text { TNF } & \text { Tumor Necrosis Factor } \\ \text { TNFR1 } & \text { Tumor Necrosis Factor Receptor 1 } \\ \text { TOM 20 } & \text { Translocase of Outer Membrane 20 kDa } \\ \text { TRADD } & \text { Tumor Necrosis Factor Receptor-1-Associated Death Domain } \\ \text { VDAC } & \text { Voltage Dependent Anion Channel } \\ \text { WT } & \text { Wild-Type } \\ & \end{array}$




\section{LIST OF FIGURES}

Figure 1. Apoptotic Pathways in Cardiomyocytes. ................................................................. 8

Figure 2. Organization of human cryAB genomic and protein sequence................................ 14

Figure 3. CryAB distribution in cardiac cells. ............................................................... 51

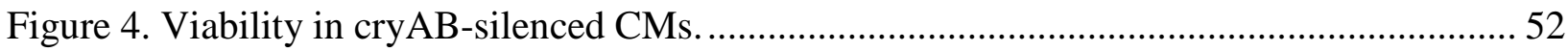

Figure 5. Upregulation and translocation of cryAB and PcryAB to the mitochondria under

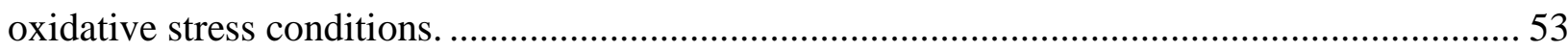

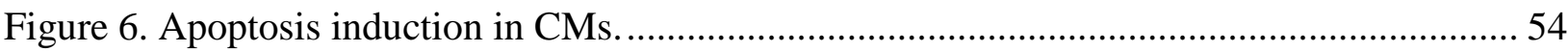

Figure 7. Effect of cryAB silencing on mitochondrial ultrastructure in mouse neonatal CMs.... 55

Figure 8. CryAB protein interactions in the apoptotic cascade. .......................................... 56

Figure 9. Schematic of cryAB protective mechanism.................................................... 64

Figure 10. CryAB expression following over-expression and in CNA CMs. .......................... 80

Figure 11. Mitotracker retention in CNA cardiomyocytes. ............................................... 81

Figure 12. Optimization of $\mathrm{H}_{2} \mathrm{O}_{2}$ concentration for induction of apoptosis in CMs. ................ 82

Figure 13. Time dependent dissipation of mitochondrial membrane potential and caspase 3

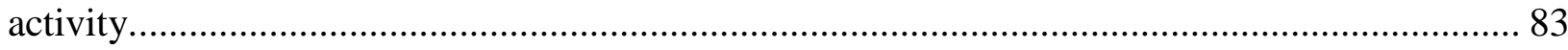

Figure 14. Translocation of cryAB and PcryAB to the mitochondria under oxidative stress

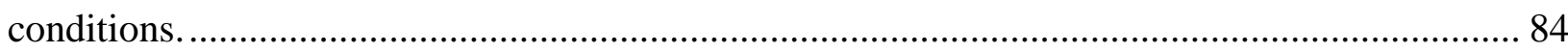

Figure 15. CryAB expression following stress and knock-down. ...................................... 85

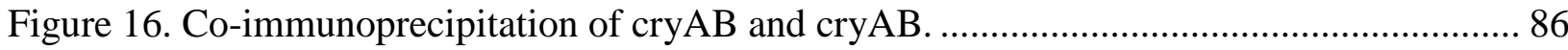

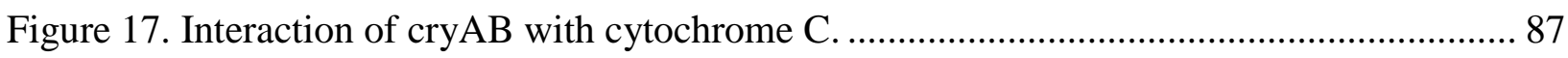


Figure 18. CryAB interactions in the apoptotic cascade that do not change following exposure to

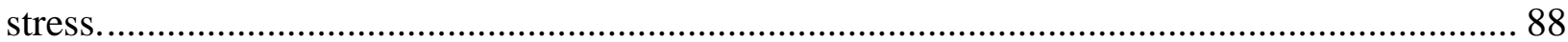




\section{LIST OF TABLES}

Table 1: Different types of cell death and their associated characteristics ................................ 3 


\section{CHAPTER ONE: INTRODUCTION}

\subsection{Cardiovascular Disease and Cardiomyocyte Loss}

Cardiovascular disease is the leading cause of death in the world ${ }^{1}$ and although the number of deaths from cardiovascular disease decreased by $21.9 \%$ during last two decades, it remains the

number one cause of death in North America ${ }^{1}$. Cardiovascular disease can be initiated by multiple factors and recently, evidence has accumulated that a major contributor to its initiation and progression is the loss of cardiomyocytes $(\mathrm{CMs})^{2}$. The adult myocardium is comprised of terminally differentiated $\mathrm{CMs}$, which are characterized by a significant loss of replicative potential or regeneration following injury ${ }^{3}$. Therefore, most cardiac insults lead to irreversible damage, which can contribute to the functional decline of the myocardium due to increased workload for the remaining myocardial cells, thus contributing to heart failure.

Cell death (including CM death) is attributed to at least three distinct processes - apoptosis, necrosis and autophagy, which have distinct histological and biochemical characteristics ${ }^{4}$.

\subsubsection{Apoptosis}

Apoptosis is a programmed cell death, a suicide, that is achieved by the activation of a cascade of events that culminate in cellular demise ${ }^{5}$. Apoptosis is a highly organized, energy-dependent and neat process, which does not cause damage to the surrounding tissue ${ }^{6}$. Apoptotic cell death is defined by particular characteristics - cell shrinkage, nuclear condensation and fragmentation, cleavage of chromosomal DNA and membrane blebbing ${ }^{7}$. Apoptosis is also marked by the absence of inflammation around the dying cell, since these are recognized and removed by phagocytic cells ${ }^{5}$. Furthermore, it has been shown that the underlying biochemical events that lead to the morphologic features of apoptosis result from the activation of a family of proteases, caspases (cysteine proteases), by either death receptor ligation or the release of apoptotic mediators from the mitochondria ${ }^{8}$.

\subsubsection{Necrosis}

In contrast to apoptosis, necrosis is a rapid, unregulated and irreversible process that occurs when cells are severely damaged. Necrosis involves swelling of the cell and its organelles, disruption of mitochondria, membrane breakdown cell lysis and an induction of inflammation around the 
dying cell attributable to the release of cellular contents and pro-inflammatory molecules ${ }^{6}$. Although the causative elements of necrosis are unclear, several mediators, organelles and cellular processes have been implicated in its occurrence. These include mitochondrial alterations, lysosomal changes, nuclear changes, lipid degradation ${ }^{9}$ and increases in the cytosolic concentration of calcium $\left(\mathrm{Ca}^{2+}\right)$ that result in mitochondrial overload and activation of calpains and cathepsins ${ }^{10}$. In several (but not all) cases of necrotic cell death, the serine/threonine kinase, Receptor Interacting Protein (RIP1) plays an important role ${ }^{11}$.

\subsubsection{Autophagy}

Autophagy is a distinct form of non-apoptotic death that is separate from necrosis. It is morphologically defined as a type of cell death that occurs in the absence of chromatin condensation but with significant autophagic vacuolization of the cytoplasm and with no association with phagocytes $^{12,13}$. Autophagy is characterized by massive sequestration of portions of the cytoplasm within autophagosomes for bulk degradation by lysosomes, giving the cell a characteristic vacuolated aspect ${ }^{14}$. Autophagosomes are bound by two membranes and contain degenerating cytoplasmic organelles or cytosol which allows them to be distinguished by transmission electron microscopy from other types of vesicles such as endosomes, lysosomes or apoptotic blebs ${ }^{4}$. The fusion between autophagosomes and lysosomes generates autolysosomes, in which both the autophagosome inner membrane and its luminal content are degraded by acidic lysosomal hydrolases. This catabolic process marks the completion of the autophagic pathway ${ }^{9}$. One technique commonly employed to detect autophagy relies on the redistribution of GFP-LC3 fusion proteins into vesicular structures ${ }^{15}$. However, doubts have been shed on the existence of cell death by autophagy in the mammals ${ }^{9}$, as in most cases described to date in which autophagy is suppressed by genetic knockout/knockdown of essential autophagy genes, cell death is not inhibited but rather occurs at an accelerated pace system, pointing towards autophagy as a cell survival mechanism ${ }^{5}$. Furthermore, there are only a few examples of cell death in model organisms that can be largely prevented by genetic inhibition of autophagy ${ }^{16,17}$. 
Table 1: Different types of cell death and their associated characteristics

The table below outlines the different types of cellular death and their characteristics.

\section{Type of cell death}

\begin{tabular}{|c|c|c|}
\hline Apoptosis & Necrosis & Autophagy \\
\hline $\begin{array}{l}\text { Programmed cell death } \\
\text { Reduction of cellular and nuclear } \\
\text { volume (pyknosis) } \\
\text { Nuclear fragmentation } \\
\text { (karyorrhexis) } \\
\text { DNA cleavage into } 200 \mathrm{bp} \\
\text { fragments } \\
\text { Plasma membrane blebbing } \\
\text { Engulfment by resident phagocytes }\end{array}$ & $\begin{array}{l}\text { Uncontrolled cell death } \\
\text { Cytoplasm swelling } \\
\text { (oncosis) } \\
\text { Rupture of plasma } \\
\text { membrane } \\
\text { Swelling of organelles } \\
\text { Chromatin condensation }\end{array}$ & $\begin{array}{l}\text { Cell survival or cell death } \\
\text { mechanism } \\
\text { Lack of chromatin } \\
\text { condensation } \\
\text { Significant vacuolization } \\
\text { of the cytoplasm } \\
\text { Presence of autophagic } \\
\text { vacuoles } \\
\text { No engulfment by } \\
\text { phagocytes }\end{array}$ \\
\hline
\end{tabular}

\subsection{Apoptosis in the heart}

It is now widely accepted that apoptosis occurs in the heart, however, its contribution to cardiac pathology remains controversial due to the difficulty of distinguishing between different types of cell death in cardiac tissue. This poses problems in quantifying the relative contribution of each to disease. Another obstacle to studying apoptosis of cardiomyocytes rests with determining the 'true' rate of apoptosis in disease. Some studies measured the value of apoptosis in the heart and found it to be very variable and in some cases very high ${ }^{18}$. However, different studies have provided seemingly low values $(<1 \%)^{19}$, attesting to the difficulty of measuring apoptosis rates accurately. The caveat is that such measurements represent only the number of cells undergoing apoptosis at a single point in time. It is now widely postulated that given the relatively short time course of apoptosis, the gradual loss of such numbers of myocardial cells could have serious consequences over an extended period. Thus, when investigating the role of apoptosis in heart 
disease, it is essential to consider not only the techniques used to detect and quantify apoptosis, but also the potential implications of such cell death over time.

A number of studies have detected apoptosis of CMs in different cardiac disease states and deemed its occurrence to be pathological in these cardiac diseases. Some clinical conditions in which increased apoptosis levels have been described in humans occur in the absence of heart failure - postnatal morphogenesis, acute myocardial infarction, cardiac allograft rejection and others occur in the presence of heart failure - ischemic cardiomyopathy, idiopathic dilated cardiomyopathy, acromegalic cardiomyopathy, diabetes/hypertension, arrhythmogenic right ventricular dysplasia, myocarditis, hypertrophic cardiomyopathy ${ }^{20}$.

\subsubsection{Apoptosis in ischemia/reperfusion injury}

Myocardial ischemia is caused by an inadequate blood supply, and thereby an inadequate supply of oxygen and glucose to the heart, leading to CM death if blood flow is not rapidly restored ${ }^{21}$. Reperfusion of ischemic tissue is a double edge sword, however, as it is also associated with cardiac tissue injury ${ }^{22}$. Although a large portion of the cell loss during cardiac ischemicreperfusion $(\mathrm{I} / \mathrm{R})$ occurs through necrosis, there is increasing evidence from studies conducted with several animal models of heart and on tissue from humans after myocardial infarction (a clinical counterpart of I/R injury) that $\mathrm{CM}$ death may also occur through apoptosis ${ }^{23,24,25}$. It has been reported in cases of regional ischemia such as myocardial infarction that the central area of infarct, or the most affected area by the stress, is predominantly necrotic, whereas apoptosis is found in the adjacent border areas ${ }^{26}$. Furthermore, recent evidence suggests that apoptosis contributes in part to overall myocyte cell death during the reperfusion period ${ }^{25}$. Another report confirms that the apoptotic cascade is triggered by ischemia and that reperfusion is required for completion of apoptosis ${ }^{27}$. The premise that apoptosis occurs in I/R injury is widely supported. In certain cases, the inhibition of apoptosis might prevent cell loss. For instance, cyclosporine, which inhibits apoptosis by blocking mitochondrial permeability-transition pores, can decrease the infarct size in patients with acute myocardial infarction ${ }^{28}$. However, whether apoptosis plays an important role in I/R injury remains unclear. Some studies suggests that the low level of cell death occurring by apoptosis means that it is not a decisive factor in tissue damage ${ }^{27}$. However, even if it is not found to be a decisive factor in initial tissue damage, it is possible that apoptosis in the border zone may contribute to the ongoing deterioration that may follow myocardial 
infarction. This is supported by the fact that apoptosis is found in the heart days after the initial reperfusion therapy ${ }^{26}$.

\subsubsection{Apoptosis in heart failure}

Several cardiac disorders, such as chronic overload such as that caused by hypertension or a defective heart valve, viral myocarditis, alcoholism, and ischemic heart disease culminate in heart failure ${ }^{6}$. In response to increased workload, the myocardium undergoes remodelling, which generally involves changes in gene expression and hypertrophy of CMs whereby the cells increase in $\operatorname{size}^{29}$. Although this structural change is an adaptive response to stress, it can eventually lead to heart failure. The progression to heart failure seems to be accompanied by apoptosis $^{30}$. Numerous studies support the possible role for apoptosis in heart failure. Examination of human heart tissue has shown apoptosis in association with conditions such as idiopathic dilated cardiomyopathy, ischemic cardiomyopathy, arrhythmogenic right ventricular dysplasia, and hypertrophic cardiomyopathy ${ }^{20}$. And although the degree of apoptosis found in these conditions is low, the gradual loss of CMs over time is thought to contribute to the eventual progression to heart failure. It has been proposed that apoptosis may have a role in the transition from mild to end-stage heart failure. In a recent study that measured the levels of proapoptotic Bax in relation to the severity of heart failure it was found that levels of Bax were significantly higher in association with mild heart failure compared with moderate or severe heart failure that CMs were more prone to apoptosis in the earlier stages of the disease thus facilitating the transition to heart failure ${ }^{31}$.

There is little doubt that apoptosis is found in association with myocardial disease given the increasing evidence that apoptosis is involved in heart failure. And although it may occur alongside other mechanisms of death modulation of apoptosis is of significant value.

\subsection{Apoptosis pathways in cardiomyocytes}

A number of different experimental models using different stresses, including work overload, hypoxia, neuro-humoral overstimulation, free radical stress, viral infection and toxic insults have have shown that $\mathrm{CM}$ can be induced to undergo apoptosis ${ }^{32}$. Apoptotic death occurs by two pathways - the activation of cell surface death receptors by extracellular ligands ${ }^{33}, 34$ and the 
activation of mitochondrial-related pro-apoptotic mechanisms in response to unfavorable changes in the intracellular environment ${ }^{35,36}$ (Fig.1).

\subsubsection{Death receptor pathway of apoptosis}

In the extrinsic or death receptor pathway of apoptosis, following ligand binding, death receptors interact with death domain adaptor proteins, such as Fas-Associated Death Domain (FADD), Tumor Necrosis Factor Receptor-1-Associated Death Domain (TRADD), Receptor-Interacting Protein (RIP), and Death-Associated Protein 6 (Daxx), which activate a cascade of cell signaling pathways (e.g., MAP kinase, $\mathrm{NF}-\kappa \mathrm{B}$ and $\mathrm{Akt}$ ) that regulate gene expression and the phosphorylation status of proteins (e.g., Bcl-2 proteins and IAP proteins) ${ }^{37}$. These, in turn, modulate the activity of different families of proteases (e.g., caspases, cathepsins and calpains) involved in the execution of the final steps of the apoptotic process ${ }^{33,38,39}$.

The death receptor pathway has been found to be activated in the heart. For instance, Fas and Fas ligand expression has been found in the heart ${ }^{40}$. Enhanced expression of Fas is found in association with increased apoptosis in experimental models of myocardial infarction ${ }^{41}$ hypoxia $^{42}$, and overstretched myocardium ${ }^{43}$. Signaling through the TNF receptor has also been reported in the heart. Like Fas, binding of the TNFR1 by its ligand (TNF- $\alpha$ ) results in the recruitment of adaptor proteins that transduce signals downstream, leading to various physiological events including inflammation, cell growth, differentiation, and apoptosis, depending on the different adaptor proteins and downstream signaling events associated with receptor activation ${ }^{33}$. Furthermore, it has been demonstrated that TNF plays a role in the progression of myocardial disease, with increased TNF- $\alpha$ and TNFR1 expression are found in association with heart failure ${ }^{44}$. Agents that suppress TNF- $\alpha$ have been shown to have positive therapeutic effects, thus attesting to the negative role of elevated TNF- $\alpha^{45}$. As TNF- $\alpha$ can induce apoptosis of $\mathrm{CMs}^{46}$, it is thought that at least part of its pathogenic effect in the heart is due to the induction of cell death.

\subsubsection{Mitochondrial pathway of apoptosis}

A second pathway of apoptosis that occurs in CMs is the intrinsic, or mitochondrial pathway. In addition to their established role in energy production, mitochondria are actively involved in the regulation of apoptosis ${ }^{49}$. In essence, stresses like ischemia have been shown to lead to 
mitochondrial death signaling, where the opening of the mitochondrial permeability transition pore (MPTP) leads to a change in the permeability of the inner mitochondrial membrane. MPTP is a mitochondrial inner membrane channel whose opening allows molecules smaller than 1500 Daltons to enter the mitochondrial matrix ${ }^{47}$. The entry of small molecules leads to increased mitochondrial permeability and ultimately to swelling of the mitochondria and rupture of the outer mitochondrial membrane, thus leading to the release into the cytosol of a number of highly lethal mitochondrial substances that can initiate apoptosis. One of these is the small electron transporter cytochrome $\mathrm{C}$ that forms an apoptosis-promoting complex, the apoptosome, with procaspase-9 and its cofactor apoptotic protease-activating factor-1 (Apaf-1), thus activating caspase- 9 and, subsequently, caspase- 3 and other caspases ${ }^{48}$. Mitochondria are a primary site of action of the apoptosis regulatory proteins of the Bcl-2 family and a major source of reactive oxygen species (ROS), which have been implicated in cellular damage and death ${ }^{49}$.

Recent studies suggest that cytochrome C-mediated apoptosis is important in CMs. Serum and glucose deprivation induce cytochrome $C$ release in vitro, resulting in activation of caspases-9 and -3 and apoptosis as determined by nuclear fragmentation, DNA cleavage, and processing of caspase substrates $^{50}$. As serum and glucose deprivation are components of ischemia in vivo, these results suggest that this pathway may be involved in cell death in relation to heart disease. ROS have also been implicated in $I / R$ induced damage, and it has been reported that mitochondrial cytochrome $\mathrm{C}$ release, activation of caspase-3, and PARP cleavage are involved in $\mathrm{H}_{2} \mathrm{O}_{2}$-induced cardiomyocyte apoptosis ${ }^{51}$. These in vitro studies demonstrate that $\mathrm{CM}$ apoptosis can occur via a cytochrome C-mediated pathway.

\subsubsection{Cross-talk between the death receptor and mitochondrial pathways of apoptosis}

In certain cases, the two pathways do not occur in isolation, but rather interactions between the two pathways may take place ${ }^{52}$. For example, the pro-apoptotic protein BH3 interacting-domain (Bid) is cleaved by caspase- 8 in response to Fas and TNF receptor activation ${ }^{53}$. Following cleavage, the $\mathrm{C}$-terminal fragment of Bid translocates and binds to the mitochondria, leading to the release of cytochrome $\mathrm{C}$ and activation of downstream caspases. Another instance of crosstalk is that of the apoptosis signal-regulating kinase 1 (ASK1), which is a member of the MAP kinase family. This activates JNK and p38 kinases leading to apoptosis mainly by mitochondriadependent caspase activation, due to the phosphorylation and inactivation of the anti-apoptotic 
protein $\mathrm{Bcl}-2$ that prevents the release of cytochrome $\mathrm{C}^{54}$. Thus, $\mathrm{Bcl}-2$ family proteins are mediators linking the death receptor signals to the mitochondria-dependent death signals ${ }^{55}$.

\section{Death Receptor Pathway}

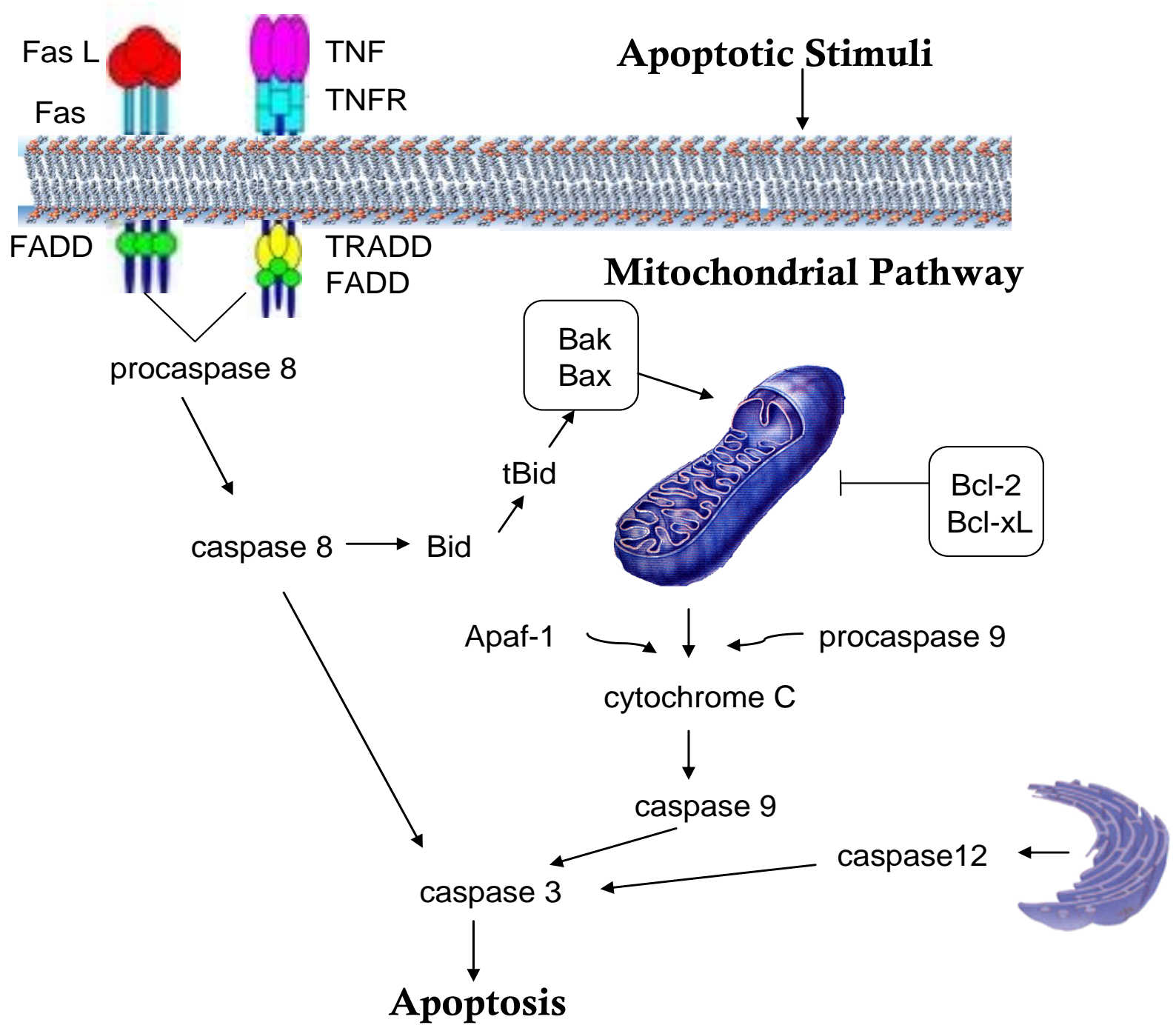

Figure 1. Apoptotic Pathways in Cardiomyocytes.

The extrinsic pathway occurs by ligand binding to the death receptor. Death receptors then interact with death domain adaptor proteins, which activate a cascade of cell signaling pathways (e.g., MAP kinase, NF-kB and Akt) that regulate gene expression and the phosphorylation status of proteins (e.g., Bcl-2 proteins and IAP proteins). These, in turn, modulate the activity of caspases, cathepsins and calpains. The intrinsic pathway is stimulated by mitochondrial dysfunction. Here, apoptosis-inducing signals, such as I/R, hypoxia or oxidative stress cause the opening of the mitochondrial permeability transition pore (MPTP). Opening of the pore leads to dissipation of mitochondrial membrane potential and matrix swelling, which ruptures the outer mitochondrial membrane, leading to the release of proteins from the intermembrane space, including cytochrome $\mathrm{C}$. This causes the activation of caspase-9, which in turn activates caspase-3. ER stress activates caspase 12, which also activates caspase 3, leading to apoptosis. Various intermediary signaling and proteins inhibiting the apoptotic cascade are also shown. Apaf-1, apoptosis-activating factor 1; Bak, bacille Calmette-Guérin; Bax, BCL-2-associated X protein; Bid, proapoptotic Bcl-2 family member; tBid, truncated beta interaction domain. 


\subsection{Modulators of apoptosis}

Apoptosis is a highly orchestrated multistep process with many different points at which it can be regulated. Regulation of apoptosis is particularly important in fully differentiated and replication-impaired CMs in order to avoid unnecessary death of salvageable cells. Regulation of apoptosis is also important in promoting its occurrence in response to irreversible cellular damage, as opposed to necrosis, which could further harm the myocardium. Hence, mechanisms allowing the modulation of death signals in CMs are important as they may help to keep the cell alive.

\subsubsection{Bcl-2 family}

CMs are known to express the Bcl-2 family of proteins and it has been suggested that these play a role in modulating apoptosis. A dramatic increase in Bax expression and decreased expression of Bcl-2 have been reported in the left ventricle in response to chronic pressure overload in the $\mathrm{rat}^{56}$, and it is thought that such changes are likely to influence cells to undergo apoptosis. In a model of ROS-induced apoptosis, $\mathrm{H}_{2} \mathrm{O}_{2}$ led to increased expression of pro-apoptotic $\mathrm{Bad}$ and elicited the translocation of $\mathrm{Bax}$ and $\mathrm{Bad}$ to the mitochondria, resulting in cytochrome $\mathrm{C}$ release, activation of caspase-3, and cleavage of $\mathrm{PARP}^{51}$. An up-regulation of the $\mathrm{Bcl}-2$ protein family has also been reported in human end-stage heart failure where increases in pro-apoptotic Bak and Bax and anti-apoptotic Bcl-2 were observed in association with apoptosis ${ }^{57}$. Expression of Bax was significantly higher than that of the anti-apoptotic proteins, which suggests that in the failing human heart apoptosis is favoured.

Manipulation of the Bcl-2 system may also provide new treatments to prevent $\mathrm{CM}$ apoptosis. The potential strategies could involve up-regulating the anti-apoptotic Bcl-2 pathway or inhibiting pro-apoptotic pathways. Indeed, it has been shown that ischemic preconditioning with reduced apoptosis is associated with up-regulation of Bcl-2 in rats ${ }^{58}$ and that over-expression of Bcl-2 in ventricular myocytes prevents apoptosis ${ }^{59,60}$.

\subsubsection{Caspase inhibitors}

The final steps of apoptotic death are mediated by active caspases ${ }^{33,38,39}$. ARC (apoptosis repressor with caspase recruitment domain), an inhibitor of apoptosis that is expressed almost exclusively in skeletal and cardiac muscle has been shown to interact with caspases- 8 and -2 and 
to attenuate apoptosis induced by stimulation of death receptors ${ }^{61}$. The use of synthetic inhibitors of apoptosis presents another potential therapeutic avenue. broad range caspase inhibitors were effective in reducing myocardial reperfusion injury in rats, which was attributed in part to the attenuation of cardiomyocyte apoptosis ${ }^{62}$. The same inhibitor is reported to attenuate apoptosis in rabbit cardiomyocytes ${ }^{63}$. However, caspase inhibition has been stipulated to increase ROS production resulting in secondary toxicity ${ }^{64}$. Specific inhibitors of caspases also seem to have regulatory effects on apoptosis. One study found that CM DNA fragmentation and caspase activation were prevented by inhibitors of caspase-1 and -3 without reduction of the infarct size in $\mathrm{I} / \mathrm{R}$ rat hearts ${ }^{65}$. This contrasts with another study that found that inhibitors of caspase- $8,-9$, and -3 all limited infarct size as a result of reperfusion injury ${ }^{66}$. The different outcomes of these studies may reflect the different administration times of the inhibitors, before ischemia and during early reperfusion, respectively.

\subsubsection{Antioxidants}

It has been proposed that apoptosis is modulated by oxidative stress and antioxidants play a part in its prevention ${ }^{67}$. Cellular antioxidants act by removing free radicals from the cell and thereby minimize oxidative stress resulting from a variety of insults, including $I / R$ injury ${ }^{68}$. Several endogenous antioxidants are found in CMs including glutathione (GSH), glutathione peroxidase (GSHPx), superoxide dismutase (SOD) catalase, and vitamins $\mathrm{E}$ and $\mathrm{C}^{69}$. In relation to apoptosis, a drop in intracellular levels of GSH is associated with increased oxidative stress. This leads to damage of cellular macromolecules within the cell and subsequent cell death ${ }^{67}$. In the heart, GSHPx has a role in regulating I/R-induced apoptosis. Experiments with GSHPx knockout mice demonstrated increased levels of apoptosis in response to ischemia/reperfusion whereas mice overexpressing GSHPx were more resistant to such damage ${ }^{70}$. Over-expression of manganeseSOD (MnSOD) protects against I/R injury in transgenic mice, in part by modulating apoptosis ${ }^{71}$.

\subsubsection{Heat shock proteins}

It is well established that heat shock proteins (hsps) are synthesized in response to a variety of stressful stimuli and that their expression coincides with increased resistance to subsequent cellular damage is due at least in part to interference with apoptosis ${ }^{72}$. The hsps are recognized as important regulators of CM apoptosis. Different families of hsps are expressed in the heart, including the hsp90, 70, 60, 27, and 10 families; induction of these proteins by a variety of 
agents including cardiotrophin- $1^{73}$, heat shock, and ethanol ${ }^{73}$ protects CMs against stress such as ischemia. For instance, over-expression of hsps70 and 27 can protect cells against ceramide, serum withdrawal, and lethal hypoxia ${ }^{74}$, all of which are known to be inducers of apoptosis. Thus, hsps are likely to be key regulators of apoptosis in the heart during stressful conditions such as ischemia.

Although hsps play a key role in the regulation of apoptosis, the mechanisms by which they do so are not fully understood. It is known that hsp70 inhibits apoptosis downstream of cytochrome $\mathrm{C}$ release and upstream of caspase- 3 activation $^{75}$. Hsp27 can prevent cytochrome $\mathrm{C}$ release at the level of the mitochondria or can interact with cytochrome $\mathrm{C}$ or procaspase-3, preventing apoptosome formation and caspase- 3 activation, respectively ${ }^{76,77}$. Given the cardioprotective effect of heat shock protein induction in in vitro and animal studies, these proteins represent a potentially valuable treatment for cardiac disease.

\section{5 a Crystallin B}

Alpha-crystallin is a major protein component of the lens in the vertebrate eye, and a key member of the small heat-shock proteins with the 'alpha-crystallin domain' being a common Cterminal consensus sequence to all the members of the small heat-shock protein super family ${ }^{78}$. Alpha crystallin was initially discovered in the mammalian lens where $\sim 50 \%$ of the total dry mass of the lens is alpha-crystallin protein ${ }^{79}$. In the lens, crystallin is involved in maintaining the necessary refractive index of the lens ${ }^{80}$, and maintaining lens transparency ${ }^{79}$, through ensuring proper protein folding and minimizing protein aggregation. For instance, during aging, and especially in the center part of the lens, there is increased protein unfolding and denaturing. This can interfere with vision, through the formation of a cataract, for instance. Alpha-crystallin binds such unfolded or denatured proteins and suppresses non-specific aggregation ${ }^{80}$, thus maintaining lens transparency.

There are two alpha-crystallin genes, alpha A, and alpha B. In humans, the alps A gene is found on chromosome 21 and encodes for a 173 amino acid residue protein, while the alpha $\mathrm{B}$ gene is found on chromosome 11, is made up of 3124 base pairs organized within 3 exons and 2 introns (Fig. 2A) and encodes for a 175 amino-acid residue protein. The amino-acid sequence homology between alpha $\mathrm{A}$ and alpha $\mathrm{B}$ is about $57 \%^{80}$, and the expression pattern of alpha $\mathrm{A}$ and alpha $\mathrm{B}$ 
is also quite different. Alpha A crystallin is found mainly in the lens with trace amounts in other tissues $^{80}$, whereas alpha $\mathrm{B}$ is constitutively expressed in the lens of the eye , brain, kidney, skeletal and cardiac muscle ${ }^{81,82}$. In mammals, alpha crystallin B associates with alpha crystallin A to form large hetero-oligomeric structures ${ }^{82}$.

Alpha crystallin B (cryAB) or heat shock protein beta 5 (hspB5) is a $20 \mathrm{kDa}$ small heat shock protein ${ }^{83}$ and a molecular chaperone ${ }^{79}$. As a small hsp, cryAB is involved in modulating the ubiquitin-proteasome pathway and is essential for proper disassembly-assembly of protein complexes to prevent undesirable interactions and aggregation ${ }^{84}$. Like other small hsps cryAB can form globular oligomeric structures that are characterized, in mammalian cells, by molecular masses ranging from 50 to approximately $700-800 \mathrm{kDa}^{80}$ and it is this dynamic organization of small hsps oligomers that appears to be important in the control of their activity.

Another property of some small hsps, including cryAB, concerns their ability to modulate their activities in response to a wide variety of stimuli ${ }^{85,86}$, a process known to be dependent on phosphorylation. CryAB can be phosphorylated at three serine site corresponding to residues 19 , 45 and 59 in response to cellular stresses ${ }^{82}$ (Fig.2B). These phosphorylated serine sites are found in the N-terminal part of the polypeptides, in the WDPF domain and close to the $\alpha$-crystallin domain $^{86}$. Although the signaling pathways leading to serine 19 phosphorylation are unknown, the extracellular signal-regulated protein kinase (ERK) MAPK pathway appears to be responsible for phosphorylation of serine 45, and the p38 MAPK pathway is responsible for phosphorylation of serine ${ }^{87}$. Other post-translational modifications of cryAB include truncation of both the $\mathrm{N}$ terminus and the $\mathrm{C}$ terminus, deamidation, racemization, methionine oxidation, glycation, disulfide formation, addition of O-GlcNAc, and the addition of 72 mass units to the Cterminal lysine of B-crystallin ${ }^{88}$.

CryAB mutations, as well as modulation of cryAB levels have been associated with human diseases. For instance, a missense mutation in cryAB changing arginine 120 to glycine (R120G) leads to misfolded protein aggregation and is associated with cardiomyopathy and cataract formation $^{89}$. Recently, two novel mutations leading to myofibrillar myopathies (Q151X and

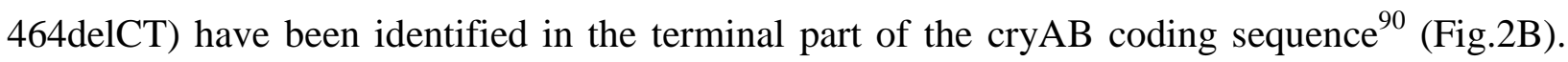
Three mutations in the cryAB gene (P20S, 464delCT and D140N) are also responsive for dominant cataract ${ }^{91}$ and two mutations $\left(\mathrm{R} 157 \mathrm{H}\right.$ and G154S) for cardiomyopathy ${ }^{91}$. Another 
mutation in the alpha B-crystallin gene (450delA) which results in the expression of an aberrant cry $\mathrm{AB}$ protein containing 184 residues in which the 35 residues at the $\mathrm{C}$-terminus are completely different from the native of protein has been shown to cause a dominant congenital posterior polar cataract in humans ${ }^{88}$ (Fig.2B).

CryAB expression is often increased in many protein conformation diseases. This upregulation can be observed in Alexander's disease, Rosenthal fibers, cortical Lewy bodies, Alzheimer disease plaques, neurofibrillary tangles as well as in synuclein deposit associated to Parkinson disease or myopathy-associated inclusion body ${ }^{92}$. The reason for the association of cryAB with these structures is potentially linked to its chaperone activity, potentially providing a first line of defense against misfolded, aggregation-prone proteins. Constitutive expression of cryAB has been detected in gliomas, prostate cancer, oral squamous cell carcinomas, renal cell carcinomas, head and neck cancer ${ }^{82}$, and increased expression of $\alpha \mathrm{B}$-crystallin was related to more advanced tumors, potentially attesting to the involvement of cryAB in regulation of cell survival and death. A high level of $\alpha \mathrm{B}$-crystallin has also been detected in basal-like breast carcinomas and preinvasive ductal carcinoma that correlated with poor clinical outcome of the patients ${ }^{82}$. Recently, a pathological role of cryAB has again been reported in breast cancer diseases, hence suggesting that it acts as an oncoprotein ${ }^{93}$. 
A

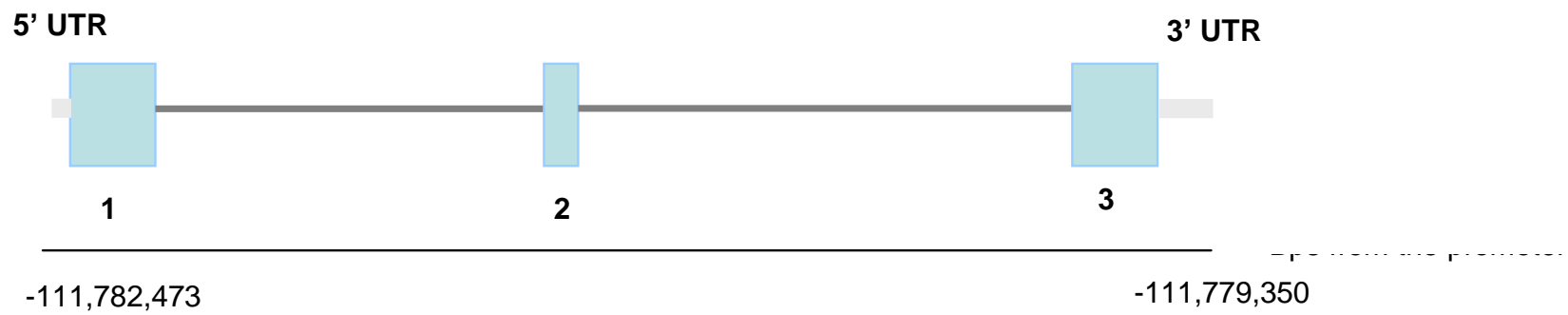

B

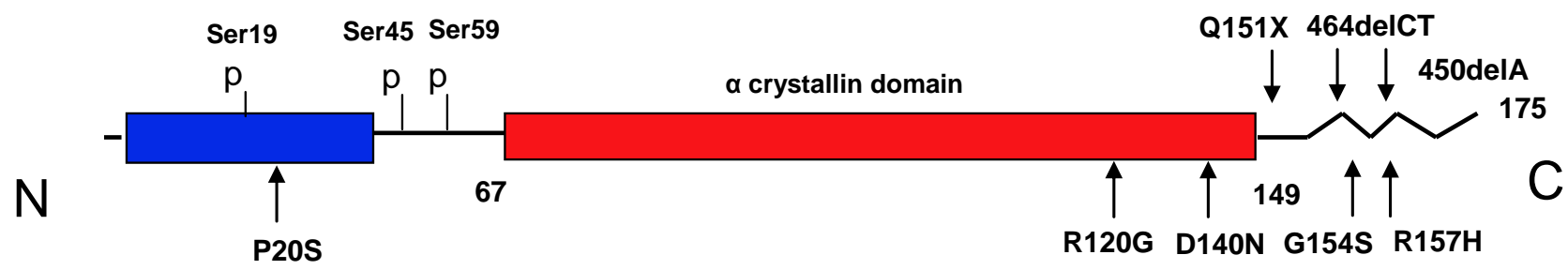

Figure 2. Organization of human cryAB genomic and protein sequence.

(A) Genomic organization of cryAB. Exons are depicted as light blue boxes, introns as grey connecting lines and the 5' and 3' untranslated regions (UTRs) are depicted as light grey boxes. The number of base pairs (Bps) are depicted on the axis. (B) Protein sequence arrangement of cryAB. Red box: $\alpha$ crystallin domain; blue box: WDPF domain; P: phosphorylated serine residues. Amino acids are indicated. Positions of point mutations that are responsible of pathologies are indicated by arrows. 464delCT: frame-shift mutant. The resulting mutant is modified from amino acid 155 and is truncated of 13 residues compared to wild type protein. 450delA: frame-shift mutant. The resulting mutant is modified from amino acid 160 to amino acid 184. This protein is larger than wild type polypeptide (175 amino acids).

\subsubsection{CryAB in the heart}

Despite initially being discovered in the lens of the eye, cryAB has been shown to be the most abundantly expressed small hsp in cardiac muscle, making up 3\% of the protein of heart homogenates ${ }^{81}$. Mutations in cryAB have been reported to lead to cardiomyopathies. A missense mutation in $\alpha \mathrm{B}$-crystallin gene, changing arginine 120 to glycine (R120G), leads to a myofibrillar myopathy associated with cardiomyopathy and cataract formation ${ }^{89}$. The cryAB R120G mutant decreases the chaperone activities of cryAB in vitro and increases protein aggregation with the intermediate filament, desmin, causing 'desmin-related cardiomyopathy'. Recently, two novel mutations leading to myofibrillar myopathies have been identified in the 
terminal part of the $\alpha \mathrm{B}$-crystallin coding sequence ${ }^{90}$. When it comes to the deletion of cryAB in cryAB KO, this mice does not lead to decreased viability ${ }^{94}$, indicating that cryAB is dispensable for survival and suggesting that other small hsps might serve redundant roles in vivo. On the other hand, these KO mice have reported increased sensitivity to $\mathrm{I} / \mathrm{R}$ injury ${ }^{95}$, suggesting that presence of cryAB is protective against stress. Furthermore, it has been shown that overexpression of cryAB in cultured rat cardiac myocytes ${ }^{87}$ or in hearts of transgenic mice ${ }^{96}$ protects from ischemia/reperfusion (I/R) damage.

\subsubsection{CryAB in apoptosis}

As described above, cryAB has been shown have protective properties. It has been demonstrated on numerous occasions that cryAB has potent anti-apoptotic properties, protecting cells from from thermal, osmotic and oxidative insult ${ }^{97}$. It has also been shown that cryAB can prevent induced apoptosis by various factors including staurosporine, TNF, okadaic acid and hydrogen peroxide $^{97}$. Moreover, cryAB negatively regulates apoptosis during myogenic differentiation ${ }^{98}$.

The protective mechanism of cryAB in response to stress has been described in part and it involves the interaction of cryAB with myofilament proteins helping to preserve contractile protein integrity and myocardial function ${ }^{99}$. Furthermore, cryAB has been shown to translocate to the mitochondria, thus reducing $\mathrm{I} / \mathrm{R}$ damage in mouse hearts during ex vivo $\mathrm{I} / \mathrm{R}^{100}$. Another component of the protective mechanism of cryAB seems to involve its phosphorylation on serine $59{ }^{101}$ in a p38-dependent manner ${ }^{102}$ in response to $\mathrm{I} / \mathrm{R}$ and other stresses. Moreover, a molecular mimic of serine 59-phosphorylated cryAB was shown to enhance the protective ability of cry $\mathrm{AB}$ against several different stresses that imitate I/R; in contrast, a cryAB mutant which blocks its phosphorylation, increased I/R-mediated myocardial cell death ${ }^{87}$.

Several steps in the apoptotic pathway are modulated by cryAB. CryAB has been shown to directly interact with the precursors of caspase 3 to suppress its activation in an immortalized rabbit lens epithelial cell line ${ }^{103}$ and in breast carcinoma cells ${ }^{104}$. In human lens epithelial cells cry $\mathrm{AB}$ has also been shown to interact with the pro-apoptotic proteins Bax and Bcl-Xs, preventing their translocation from cytosol into mitochondria, thus leading to decreased apoptosis $^{97}$. CryAB also binds pro-apoptotic protein p53, preventing its translocation to the mitochondria $^{105}$. And although the mechanism of protection against apoptosis of cryAB has been described in part, it remains to be fully elucidated in CMs. 


\subsection{Rationale of current study}

Recently, our group confirmed the anti-apoptotic properties of cryAB in a mouse model of hypertrophy, calcineurin (CNA) hypertrophy ${ }^{106}$. In this study, proteomic analysis was carried out by subjecting fractionated cardiac samples from CNA mice and their WT littermates to gel-free liquid chromatography linked to shotgun tandem mass spectrometry. We found that 290 were differentially expressed. Of the proteins whose expression was upregulated, several were associated with programmed cell death, including regulation of apoptosis ${ }^{106}$. This prompted us to look at the levels of apoptosis, and we found that in the CNA model the rates of apoptosis were minimal.

We compared the CNA proteome with the proteome from the cardiac tissue of a mouse model previously described by our group, the $\mathrm{PLN}^{\mathrm{R} 9 \mathrm{C}}$ model ${ }^{107}$. A main difference between the CNA and R9C models is that in the $\mathrm{PLN}^{\mathrm{R} 9 \mathrm{C}}$ the rates of apoptosis in cardiac tissue are significant ${ }^{106}$. The proteome comparison identified four candidates with differential expression between CNA and $\mathrm{PLN}^{\mathrm{R} 9 \mathrm{C}}$ mice, of which cryAB was significantly upregulated in CNA mice but not in the $\mathrm{PLN}^{\mathrm{R} 9 \mathrm{C}}$ mouse model. Furthermore, the viability of cultured neonatal mouse cardiomyocytes from CNA mice was higher than WTs after serum starvation, an apoptotic trigger. However following silencing of cryAB via lentivector-mediated transduction of shRNAs there was a significant reduction in neonatal cardiomyocyte viability and loss of protection against apoptosis. Given these findings and the literature available on the anti-apoptotic properties of cryAB, it was selected for further study. Specifically, I determined the mechanism by which cryAB prevents apoptosis in CMs, with a specific focus on the intrinsic pathway of apoptosis, since very few studies have focused on the anti-apoptotic mechanism of cryAB in CMs. 


\section{CHAPTER TWO: HYPOTHESIS AND AIMS}

The following aims were addressed throughout the experiments:

1. To identify the sub-cellular localization of cryAB.

2. To validate the protective effect of cryAB in CMs by knockdown by assessing the effect of cryAB levels on early and late apoptosis.

3. To identify cryAB binding interactors in the intrinsic apoptosis cascade under oxidative stress.

I hypothesized that:

1. CryAB regulation will protect $\mathrm{CMs}$ from oxidative stress.

2. CryAB modulation in $\mathrm{CMs}$ will attenuate apoptosis by binding and inhibiting caspases and/or mitochondrial proteins thus leading to decreased mitochondrial permeability. 


\section{CHAPTER THREE: MATERIALS AND METHODS}

In this section, I discuss or expand upon pertinent methods and materials that were not included in the manuscript presented in Chapter 4.

\subsection{Lentivector Production and Transduction of Neonatal Cardiomyocytes}

Lentivector compatible shRNA clones targeting mouse $\alpha$-crystallin B and $\alpha$-crystallin B cDNA were obtained from Open-Biosystems. The scrambled shRNA construct, used as a negative control was a kind gift from Dr. Stephane Angers (University of Toronto, Toronto, ON, Canada). Lentivector production and transduction of neonatal CMs were performed as described previously ${ }^{106}$. Briefly, clones were amplified using the ampicilin resistance marker in DH5- $\alpha$ cells. Plasmids were then isolated using Qiagen maxi preps according to the manufacturer's instructions. The packaging plasmid (pCMV-R8.74psPAX2, $2.5 \mu \mathrm{g}$ ), envelope plasmid (VSVG/pMD2.G, $0.3 \mu \mathrm{g})$, and the target construct plasmid ((pLKO.1, $2.7 \mu \mathrm{g})$ expressing either the shRNA or scrambled shRNA (as a negative control)) or the target construct with the cryAB cDNA( pLJM1-cryAB) were simultaneously transfected into HEK-293T cells with Optimem (Invitrogen) diluted FuGene (Roche). Neonatal cardiomyocytes were incubated with supernatant from transfected HEK-293T cells for $21 \mathrm{~h}$ after which the medium was replaced daily. Because the lentivector constructs have a puromycin resistance gene, we selected for transduced cardiomyocytes by incubating with $2 \mu \mathrm{g} / \mathrm{mL}$ puromycin for $48 \mathrm{~h}$ to kill off all nontransduced cells to ensure a homogenous population of transduced cells.

\subsubsection{Chemical Transformation of DH5- $\alpha$ cells}

The cDNA vectors were then introduced into Escherichia coli strain DH5- $\alpha$ cells by chemical transformation. Briefly, $3 \mu \mathrm{L}$ of plasmid DNA was added to $50 \mu \mathrm{L}$ of DH5- $\alpha$ cells and incubated on ice for 30 minutes and then heat shocked for 45 seconds at $42^{\circ} \mathrm{C}$. The cells were then shaken for 1 hour at $37^{\circ} \mathrm{C}$ with $950 \mu \mathrm{L}$ of SOC media (Sigma). Finally, the transformed cells were pelleted by centrifugation (10000 rpm, 1 minute), re-suspended in $150 \mu \mathrm{L}$ of SOC media, and spread onto LB 
agar plates with ampicillin resistance $(50 \mu \mathrm{g} / \mathrm{mL})$ under sterile conditions. The plates were incubated overnight at $37^{\circ} \mathrm{C}$.

\subsubsection{Amplification and Maxi Preparations of cryAB cDNA}

To amplify the cryAB cDNA, $250 \mathrm{~mL}$ of sterile 2x YT liquid culture (BioShop) with ampicillin (50 $\mu \mathrm{g} / \mathrm{mL}$ ) was inoculated with a sample of DH5- $\alpha$ cells picked from an individual colony from the LB agar plates. The cultures were shaken overnight at $37^{\circ} \mathrm{C}$. To isolate the plasmid DNA, the maxi preparation method was performed using Qiagen's Plasmid Maxi Kit. Approximately after 16-18 hours of shaking, the bacterial culture was centrifuged at $4100 \mathrm{rpm}$ for 15 minutes at $4^{\circ} \mathrm{C}$ and the supernatant discarded. The pellet was completely resuspended in $10 \mathrm{~mL}$ of cold P1 Resuspension Buffer containing RNAse, with vortexing. $10 \mathrm{~mL}$ of P2 Lysis Buffer was then added and thoroughly mixed by gently inverting the tube several times. Immediately after, the reaction was terminated with the addition of $10 \mathrm{~mL}$ of P3 Neutralization Buffer and mixing by inverting. The samples were incubated on ice for 20 minutes and then centrifuged at $4100 \mathrm{rpm}$ for 20 minutes at $4^{\circ} \mathrm{C}$. The resulting supernatant was applied to a QIAGEN-tip 500 column, which was already equilibrated by allowing $20 \mathrm{~mL}$ of Buffer QBT to drain through by gravity flow. Three successive washes were performed with $30 \mathrm{~mL}$ of Buffer QC to remove all contaminants. $15 \mathrm{~mL}$ of Buffer QF was then added to elute the bound DNA and collected in a centrifuge tube. To precipitate the DNA, $10.5 \mathrm{~mL}$ of isopropanol was added and mixed by inverting and centrifuged immediately at $12000 \mathrm{rpm}$ for 30 minutes at $4^{\circ} \mathrm{C}$. The supernatant was discarded slowly and the DNA pellet was washed by adding $20 \mathrm{~mL}$ of $70 \%$ ethanol and centrifuging at $12000 \mathrm{rpm}$ for 15

minutes at $4{ }^{\circ} \mathrm{C}$. The supernatant was again removed slowly and the pellet was allowed to air-dry to remove all traces of ethanol. Finally, the pellet was dissolved in $1 \mathrm{~mL}$ of TE Buffer and the resulting DNA concentration was determined using an Ultrospec ${ }^{\mathrm{TM}} 2100$ pro UV/Visible Spectrophotometer (GE Healthcare).

\subsection{Detection of Sub-cellular Distribution}

\subsubsection{Sub-cellular Fractionation of Adult Mouse Hearts}

Adult mice were euthanized by carbon dioxide asphyxiation. Hearts were harvested and ventricular tissue was isolated. The tissue was rinsed with ice-cold PBS to remove any remaining blood. The tissue was placed in an ice-cold lysis buffer $(250 \mathrm{mM}$ Sucrose, $50 \mathrm{mM}$ Tris-HCl $(\mathrm{pH}$ 7.4), $5 \mathrm{mM} \mathrm{MgCl}$, $1 \mathrm{mM}$ DTT, $1 \mathrm{mM} \mathrm{PMSF).} \mathrm{The} \mathrm{tissue} \mathrm{was} \mathrm{dounce-homogenized} \mathrm{and}$ 
differential centrifugation was carried out to isolate cytosolic, microsomal, and mitochondrial fractions, as described previously ${ }^{108}$. Briefly, the lysate was cleared of debris by tabletop centrifugation at $800 \mathrm{x}$ g for $15 \mathrm{~min}$. Mitochondrial and microsomal fractions were isolated from the supernatant by further centrifugation at $8000 \mathrm{x} \mathrm{g}$ and $100,000 \mathrm{x} \mathrm{g}$, respectively, and the supernatant served as the soluble cytosolic fraction. Total protein concentration calculated by using Bradford Reagent (Sigma).

\subsubsection{Sub-cellular Fractionation of Cultured Cardiomyocytes}

Cardiomyocytes from neonatal mouse were cultured as described above. These were maintained in culture for five days and on the sixth day they were either maintained in culture or stressed with $60 \mu \mathrm{M} \mathrm{H}_{2} \mathrm{O}_{2}$ for 24 hours. The CMs were then rinsed with PBS and collected in lysis buffer, as above. The CMs in lysis buffer were dounce-homogenized and differential centrifugation was carried out to isolate cytosolic and organellar (including the mitochondria) fractions, as described above.

\subsubsection{Sucrose Density Separation}

The cleared homogenized sample described above was also used for sucrose gradient separation,

as described previously ${ }^{109}$. Briefly, the supernatant was collected and layered on top of a 20-60\% linear sucrose gradient made up in $10 \mathrm{mM}$ Tris- $\mathrm{HCl}(\mathrm{pH} \mathrm{7.6)}, 10 \mathrm{mM}$ EDTA and protease inhibitors (Roche). Samples were centrifuged at 100,000 x g for $20 \mathrm{hrs}$ in a SW40Ti swinging bucket rotor. Fractions $(750 \mu \mathrm{l})$ were collected from the bottom of each gradient and total protein concentration calculated by using Bradford Reagent (Sigma).

\subsection{Immunoblot and Immunostaining Analysis}

\subsubsection{Immunoblot Detection of Sub-cellular Distribution of CryAB in Adult Whole Hearts}

The protein content from the microsomal, cytosolic, nuclear and mitochondrial fractions was harvested from WT adult mice and total protein concentration calculated by using Bradford Reagent. Also, the protein content of the fractions collected following sucrose density separation was measured. These were then subjected to standard Western blotting techniques, via SDSPolyacrylamide Gel Electrophoresis. Briefly, 10ml of resolving gel was first added and allowed to solidify 37.5:1 Acrylamide/Bis Mix (BIO-RAD) (0.2M), $1.5 \mathrm{M}$ Tris (pH 8.8) (VWR 
International) (1.5M), $12 \%$ SDS (EMD) $(0.4 \mathrm{M}), \quad 10 \%$ Ammonium Persulfate (VWR International), TEMED (EMD)]. Stacking gel was then added and solidified [H2O, 37.5:1 Acyrlamide/Bis Mix (BIO-RAD), 1.5 M Tris (pH 6.8) (VWR International), 12\% SDS (EMD), 10\% Ammonium Persulfate (VWR International), TEMED (EMD). Next, samples to be resolved were denatured by the addition of 5x Protein Loading Dye and boiling for 5 minutes before being loaded onto the gel and resolved by electrophoresis. PageRuler ${ }^{\mathrm{TM}}$ Prestained Protein Ladder (Fermentas) was used as a protein standard to gauge molecular weights. Protein concentrations were determined using Bradford assay and equal protein loading conditions were verified. Approximately $30 \mu \mathrm{g}$ of protein from each protein fraction were resolved on the gel. Proteins were then transferred from the polyacrylamide gel to a nitrocellulose membrane. The membrane was blocked in 1x PBS with 0.2\% Tween 20 (Sigma) (PBS-T) and 5\% milk for 30 minutes with shaking at room temperature. This step was followed by incubating the membrane with primary antibody diluted in 5\% milk-PBS-T solution overnight at $4 \mathrm{oC}$ on a shaker. Three 15 minutes washes with PBS-T were performed the following day, and the membrane was incubated with HRP-conjugated secondary antibody diluted in 5\% milk-PBS-T solution for 1 hour at room temperature with shaking. Subsequently three 15 minutes washes with PBS-T were performed. The blots were treated with SuperSignal West Pico Chemiluminescent Substrates (Pierce) for 5 minutes and then either imaged using Fluoro-STM Multi Imager (Bio Rad) or exposed to film in a dark room setting, which was subsequently developed. All blots were probed using commercially available antibodies: rabbit polyclonal to $\alpha$ crystallin B (cryAB; $1: 1000$ ) and serine 59-phosphorylated $\alpha$ crystallin B (Pser59, 1:1000) (Stressgen), rabbit polyclonal to caspase 3 (1:1000), and caspase 12 (Abcam; 1:1000), mouse monoclonal to caspase 9 (1:1000) and rabbit polyclonal to cytochrome C (Cell Signalling Technologies; 1:1000), goat polyclonal to Voltage Dependent Anion Channel (VDAC; 1:100), mouse monoclonal to translocase of outer mitochondrial membranes $20 \mathrm{kDa}$ (TOM 20;1:100), mouse monoclonal to cryAB (1:100) and mouse monoclonal to GAPDH (Santa Cruz Biotechnology; 1:100).

\subsubsection{Immunoblot Detection of Sub-cellular Distribution of CryAB in Stressed Neonatal Cardiomyocytes}

For detecting the translocation to the mitochondria in cultured neonatal CMs, fraction protein content was measured from control or stressed neonatal CMs with $60 \mu \mathrm{M} \mathrm{H}_{2} \mathrm{O}_{2}$ for 24 hours and the protein samples were treated and resolved as above. Following transfer to the nitrocellulose 
membrane, the membranes were probed with $\alpha$ crystallin B (cryAB; 1:1000) and serine 59phosphorylated $\alpha$ crystallin B (Pser59, 1:1000) (Stressgen), VDAC; Santa Cruz Biotechnology; 1:100) and GAPDH (Santa Cruz Biotechnology; 1:100).

\subsubsection{Immunofluorescence}

\subsubsection{Neonatal Cardiomyocyte Staining}

Lentivector transduced or wild type neonatal CMs were grown on 8-well culture slides (BDFalcon) for 5 days. After 5 days, the cells were either maintained under the same culture conditions or stressed with hydrogen peroxide. After 24 hours, the cells were washed in phosphate buffered saline (PBS) and fixed in 2\% paraformaldehyde in PBS (pH 7.0). The slides were washed 3 times with $1 \mathrm{~mL}$ of fresh permeabilization buffer $(0.2 \%$ Tween-20, $0.5 \%$ Triton $\mathrm{X}-100$ in $1 \mathrm{x}$ PBS) at $4^{\circ} \mathrm{C}$ for 15 minutes each. The washed cells were then incubated in $1 \mathrm{~mL}$ of blocking buffer (5\% FBS, 0.2\% Tween-20, 0.5\% Triton X-100 in 1x PBS) for 30 minutes at room temperature and then labelled with primary antibody diluted in blocking buffer overnight at $4^{\circ} \mathrm{C}$. The following day the slides were washed in $1 \mathrm{~mL}$ of permeabilization buffer 3 times for 15 minutes each and then incubated with fluorescent secondary antibody diluted in blocking buffer in the dark for 1 hour at room temperature. Subsequently, three 15-minute washes were performed with $1 \mathrm{~mL}$ of $1 \mathrm{x}$ PBS in the dark at room temperature, before mounting in Fluoromount ${ }^{\mathrm{TM}}$ medium (Sigma). Images were collected by using a Leica DM IRBE inverted microscope equipped with a Leica TCS SP laser scanning confocal system. Primary antibodies used for immunofluorescent analysis were obtained from collaborators or commercially: rabbit polyclonal to $\alpha$ crystallin B (cryAB; 1:1000) and $\alpha$-actinin (Santa Cruz Biotechnologies; 1:1000). Secondary antibodies used for immunofluorescent analysis were obtained commercially: Alexa 488 1:500 and Alexa 633 anti-mouse (Invitrogen) secondary antibodies 1:200, and Alexa 488 1:500 and Alexa 633 anti-rabbit (Invitrogen) secondary antibodies 1:200.

\subsubsection{Adult Cardiomyocyte Staining}

Adult CMs were isolated by placing ventricle pieces in Hank's solution with $1 \mathrm{mg} / \mathrm{mL}$ Collagenase II (Worthington Biochemicals) were subjected to gentle rocking overnight at room temperature. The next morning, the CMs were dissociated using a magnetic flea while incubated at $37{ }^{\circ} \mathrm{C}$. The suspended cells were pelleted by centrifugation at 1000 RPM for 5 minutes and washed twice in PBS. The CMs were then fixed in $90 \%$ methanol for 30 minutes at $-20^{\circ} \mathrm{C}$. The 
CMs were washed 3 times with $1 \mathrm{~mL}$ of fresh permeabilization buffer $(0.2 \%$ Tween-20, $0.5 \%$ Triton $\mathrm{X}-100$ in $1 \mathrm{x} \mathrm{PBS}$ ) at $4{ }^{\circ} \mathrm{C}$ for 15 minutes each. The washed cells were then incubated in 1 $\mathrm{mL}$ of blocking buffer (5\% FBS, $0.2 \%$ Tween-20, $0.5 \%$ Triton X-100 in 1x PBS) for 30 minutes at room temperature and then labelled with primary antibody diluted in blocking buffer overnight at $4^{\circ} \mathrm{C}$. The following day the slides were washed in $1 \mathrm{~mL}$ of permeabilization buffer 3 times for 15 minutes each and then incubated with fluorescent secondary antibody diluted in blocking buffer in the dark for 1 hour at room temperature. Subsequently, three 15-minute washes were performed with $1 \mathrm{~mL}$ of $1 \mathrm{x}$ PBS in the dark at room temperature, before mounting in Fluoromount ${ }^{\mathrm{TM}}$ medium (Sigma). Primary antibodies used for immunofluorescent analysis were obtained from collaborators or commercially: rabbit polyclonal to $\alpha$ crystallin B (cryAB; $1: 1000$ ) and serine 59-phosphorylated $\alpha$ crystallin B (Pser59, 1:1000) (Stressgen), rabbit polyclonal to caspase 3 (1:1000), and caspase 12 (Abcam; 1:1000), rabbit polyclonal to cytochrome C (Cell Signalling Technologies; 1:1000), goat polyclonal to Voltage Dependent Anion Channel (VDAC; 1:100), mouse monoclonal to translocase of outer mitochondrial membranes $20 \mathrm{kDa}$ (TOM 20; 1:100), mouse monoclonal to cryAB (1:100) and mouse monoclonal to GAPDH (Santa Cruz Biotechnology; 1:100). Secondary antibodies used for immunofluorescent analysis were obtained commercially: Alexa 488 1:500 and Alexa 633 anti-mouse (Invitrogen) secondary antibodies 1:200, and Alexa 488 1:500 and Alexa 633 anti-rabbit (Invitrogen) secondary antibodies 1:200. Images were collected by using a Leica DM IRBE inverted microscope equipped with a Leica TCS SP laser scanning confocal system.

\subsection{Viability Assays}

\subsubsection{General Viability Assay}

Neonatal CMs were subjected to $60 \mu \mathrm{M} \mathrm{H}_{2} \mathrm{O}_{2}$ for 24 hours. Viability assays were carried out using a commercially available cell counting kit (CCK-8; Dodinjo) according to the manufacturer's instructions. Briefly, a WST colorimetric substrate was added to cultured CMs and incubated at $37^{\circ} \mathrm{C}$ for 3 hours. The absorbance, which is proportional to the number of viable cells was then measured at $450 \mathrm{~nm}$ on a plate reader.

\subsubsection{Dissipation of Mitochondrial Membrane Potential Assay}

Apoptosis was determined based on dissipation of mitochondrial membrane potential, measured by JC-1 fluorescence (abcam), according to the manufacturer's instructions. Briefly, the cultured 
CMs were incubated with $20 \mu \mathrm{M} \mathrm{JC}-1$ for 10 minutes at $37{ }^{\circ} \mathrm{C}$ in the dark. Fluorescence was measured at 535-590 nm with a plate reader. MitoTracker Red CMXRos (Invitrogen) and DiOC6 $_{(3)}$ (FluoProbes) were also used for detection of respiratory cells. Briefly, the cultured CMs were incubated with $50 \mathrm{nM} \mathrm{DiOC6}(3)$ or $100 \mathrm{nM}$ MitoTracker for 30 minutes $37{ }^{\circ} \mathrm{C}$ in the dark, then washed in PBS and the fluorescence was measured with a plate reader at $488 \mathrm{~nm}$ and $657 \mathrm{~nm}$, respectively.

\subsubsection{Caspase 3 Activity Assay}

Apoptosis was also measured by detecting caspase 3 activity using a caspase 3 activity assay (R\&D Systems) as per manufacturer's instructions. Briefly, a fluorogenic substrate, DEVD-AFC, was added to lysed CMs and incubated for 2 hours. The plate was read on a plate reader at 405 nm. Caspase 3 inhibitors (R\&D Systems) were used at a concentration of $100 \mu \mathrm{M}$, according to the manufacturer's instructions.

\subsubsection{TUNEL Assay}

Lastly, apoptosis was measured by labeling of TUNEL-positive nuclei, carried out at PRPHistology laboratory, University Health Network, Toronto, ON.

\subsubsection{ROS Detection and Inhibition}

The presence of reactive oxygen species in cultured KD and WT neonatal CMs was detected using CellROX ${ }^{\mathrm{TM}}$ Deep Red reagent (Invitrogen), a fluorogenic probe according to the manufacturer's instructions. Briefly, the CMs were seeded on 96-well plates and transduced with scrambled shRNA or cryAB shRNA and maintained in culture or stressed with $60 \mu \mathrm{M} \mathrm{H}_{2} \mathrm{O}_{2}$ for 16 hours. The dye was then added to at a concentration of $5 \mu \mathrm{M}$ and fluorescence was measured with plate reader at $640 \mathrm{~nm}$. ROS scavengers, Tiron and sodium pyruvate (Sigma) were used at a concentration of $1.0 \mathrm{mM}$.

\subsection{Co-Immunoprecipitation}

Immunoprecipitations were carried out using Protein A/G-Agarose beads (Thermo Scientific). Briefly, a post-nuclear fraction was obtained from heart tissue homogenates from control hearts or hearts exposed to $100 \mu \mathrm{M} \mathrm{H}_{2} \mathrm{O}_{2}$-stressed $\mathrm{H}_{2} \mathrm{O}_{2}$. The tissue was collected in lysis buffer, as described above. The lysate was then cleared by centrifugation for $15 \mathrm{~min}$ at $2600 \mathrm{rpm}$ at $4^{\circ} \mathrm{C}$. To allow antibody-protein complex formation, the cleared lysate was incubated at $4^{\circ} \mathrm{C}$ under 
continuous rotation with either cryAB (1:100) or PcryAB (1:100) antibody in binding buffer (140 mM NaCl, 8 mM NaPO4, 2 mM KPO4, 14 mM KCl pH 7.4) and 0.1\% Triton-X100, 0.01\% BSA for 2 hours. Protein A/G-Sepharose beads were blocked in $0.1 \%$ BSA in binding buffer for 2 hours. The beads were then pelleted and added to protein sample and allowed to rotate overnight at $4^{\circ} \mathrm{C}$. Samples were washed 3 times and then eluted in $0.1 \mathrm{M}$ glycine $\mathrm{pH} 2.4$. The samples were then resolved on an SDS-PAGE gel and were probed with the following antibodies: rabbit polyclonal to $\alpha$ crystallin $\mathrm{B}$ (cryAB; 1:1000) and serine 59-phosphorylated $\alpha$ crystallin B (Pser59, 1:1000) (Stressgen), rabbit polyclonal to caspase 3 (Abcam;1:1000), and caspase 12 (Abcam; 1:1000), rabbit polyclonal to cytochrome C (Cell Signalling Technologies; 1:1000), goat polyclonal to Voltage Dependent Anion Channel (VDAC; 1:100), and mouse monoclonal to translocase of outer mitochondrial membranes $20 \mathrm{kDa}$ (TOM 20; 1:100). 


\title{
CHAPTER FOUR: $\alpha$ CRYSTALLIN B INTERACTS WITH VDAC, CASPASE 3 AND CASPASE 12 TO PREVENT APOPTOSIS FOLLOWING $\mathrm{H}_{2} \mathrm{O}_{2}$ EXPOSURE IN CARDIOMYOCYTES
}

\author{
Roxana Chis ${ }^{\mathrm{a}}$, Parveen Sharma ${ }^{\mathrm{a}}$, Nicolas Bousette, ${ }^{\mathrm{a},{ }^{*},}$ Tetsuaki Miyake ${ }^{\mathrm{a}}$, Aaron \\ Wilson $^{\mathrm{a}}$, Peter H. Backx ${ }^{\mathrm{a}, \mathrm{b}}$ and Anthony O. Gramolini ${ }^{\mathrm{a}, \mathrm{b}}$.
}

${ }^{a}$ Department of Physiology, University of Toronto; ${ }^{b}$ Heart and Stroke/Richard Lewar Centre of Excellence, University of Toronto.

*Current address: Montreal Heart Institute, University of Montreal.

Running head: $\alpha$ Crystallin B interacts with VDAC, caspase 3 and caspase 12.

Status: Am J Physiol (Heart and Circ Physiol)- (To be submitted).

\section{Author contributions}

As presented in the manuscript text: R.Chis, P.Sharma, N.Bousette, T. Miyake, A.Wilson, and A.O. Gramolini conceived and designed research. R.Chis, P. Sharma, N. Bousette, T. Miyake, and A. Wilson performed experiments. R.Chis, P.Sharma, N.Bousette, T. Miyake, and A.O. Gramolini analyzed data. R.Chis, P.Sharma, N.Bousette, T. Miyake, A.Wilson, P.H.Backx and A.O. Gramolini interpreted results of experiments. R.Chis, P.Sharma, and A.O. Gramolini prepared figures. R.Chis, P.Sharma, and A.O. Gramolini drafted manuscript. R.Chis, P.Sharma, and A.O. Gramolini edited and revised the manuscript.

Specifically, P. Sharma assisted me with sub-cellular fractionation, sucrose gradient separation, confocal microscopy and co-immunoprecipitation experiments. N. Bousette and A. Wilson performed the lentivector-mediated transductions of my cardiomyocytes. N. Bousette assisted with optimization of hydrogen peroxide doses, time-points and viability assays. N. Bousette, P. Sharma, and A.O. Gramolini helped me optimize neonatal cardiomyocyte isolation. T. Miyake provided immunofluorescent images of cryAB knock-down efficiency (appendix). I performed all remaining experimental procedures and wrote the paper with Dr Gramolini. 


\subsection{Abstract}

$\alpha$-Crystallin B (cryAB) is the most abundant small heat shock protein in cardiomyocytes (CMs), which has been shown to have potent anti-apoptotic properties. Because the mechanism by which cryAB prevents apoptosis has not been fully characterized, we examined its protective effects at the cellular level by silencing cryAB in mouse neonatal CMs using lentivector mediated transduction of shRNAs. Sub-cellular fractionation of whole hearts showed that cryAB is cytosolic under control conditions, and following $\mathrm{H}_{2} \mathrm{O}_{2}$ exposure it translocates to the mitochondria. Phosphorylated cryAB (PcryAB) is associated mainly with the mitochondria, and any residual cytosolic PcryAB translocates to mitochondria following $\mathrm{H}_{2} \mathrm{O}_{2}$ exposure. $\mathrm{H}_{2} \mathrm{O}_{2}$ exposure caused increases in cryAB and PcryAB levels and cryAB silencing resulted in increased levels of apoptosis following exposure to $\mathrm{H}_{2} \mathrm{O}_{2}$. Co-immunoprecipitation assays revealed an apparent increased level of interaction of both cryAB and PcryAB with mitochondrial VDAC, caspase 12 and caspase 3 in $\mathrm{H}_{2} \mathrm{O}_{2}$-treated hearts, compared to controls. Interactions between cry AB and TOM 20, cytochrome $\mathrm{C}$ and caspase 9 were observed, but were unchanged in $\mathrm{H}_{2} \mathrm{O}_{2}$-stressed cardiomyocytes. Our results are consistent with the conclusion that the cardio-protective effects of cryAB are mediated by its translocation from the cytosol to the mitochondria under conditions of oxidative stress and that cryAB interactions with VDAC, caspase 12 and caspase 3 may be part of its protective mechanism.

KEYWORDS: Mitochondria, reactive oxygen species, PcryAB, cardiac cell. 


\subsection{Introduction}

$\alpha$ Crystallin $\mathrm{B}$ (cryAB) is a member of the small heat shock protein family of molecular chaperones (34). It is the most abundantly expressed small heat shock protein in cardiac muscle, comprising 3-5\% of total protein in cardiac myocytes (34). Studies have demonstrated that overexpression of cry $\mathrm{AB}$ has potent cardio-protective properties in cultured rat cardiac myocytes (25) or in hearts of transgenic mice (29) protecting from ischemia/reperfusion (I/R) damage. Additionally, using in vitro models, cryAB has been shown to prevent apoptosis induced by various insults, including hydrogen peroxide $\left(\mathrm{H}_{2} \mathrm{O}_{2}\right)$ treatment (22). By contrast, silencing of cryAB increases sensitivity to $\mathrm{I} / \mathrm{R}$ injury and leads to increased cell death in mouse hearts (26). Recently, our group has shown that cryAB is protective against apoptosis in a mouse model of calcineurin (CNA) hypertrophy, which presents with elevated levels of endoplasmic reticulum (ER) stress (3).

The protective mechanism of cryAB in response to stress is not fully characterized, but previous studies have indicated that cryAB binds myofilament proteins, thereby preserving contractile protein integrity and myocardial function (2). CryAB also translocates to the mitochondria (24) and is phosphorylated on serine 59 (15) in response to ischemia and other stresses (14), which is required for limiting myocardial cell apoptosis (25), although the precise interactions mediating this protection have not been elucidated.

Apoptosis in cells occurs by two pathways, the intrinsic pathway involving mitochondria or the extrinsic pathway downstream of death receptors. The mitochondrial and death receptor pathways activate distinct apical caspases (caspase 9 or caspase 8, respectively) that activate the downstream executioner, caspase 3 (39). Ischemia or other stresses that mimic ischemia activate the intrinsic apoptotic pathway (21), leading to the opening of the mitochondrial permeability transition pore (MPTP) and further downstream apoptotic events, culminating in cellular demise (8). Global ischemia as well as reperfusion have been associated with significant increases in reactive oxygen species, including myocardial $\mathrm{H}_{2} \mathrm{O}_{2}$ content (33) which plays a significant role in oxidative stress injury (35). $\mathrm{H}_{2} \mathrm{O}_{2}$ also leads to apoptosis in CMs, by activating the intrinsic pathway of apoptosis (21), and thus make $\mathrm{H}_{2} \mathrm{O}_{2}$ a very good in vitro model of I/R injury.

The same stress stimuli that trigger apoptosis, such as oxidative stress $(9,37)$, induce the synthesis of diverse heat shock proteins that confer a protective effect against a wide range of 
cellular stresses. Recent evidence indicates that many heat shock proteins are anti-apoptotic by inhibiting one or more components in the apoptotic cascade $(1,5)$. In this regard, cryAB has been shown to interact directly with the precursors of caspase-3 to suppress its activation in an immortalized rabbit lens epithelial cell line (22) and in breast carcinoma cells (17). In human lens epithelial cells cryAB has also been shown to interact with the pro-apoptotic proteins Bax and Bcl-Xs, preventing their translocation from cytosol to mitochondria, thus leading to decreased apoptosis (23). The anti-apoptotic mechanisms of cryAB in CMs, however, have not been fully characterized. Thus, the purpose of this study was to elucidate the mechanisms by which cryAB prevents apoptosis in $\mathrm{CMs}$ with a specific focus on the mitochondrial pathway in response to $\mathrm{H}_{2} \mathrm{O}_{2}$-induced oxidative stress, an in vitro model that mimics $\mathrm{I} / \mathrm{R}$ in which $\mathrm{H}_{2} \mathrm{O}_{2}$ plays a significant role in oxidative stress injury (33). 


\subsection{Materials and Methods}

\section{Neonatal Cardiomyocyte Isolation and Culture}

Neonatal mice were euthanized using isoflurane, in accordance with procedures approved by our institutional Animal Care and Ethics Committee. Hearts were harvested and placed in icecold Hank's solution $\left(136 \mathrm{mM} \mathrm{NaCl}, 4.2 \mathrm{mM} \mathrm{KCl}, 5.6 \mathrm{mM}\right.$ dextrose, $0.44 \mathrm{mM} \mathrm{KH}{ }_{2} \mathrm{PO}_{4}, 0.34$ $\mathrm{mM} \mathrm{NaH}_{2} \mathrm{PO}_{4}, 4.2 \mathrm{mM} \mathrm{NaHCO}_{3}, 5 \mathrm{mM}$ HEPES pH 7.4, and 100 units/mL of penicillin/streptomycin (Invitrogen)). Atria were removed and discarded and ventricles were cut into small pieces (2-4/heart), and washed several times with Hank's solution. Following washes, the tissues were incubated in fresh Hank's solution with $1 \mathrm{mg} / \mathrm{mL}$ Collagenase II (Worthington Biochemicals) and subjected to gentle rocking for 2 hours at room temperature. The suspended cells were pelleted by centrifugation at 1000 rotations per minute (RPM) for 5 minutes and resuspended in $0.25 \%$ trypsin for 3 minutes. Fibroblasts were removed through pre-plating in $10 \%$ fetal bovine serum (FBS) containing media for one hour.

\section{Lentivector Production and Transduction of Neonatal Cardiomyocytes}

Lentivector compatible shRNA clones targeting mouse $\alpha$-crystallin B were obtained from Open-Biosystems (Thermo Scientific). The scrambled shRNA construct used as a negative control was a kind gift from Stephane Angers (University of Toronto, Toronto, ON, Canada). Lentivector production and transduction of neonatal CMs were performed as described previously(3). Plasmids were isolated using Qiagen Maxi preps according to the manufacturer's instructions. The packaging plasmid (pCMV-R8.74psPAX2, $2.5 \mu \mathrm{g}$ ), envelope plasmid (VSVG/pMD2.G, $0.3 \mu \mathrm{g}$ ), and the target construct plasmid (pLKO.1, $2.7 \mu \mathrm{g}$ ) expressing either the shRNA or scrambled shRNA (as a negative control) were simultaneously transduced into HEK$293 \mathrm{~T}$ cells using FuGene (Roche) diluted in Optimem (Invitrogen). Neonatal CMs were incubated with supernatant from transduced HEK-293T cells for $21 \mathrm{~h}$, after which the medium was replaced daily. Transduced CMs were selected by incubating with $2 \mu \mathrm{g} / \mathrm{mL}$ puromycin for $48 \mathrm{~h}$ to remove all non-transduced cells to ensure a homogenous population of transduced cells. 


\section{Sub-cellular Fractionation and Sucrose Gradient Separation}

Adult mice were euthanized by carbon dioxide asphyxiation. Hearts were harvested and ventricular tissue was isolated. The tissue was rinsed with ice-cold PBS to remove any remaining blood and placed in an ice-cold lysis buffer (250 mM Sucrose, $50 \mathrm{mM}$ Tris- $\mathrm{HCl}$ (pH 7.4), $5 \mathrm{mM}$ $\mathrm{MgCl}_{2}, 1 \mathrm{mM}$ DTT, $1 \mathrm{mM}$ PMSF). The tissue was dounce-homogenized and differential centrifugation was carried out to isolate nuclear, cytosolic, microsomal, and mitochondrial fractions, as described previously (6). The homogenized sample was also used for sucrose gradient separation, as described previously (31).

CMs from neonatal mice were cultured as described above. Cells were maintained in culture for five days and on the sixth day they were either maintained in culture or stressed with $\mathrm{H}_{2} \mathrm{O}_{2}$ (ranging in concentration from 0 to $200 \mu \mathrm{M}$ ) for 24 hours. The CMs were rinsed with PBS and collected in lysis buffer, as above. The CMs in lysis buffer were dounce-homogenized and differential centrifugation was carried out to isolate cytosolic and organellar (including the mitochondria) fractions, as described previously (6).

\section{Sample Preparation for Transmission Electron Microscopy (TEM)}

Cardiomyocyte fixation was performed at the Department of Pathology and Laboratory Medicine, Mount Sinai Hospital, Toronto. Briefly, neonatal CMs transduced with either scrambled shRNA (Scram shRNA) or cryAB targeting shRNA (cryAB KD) for TEM were fixed in $2 \%$ glutaraldehyde in $0.1 \mathrm{M}$ sodium cacodylate buffer, rinsed in buffer, post-fixed in $1 \%$ osmium tetroxide in buffer, dehydrated in a graded ethanol series followed by propylene oxide, and embedded in Quetol-Spurr resin. Sections of 100nm thickness were cut on an RMC MT6000 ultramicrotome, stained with uranyl acetate and lead citrate and viewed in an FEI Tecnai 20 TEM.

\section{Immunogold Labelling}

Immuno-gold labelling of cryAB was performed at the Department of Pathology and Laboratory Medicine, Mount Sinai Hospital, Toronto. Whole hearts from adult mice were fixed in $4 \%$ paraformaldehyde and $0.1 \%$ glutaraldehyde in $0.1 \mathrm{M}$ sodium cacodylate buffer, rinsed in buffer, dehydrated in a graded ethanol series with progressive lowering of temperature and 
embedded in LR White resin. Sections of 100nm thickness were cut on an RMC MT6000 ultramicrotome, labelled with 10nm gold-conjugated antibodies (anti-cryAB and anti-PcryAB), stained with uranyl acetate and lead citrate, and viewed in an FEI Tecnai 20 TEM.

\section{Viability Assays}

Neonatal CMs were subjected to $60 \mu \mathrm{M} \mathrm{H}_{2} \mathrm{O}_{2}$ for 24 hours. Viability assays were carried out using a cell counting kit (CCK-8; Dodinjo) according to the manufacturer's instructions. Early apoptosis was determined based on dissipation of mitochondrial membrane potential, measured by JC-1 fluorescent dye (Abcam), a mitochondrial dye, which was added at a concentration of $2.0 \mu \mathrm{M}$ (7) according to the manufacturer's instructions. Apoptosis was measured by detecting caspase 3 activity using an assay (R\&D Systems) as per manufacturer's instructions and by labeling of TUNEL-positive nuclei (PRP-Histology laboratory, University Health Network, Toronto, ON). The caspase 3 inhibitor, Z-D(OMe)-E(OMe)-V-D(OMe) fluoromethyl ketone (Z-DEVD-FMK; R\&D Systems), was used at a concentration of $100 \mu \mathrm{M}$ (4) according to the manufacturer's instructions.

\section{Reactive Oxygen Species (ROS) Detection and Inhibition}

The presence of ROS in cultured WT and KD neonatal CMs was detected using CellROX Deep Red reagent (Invitrogen), a fluorogenic probe, according to the manufacturer's instructions. Briefly, the CMs were seeded on 96-well plates and transduced with scrambled shRNA or shRNA for cryAB and maintained in culture or stressed with $60 \mu \mathrm{M} \mathrm{H}_{2} \mathrm{O}_{2}$. The dye was then added at a concentration of $5 \mu \mathrm{M}$ and fluorescence was measured with a Perkin Elmer plate reader. The ROS scavengers, Tiron (37), and sodium pyruvate (38) (Sigma) were used at a concentration of $1.0 \mathrm{mM}$.

\section{Co-Immunoprecipitation}

Immunoprecipitations were carried out using Protein A/G-Agarose beads (Thermo Scientific). Briefly, heart tissue homogenates were obtained from WT control hearts or hearts exposed to $100 \mu \mathrm{M} \mathrm{H}_{2} \mathrm{O}_{2}$ for 1 hour (22). The tissue was collected in lysis buffer, as described above. The lysate was cleared by centrifugation for $15 \mathrm{~min}$ at $2600 \mathrm{rpm}$ at $4^{\circ} \mathrm{C}$. To allow antibody-protein complex formation, the cleared lysate was incubated at $4{ }^{\circ} \mathrm{C}$ under continuous rotation with antibody in binding buffer $(140 \mathrm{mM} \mathrm{NaCl}, 14 \mathrm{mM} \mathrm{KCl} \mathrm{pH} 7.4)$ and $0.1 \%$ Triton- 
X100, $0.01 \%$ BSA for 2 hours. Protein A/G-agarose beads were blocked in $0.1 \%$ BSA in binding buffer for 2 hours. The beads were pelleted and added to protein sample and rotated overnight at $4^{\circ} \mathrm{C}$. Samples were washed 3 times and eluted in $0.1 \mathrm{M}$ glycine $\mathrm{pH} 2.4$.

\section{Immunoblot Analysis}

Total cellular protein was harvested from control or stressed with $100 \mu \mathrm{M} \mathrm{H}_{2} \mathrm{O}_{2}$ for 1 hour cardiac ventricular homogenates from WT animals and was subjected to standard immunoblotting. Protein concentrations were determined by Bradford assay and equal protein levels were loaded. Following antibodies were used to target specific proteins: rabbit polyclonal to $\alpha$ crystallin B (Stressgen; 1:1000) and serine 59-phosphorylated $\alpha$ crystallin B (Stressgen; 1:1000), rabbit polyclonal to caspase 3 (Abcam; 1:1000), rabbit polyclonal to caspase 12 (Abcam; 1:1000), mouse monoclonal to caspase 9 (Cell Signalling Technologies; 1:1000) and rabbit polyclonal to cytochrome C (Cell Signalling Technologies; 1:1000), goat polyclonal to Voltage Dependent Anion Channel (VDAC; Santa Cruz Biotechnology; 1:100), mouse monoclonal to translocase of outer mitochondrial membranes 20 kDa (TOM 20; Santa Cruz Biotechnology; 1:1000), and mouse monoclonal to cryAB (Santa Cruz Biotechnology ; 1:100).

\section{Immunofluorescence}

Adult CMs were isolated as described previously (30) and fixed in ice-cold $90 \%$

methanol. Staining was performed as described previously (31). A Leica DM IRBE inverted microscope equipped with a Leica TCS SP laser scanning confocal system collected the images.

\section{Statistical Analysis}

Statistical differences were determined by an analysis of variance (ANOVA) and unpaired Student's t-test. Post-hoc Tukey tests were performed when analysis of variance was significant. Results were considered significant at $\mathrm{p}<0.05$. 


\subsection{Results}

\section{The expression pattern of cryAB in mouse cardiac muscle}

CryAB protein is present diffusely in the cytosol under normal physiological conditions in CMs (20). To determine the endogenous distribution of cryAB protein among different cellular compartments, sub-cellular fractionation of adult mouse hearts was performed by differential centrifugation and sucrose gradient separation. Differential centrifugation was used to generate nuclear, organellar (including the mitochondria), microsomal, and cytosolic fractions. Positive fraction markers used were GAPDH for the cytosol, histone $\mathrm{H} 3$ for the nucleus, Na/K ATPase for the microsomes and VDAC for the organellar/mitochondrial fraction. As observed in Figure 1A, the fractions were relatively pure as GAPDH was found exclusively in the cytosolic fraction, VDAC was found restricted to the mitochondrial fraction, Histone H3 was found preferentially in the nuclear fraction, and $\mathrm{Na} / \mathrm{K}$ ATPase was found predominately in the microsomal fraction with some expression in the organellar/mitochondrial fraction. Immunoblot analysis for the expression of cryAB in each sub-cellular fractions showed the highest levels ( $83 \%$ of total levels) seen in the cytosol, in agreement with the literature (20). Remaining levels were: $8 \%$ of total levels in the microsomal fraction, $8 \%$ in the nuclear fraction, and negligible levels $(<1 \%)$ in the organellar fraction (Fig. 1A). The cytosolic localization of cryAB was further confirmed by sucrose gradient separation of whole heart lysates. Figure $1 \mathrm{~B}$ shows that cryAB was restricted to fractions 4, 5 and 6. This was consistent with GAPDH, a known cytosolic protein, which was also detected in fractions 4, 5, and 6. Protein disulfide isomerase (PDI), an ER marker, was isolated within the heavy fractions, corresponding to mainly fraction 1 and showed some expression in fractions 2, 3 and 4. Conversely, the Na/K ATPase, a plasma membrane protein found normally within the microsome biochemical fraction (19), was isolated within the light fractions (with some expression in fractions 8, 9, 10, 11 and 12). Finally, electron microscopy images depict the distribution of cryAB in the cytosol and PcryAB predominantly in the mitochondria in adult mouse cardiac muscle. Representative images in Figure 1C-F show that $\sim 75 \%$ of gold-labelled cryAB (111 of 149 particles in ten separate images) were found in the cytosol, $11 \%$ in the mitochondria, and $14 \%$ associated with the sarcomeres. When similar experiments were performed using gold-labelled PcryAB antibody, $75 \%$ of the gold particles 
were found in the mitochondria, while $10 \%$ were found in the cytosol, and $15 \%$ were in the sarcomeres (73 total particles were observed in ten separate images).

\section{CryAB silencing induces loss of viability in CMs}

To determine whether cryAB expression in mouse CMs affects cell viability, we silenced cry $\mathrm{AB}$ using lentivector mediated transduction of shRNA targeting cryAB expression. Figure $2 \mathrm{~A}$ shows the reduced levels of cryAB expression following transduction of cryAB-specific shRNA virus (cyAB KD) relative to a 'scrambled' shRNA control vector (Scram shRNA). The transduction resulted in reduced cryAB expression to $28 \pm 2 \%$ in the $\mathrm{KD} \mathrm{CM}$ compared to Scram shRNA CMs (Fig. 2A; p < 0.05).

Since cryAB has anti-apoptotic properties (22 26, 30) and previous studies have illustrated that it prevents apoptosis induced by oxidative insult (22), the neonatal CMs transduced with either the cryAB targeting lentivectors or the scrambled shRNA control lentivectors, were analyzed following administration of $60 \mu \mathrm{M}, 100 \mu \mathrm{M}$ or $200 \mu \mathrm{M} \mathrm{H}_{2} \mathrm{O}_{2}$ for 24 hours (37) to explore the role of cryAB on the viability of CMs exposed to $\mathrm{H}_{2} \mathrm{O}_{2}$ oxidative stress. CryAB KD neonatal CMs showed a significant reduction in viability compared with the neonatal CMs transduced with the scrambled control construct (Scram shRNA) at all $\mathrm{H}_{2} \mathrm{O}_{2}$ concentrations used (Fig. 2B). At $0 \mu \mathrm{M} \mathrm{H}_{2} \mathrm{O}_{2}$, the viability in the cryAB KD CMs was $50 \pm 6 \%$ compared to the levels seen in the Scram shRNA CMs $(\mathrm{p}<0.05)$. At $60 \mu \mathrm{M}$, the scram shRNA CMs had a viability of $73 \pm 4 \%$ compared to reference levels, while the cryAB KD CMs exhibited a viability of only $29 \pm 2 \%$ (p < 0.05). At $100 \mu \mathrm{M} \mathrm{H}_{2} \mathrm{O}_{2}$, scrambled-infected cells showed a reduced viability to $64 \pm 2 \%$ of the pre-treated values, however, in the cryAB KD CMs there was a significantly lower viability of only $16 \pm 5 \%(\mathrm{p}<0.05)$. When cells were treated with $200 \mu \mathrm{M} \mathrm{H}_{2} \mathrm{O}_{2}$, there was a slightly lower level of viability in scram shRNA CMs down to $47 \pm$ $7 \%$; and the cryAB KD CMs remained low with only $20 \pm 2 \%$ survival rates $(\mathrm{p}<0.05$ ). Altogether, cryAB KD appears to promote cell death at baseline and following exposure to $\mathrm{H}_{2} \mathrm{O}_{2}$-induced oxidative stress and exposure to $\mathrm{H}_{2} \mathrm{O}_{2}$ seems to cause reductions in viability in scrambled and cryAB KD CMs, which are more pronounced in the KD CMs. Since cryAB KD alone induced significant cell death and treatment with $60 \mu \mathrm{M} \mathrm{H}_{2} \mathrm{O}_{2}$ lead to significant reductions in viability in both scrambled and cryAB $\mathrm{KD}$ CMs, subsequent experiments were performed using $60 \mu \mathrm{M} \mathrm{H}_{2} \mathrm{O}_{2}$ to exacerbate the cell death initially observed at baseline in KD CMs. 
To confirm activation of apoptosis at baseline and following exposure to $\mathrm{H}_{2} \mathrm{O}_{2}$ we measured caspase 3 activity. Activity was determined in control and cryAB KD CMs under resting conditions and following exposure to $\mathrm{H}_{2} \mathrm{O}_{2}$, in the absence or presence of caspase 3 inhibitors (Fig.2C). Caspase 3 activity levels in the scrambled CMs under baseline conditions were set as a reference level of 1 arbitrary unit (A.U.). In the cryAB KD CMs, caspase 3 activity levels were significantly higher than in Scram controls $(130 \pm 2 \%)$ at rest $(\mathrm{p}<0.05)$. The addition of caspase inhibitors had negligible effects on caspase 3 activity levels in the Scram CMs under basal conditions, bringing the levels down to $90 \pm 2 \%$ compared to baseline conditions. In the KD CMs, however, caspase 3 activity levels were significantly lowered to $70 \pm$ $2 \%$ of their baseline levels following the addition of caspase 3 inhibitors $(\mathrm{p}<0.05)$. As expected, following exposure to $\mathrm{H}_{2} \mathrm{O}_{2}$, caspase 3 activity levels increased to $240 \pm 4 \%$ in the control CMs ( $<$ 0.05), while in the KD CMs they increased $\sim$-fold with addition of $\mathrm{H}_{2} \mathrm{O}_{2}(460$ $\pm 5 \%$ relative to Scram CMs baseline levels). The addition of $\mathrm{H}_{2} \mathrm{O}_{2}$ together with caspase 3 inhibitors had caspase 3 activity levels close to the levels observed at basal conditions $(110 \pm$ $3 \%)$ in the Scram shRNA CMs and the KD CMs (120 $\pm 3 \%$ of reference levels). We confirmed the levels of apoptosis in Scram shRNA CMs and cryAB KD CMs at baseline and following exposure to $60 \mu \mathrm{M} \mathrm{H}_{2} \mathrm{O}_{2}$ by TUNEL-staining. Analysis of TUNEL-positive nuclei (Fig. 2D) shows a significantly higher percentage of TUNEL-positive nuclei in the cryAB KD CMs compared to Scram shRNA CMs at baseline $(30 \pm 3 \%$ vs. $9 \pm 2 \%$; p <0.05) and following exposure to $\mathrm{H}_{2} \mathrm{O}_{2}(67 \pm 6 \%$ vs. $30 \pm 5 \%$, $\mathrm{p}<0.05)$. These results suggest that the KD CMs were undergoing apoptosis by activation of caspase 3 more readily when compared to Scram CMs at baseline, an effect that was exacerbated following exposure to $\mathrm{H}_{2} \mathrm{O}_{2}$, suggestive of a protective role for cryAB against apoptosis.

To determine whether cryAB knockdown and exposure to $\mathrm{H}_{2} \mathrm{O}_{2}$ are interacting variables affecting CM viability, we performed a two-way analysis of variance and found that, while cryAB KD has significant effects on lowering viability (\# at $\mathrm{p}<0.05$ ) and exposure to $\mathrm{H}_{2} \mathrm{O}_{2}$ also lowers viability significantly at all concentrations tested in scrambled and cryAB KD CMs (* and ** at $\mathrm{p}<0.05$ ), the effects of cryAB $\mathrm{KD}$ and $\mathrm{H}_{2} \mathrm{O}_{2}$ exposure combined on CMs viability seem to be additive. In other words, the interaction of cryAB KD and $\mathrm{H}_{2} \mathrm{O}_{2}$ exposure only approaches statistical significance $(\mathrm{p}=0.1)$. However, in Fig. $2 \mathrm{~A}$ we show that cryAB silencing 
was not $100 \%$ efficient, which could account for the higher than expected viability that was observed in cryAB KD CMs in the presence of extrinsic oxidative stress.

\section{Upregulation and translocation of cry $A B$ to the mitochondria under stress conditions}

The expression levels of cryAB have been shown to increase in response to $\mathrm{H}_{2} \mathrm{O}_{2}$ exposure (32). To determine whether similar upregulation occurs in our CM model, cardiomyocytes isolated from adult mice were treated with $100 \mu \mathrm{M} \mathrm{H}_{2} \mathrm{O}_{2}$, and cryAB levels were determined. In comparison to untreated conditions, following exposure to $\mathrm{H}_{2} \mathrm{O}_{2}$ total cryAB levels were significantly increased (1.8-fold over control levels; $\mathrm{p}<0.05)$. PcryAB levels were also increased significantly under $\mathrm{H}_{2} \mathrm{O}_{2}$ treatment with a 2-fold increase $(\mathrm{p}<0.05)$ in protein levels (Fig. 3A).

Under conditions of $\mathrm{I} / \mathrm{R}$, cryAB translocates to the mitochondria and contractile units (16). Following $I / R$, phosphorylation of cryAB on the serine-59 residue enhances protection against apoptosis and it has been stipulated that cryAB and PcryAB may have different protective effects in CMs (16). To determine the distribution of cryAB and PcryAB in $\mathrm{H}_{2} \mathrm{O}_{2}$ induced oxidative stress, sub-cellular fractionation of cultured control neonatal mouse CMs, and CMs exposed to $60 \mu \mathrm{M} \mathrm{H}_{2} \mathrm{O}_{2}$ for 24 hours was performed. Figure $3 \mathrm{~B}$ and $\mathrm{C}$ show higher levels of cry $\mathrm{AB}$ expression in the cytosol and lower levels in the mitochondria. Under normal culture conditions, a 2:1 cytosolic to mitochondrial ratio distribution of cryAB was observed. In contrast, upon exposure to $60 \mu \mathrm{M} \mathrm{H}_{2} \mathrm{O}_{2}$, cryAB translocates to the mitochondria resulting in 1:5 cytosolic to mitochondrial ratio distribution $(\mathrm{p}<0.05)$. PcryAB, on the other hand, was found at very low levels in the cytosol compared to the levels observed in mitochondria. Expression ratio levels of PcryAB were calculated to be 1:5 cytosolic to mitochondrial ratio distribution. Under stress conditions PcryAB was almost exclusively associated with the mitochondria, with only less than $5 \pm 0.6 \%$ of total PcryAB detected in the cytosol ( $\mathrm{p}<0.05$; Fig. 3B-C). GAPDH and VDAC were used as fraction markers for the cytosol and mitochondria, respectively. Under control conditions, GAPDH and VDAC were found exclusively in the cytosolic and mitochondrial fraction, respectively. Taken together, these findings suggest that part of the protective mechanism of cryAB following exposure to oxidative stress may involve its translocation to the mitochondria. 


\section{CryAB silencing induces mitochondrial dysfunction}

Since CM viability was decreased significantly under control conditions in cryAB KD CMs, it was of interest to determine the cause for the increased cell death in the absence of a stressor. The association of cryAB and PcryAB with the mitochondria following exposure to oxidative stress suggested potential mitochondrial involvement in cryAB knockdown-induced apoptosis. Furthermore, previously, cryAB R120G transgenic mice were demonstrated to exhibit desmin-related cardiomyopathy where mitochondrial respiration was compromised leading to alterations in the permeability transition pore, compromised inner membrane potential and elevated levels of apoptosis (21). To test whether reduced levels of cryAB might affect the mitochondria by induction of oxidative stress, the levels of reactive oxygen species (ROS) in cryAB KD and scrambled CMs were determined at baseline, following exposure to $60 \mu \mathrm{M} \mathrm{H}_{2} \mathrm{O}_{2}$ and in the presence of ROS scavengers (Fig. 4B). At baseline, ROS levels in the cryAB KD CMs were 1.9-fold higher than the levels observed in the scrambled CMs (\# at $\mathrm{p}<0.05$ ). With the addition of ROS scavengers, ROS levels were decreased in the scrambled CMs to $75 \pm 2 \%$ of the levels observed in scrambled-infected cells without ROS inhibition, cryAB-KD CMs treated with the ROS scavengers, ROS levels were decreased to near control levels of $110 \pm 3 \%$ relative to baseline levels observed in scrambled CMs (significantly different than untreated cryAB-KD CMs; $\mathrm{p}<0.05)$. These results suggest that some oxidative stress was associated with the culture process, but that there is higher production of ROS by the mitochondria in the cryAB KD CMs with or without scavengers.

Following the addition of $\mathrm{H}_{2} \mathrm{O}_{2}$ there was a 2.4-fold increase in ROS levels in the scrambled CMs $(\mathrm{p}<0.05)$ and much greater 5.8-fold increase in the cryAB KD CMs $(\mathrm{p}<0.05)$ relative to reference levels in scrambled CMs. In the presence of $\mathrm{H}_{2} \mathrm{O}_{2}$ and ROS scavengers, cells with scrambled shRNA showed a $54 \pm 7 \%$, decrease in ROS levels compared to $\mathrm{H}_{2} \mathrm{O}_{2}$ treatment alone, while in KD CMs ROS levels were decreased to $55 \pm 4 \%$ compared of the levels observed with $\mathrm{H}_{2} \mathrm{O}_{2}$ treatment alone, but still remained elevated compared to the Scram shRNA CMs ( $\mathrm{p}<$ 0.05, Fig. 4B). These results suggest that the oxidative stress observed at baseline in the Scram shRNA and KD CMs is exacerbated by the addition of exogenous $\mathrm{H}_{2} \mathrm{O}_{2}$.

To determine the effect of reduced expression of cryAB and ROS production on mitochondrial function, we assessed dissipation of mitochondrial membrane potential, an 
indicator of early apoptosis, by using the mitochondrial dye, JC-1. Increased dissipation of the mitochondrial membrane potential in cryAB $\mathrm{KD} \mathrm{CMs}$ was observed compared to scrambled/control CMs at baseline. This effect was illustrated by a significant loss of JC-1 red fluorescence in cryAB KD CMs to $63 \pm 8 \%$ of the levels that were observed in scrambled/control CMs at baseline $(\mathrm{p}<0.05)$. In the presence of ROS scavengers under control conditions, JC-1 fluorescence was increased by $12 \pm 7 \%$ in control CMs relative to initial levels at rest $(\mathrm{p}<0.05)$ and by $2 \pm 0.5 \%$ in the KD CMs $(\mathrm{p}<0.05)$ relative to their initial levels at rest. These results suggest that both control and KD CMs were exposed to oxidative stress under cell culturing conditions, but that dissipation of membrane potential only occurred in the KD CMs in the absence or presence of ROS scavengers. The increased ROS observed in the KD CMs may occur alongside mitochondrial structure alterations that cannot be salvaged by the removal of ROS alone, pointing to an involvement of cryAB at the mitochondrial level. Following exposure to 60 $\mu \mathrm{M} \mathrm{H}_{2} \mathrm{O}_{2}$, JC-1 fluorescence decreased to $32 \pm 2 \%$ in Scram shRNA CMs $(\mathrm{p}<0.05)$ and to only $9 \pm 1 \%(\mathrm{p}<0.05)$ in the cryAB KD CMs (Fig. 4A). In the presence of $\mathrm{H}_{2} \mathrm{O}_{2}$, together with $\mathrm{ROS}$ scavengers, JC-1 fluorescence was $63 \pm 3 \%$ of its original value in control CMs. In KD CMs, the fluorescence was $26 \pm 3 \%$ of its original value $(\mathrm{p}<0.05)$. Together, these results suggest that the increased ROS levels in the cryAB KD CMs contribute to the loss of mitochondrial membrane potential, which cannot be overcome with free radical scavengers (Fig. 4C).

Since we observed mitochondrial dysfunction and altered respiration in the cryAB KD CMs, we investigated whether mitochondrial ultrastructure was disturbed by cryAB silencing. Electron microscopy images showed that in the CMs transduced with the scrambled shRNA, the mitochondria were intact, with dense, well-ordered cristae (Fig. 5A-B). However, in the cryAB KD CMs, approximately half of the observed mitochondria exhibited altered morphology, and loss of density and organization of cristae, suggestive of mitochondrial swelling and rupture of the inner membrane (Fig. 5C-D). These results are consistent with our earlier results of mitochondrial dysfunction and increased ROS production (Fig. 4B-C). 


\section{Identification of cryAB interactions with proteins involved in apoptosis following exposure to $\mathrm{H}_{2} \mathrm{O}_{2}$}

Our results suggest an involvement of cryAB in preventing ROS-induced apoptosis at the level of the mitochondria. Furthermore, in response to oxidative stress, cryAB and PcryAB have been observed to associate with the mitochondria (16), thus an interaction with mitochondrial proteins (such as VDAC and TOM 20) may prevent the mitochondrial permeability pore from forming and, ultimately, prevent apoptosis. We hypothesized that cryAB potentially also regulates the apoptotic cascade downstream of the mitochondria via interacting with cytochrome C, caspase 9, caspase 12 and caspase 3. To mimic and exacerbate further the oxidative stress observed following cryAB knockdown, we exposed adult WT CMs to treatment with $100 \mu \mathrm{M}$ $\mathrm{H}_{2} \mathrm{O}_{2}$. A co-localization of cryAB with any of these proteins shown by qualitative fluorescence images of adult $\mathrm{CMs}$ exposed to $100 \mu \mathrm{M} \mathrm{H}_{2} \mathrm{O}_{2}$ stained with cryAB, in green, and TOM 20, VDAC, cytochrome $\mathrm{C}$ or caspase 3, in red (Fig. 5A). The far right panel depicts co-localization of cryAB with TOM 20, VDAC, cytochrome $\mathrm{C}$ and caspase 3, in yellow, suggestive of a potential interaction of cryAB with these proteins following exposure to $\mathrm{H}_{2} \mathrm{O} 2$ (Fig. 5A). To determine whether cry $A B$ interacts with any of these proteins, we immunoprecipitated cry $A B$ and PcryAB, under control conditions and following exposure to $100 \mu \mathrm{M} \mathrm{H}_{2} \mathrm{O}_{2}$. CryAB and PcryAB both were found to coprecipitate with mitochondrial protein TOM 20 and with caspase 9. CryAB and PcryAB formed complexes with TOM 20, which were increased 1.2-fold and 1.4fold, respectively, under stressed conditions compared to control levels; and increased 1.4-fold and 1.5-fold with caspase 9. CryAB. PcryAB was found to precipitate with cytochrome $\mathrm{C}$ under both control and stress conditions at a 1:1 ratio (Fig. 5A). Interestingly, cryAB precipitated VDAC, and for this interaction, there was a significantly higher level of VDAC precipitated under stress conditions when compared with control of cryAB with VDAC $(2877 \pm 165$ A.U. in control cells compared to $8761 \pm 429$ A.U. in stressed cells; * at p < 0.05). PcryAB also interactions with VDAC, and amount of interacting VDAC with PcryAB increased under stress conditions, $549 \pm 26$ A.U. vs $2452 \pm 1015$ A.U. (p < 0.05). Caspase 12 was found in the cryAB precipitate and greater band intensities were seen under stress conditions (3684 \pm 247 A.U. vs $13838 \pm 537$ A.U.; p < 0.05). Uncleaved Caspase 3 was seen in the cryAB precipitate although it did not change significantly between conditions (19702 \pm 402 in control vs $31523 \pm 1598$ under stress; $p>0.05)$. Similar results were obtained in PcryAB precipitations as caspase 3 formed 
immune-complexes with PcryAB increased from $24263 \pm 692$ A.U. under control conditions to $44274 \pm 1067$ A.U. under stress conditions, but this did not reach statistical significance (p > 0.05). The interaction of PcryAB with cleaved caspase 3 also seemed to increase following exposure to hydrogen peroxide when compared to control conditions (2432 \pm 311 A.U. from $2002 \pm 434$ A.U., or a 1.2-fold increase, but this was not statistically significant) (Fig. 5B-C). However, we did observe a 2.2-fold increase in the interaction of cryAB with cleaved caspase 3 $(2375 \pm 460$ under control conditions and $5225 \pm 255$ A.U. in stressed cells $(\mathrm{p}<0.05))$. We made numerous attempts to determine interactions of either cryAB or PcryAB with Bcl-Xs and Bax (23), but were unable to detect signals under these conditions. 


\subsection{Discussion}

This study provides evidence of a cytosolic to mitochondrial translocation of cryAB and PcryAB in adult mouse $\mathrm{CMs}$ in response to $\mathrm{H}_{2} \mathrm{O}_{2}$-induced oxidative stress. Upregulation of total cryAB levels following $\mathrm{H}_{2} \mathrm{O}_{2}$ exposure and the significant reduction in viability in mouse neonatal cryAB $\mathrm{KD} \mathrm{CMs}$ suggest that cryAB is protective against apoptosis. Immunoprecipitation assays indicated that cry $\mathrm{AB}$ intervenes at multiple points in the intrinsic apoptotic cascade by interacting with VDAC, caspase 3 and caspase 12 (activated by ER stress (27)) and that these interactions may be part of the protective mechanism of cryAB in CMs.

One of the conclusions of this study is that the protective mechanism of cryAB in ROSinduced cell death may involve its stabilization of the mitochondria. Our results extend earlier findings regarding cryAB localization (25). PcryAB is associated with the mitochondria even under control conditions (Fig. 1E-F) and this association increases significantly under conditions of oxidative stress (Fig. 2). It is possible that through this association PcryAB binds and stabilizes or modulates the activity of MPTP proteins, preventing pore opening and ensuing apoptosis. Furthermore, our results indicate that the interaction of cryAB or PcryAB with VDAC may be important for the protective mechanism, as it is significantly increased under stress conditions (Fig. 5). VDAC is one of the proteins that make up the MPTP, the opening of which induces apoptosis (8). By interacting with VDAC, cryAB and/or PcryAB may stabilize the mitochondrial membrane, preventing the opening of the pore and thus blocking the first step in the mitochondrial pathway of apoptosis. In agreement with this, studies have shown that MPTP is activated by unfolding of pore proteins upon stress (12), which could act as a stimulus drawing chaperones such as cryAB to the mitochondria following exposure to stress. We have shown by electron microscopy that PcryAB is present in the mitochondria; thus, interaction with proteins found in the inner mitochondrial membrane would be feasible. Furthermore, studies have shown that cytosolic heat shock proteins can localize to the interior of the mitochondria- for instance, Hsp25 binds to and protects mitochondria cytochrome Complex I from oxidative stress in PC12 cells (5). Therefore, the interaction of cryAB with proteins in the inner mitochondrial membrane may be a central mechanism by which cryAB prevents apoptosis. Interestingly, cryAB has been shown to interact with pro-apoptotic proteins Bax and Bcl-Xs, preventing their translocation from cytosol into mitochondria, thus maintaining the mitochondrial integrity and leading to decreased apoptosis (23). 
A second conclusion to be drawn from the present study is that cryAB is protective against oxidative stress-induced apoptosis in CMs at baseline and following extrinsically exacerbated oxidative stress. Much like other heat shock proteins that confer a protective effect against a wide range of cellular stresses, cryAB is induced by cellular stresses (9) such as oxidative stress and is involved in protection against stress. We found that at baseline, cryAB KD CMs are already subject to oxidative stress by increased ROS production that decreases their viability. Previously, it was shown that in a heat shock factor 1 (hsf1) knock out mouse, which has reduced expression of hsp70, hsp25 and cryAB, there is increased mitochondrial oxidative damage (40) and here we show that silencing of cryAB alone induced increased oxidative damage at the mitochondrial level as well as loss of mitochondrial membrane potential. The mitochondria are a major source of ROS and oxidative stress, and they trigger ROS-induced activation of the intrinsic apoptotic pathway by releasing cytochrome $C$ (9). Hence, KD of cryAB could contribute to increased mitochondria-induced apoptosis by ROS-induced ROS production (41). Increased ROS production as a result of alterations to the mitochondrial membrane potential and electron transport chain would trigger subsequent ROS production, leading to apoptosis. Electron microscopy images in this study support this speculation, as they show significantly altered mitochondrial ultrastructure in cryAB-silenced cardiomyocytes, suggestive of mitochondrial permeabilization and rupture (Fig. 5), which would lead to apoptosis induction. Furthermore, given the ability of antioxidants to protect against apoptosis in I/R injury by blocking the increased expression of p53, Bax, and caspase-3, and inhibiting caspase-3 activation (28), it may be that the oxidative stress imposed by cryAB KD functions by the opposite mechanism, increasing the expression of pro-apoptotic proteins and stimulating apoptosis.

Downstream of the interactions with mitochondrial proteins, cryAB may also protect from apoptosis induced by oxidative stress by interacting with caspase 3 and caspase 12, further suggesting an involvement of cryAB in protection against not only the intrinsic pathway but also the ER stress-induced apoptosis. Caspase 3 is activated in response to exposure to $\mathrm{H}_{2} \mathrm{O}_{2}$, which induces the intrinsic pathway of apoptosis (37). In our study and others (17, 18, 20), cytosolic cryAB appears to interact with the uncleaved caspase 3 to prevent its cleavage, therefore, its activation, thus preventing progression along the apoptotic cascade. In the absence of cryAB, caspase 3 activity increases. It results in higher levels of apoptosis observed in the cryAB KD 
CMs. The increased apparent interactions of cryAB with cleaved caspase 3 observed in the present study which has not been reported previously, to our knowledge. This may be indicative of the potential ability of cryAB to bind and inhibit active caspase 3, even following its cleavage, thus potentially inhibiting its proteolytic activity and cleavage of substrates in the cell and providing an additional point for intervention in preventing apoptosis in CMs. CryAB but not PcryAB interaction with caspase 12 is significantly different under stress when compared to control conditions. Caspase 12 is activated by ER stress (27), suggesting that cytosolic cryAB is also potentially involved in preventing ER stress-induced apoptosis. ER stress also contributes to apoptosis, by leading to the release of $\mathrm{Ca}^{2+}$ from the ER causing depletion of ER calcium and activating $\mathrm{Ca}^{2+}$-dependent endonucleases involved in DNA fragmentation (10). Furthermore, $\mathrm{Ca}^{2+}$ is also believed to modulate cytochrome $\mathrm{C}$ release directly by regulating the MPTP, leading to apoptosis (11). As a molecular chaperone, cryAB would be induced in response to ER stress, suggesting that in cryAB-silenced cells ER stress would lead to rampant apoptosis. This comes in agreement with our previous findings that show an upregulation of cryAB in response to ER stress, as well as decreased viability of cardiomyocytes in the absence of cryAB under conditions of augmented ER stress (3). This study does not differentiate between apoptosis by the intrinsic pathway and apoptosis induced by ER stress, but determining the contribution of ER stress to apoptosis in the absence of cryAB would be of interest.

Regulating the levels of apoptosis is an attractive target for ameliorating any ischemic injury in the heart. Our results contribute to delineating the involvement of cryAB in the intrinsic and ER stress pathway of apoptosis activated by ROS-induced oxidative damage. ROS are known to play a major role in the pathogenesis of myocardial dysfunction in a variety of conditions including I/R injury (13), with $\mathrm{H}_{2} \mathrm{O}_{2}$, playing a significant role in oxidative stress injury (35). Therefore, determining where in the apoptotic cascade cryAB intervenes to prevent $\mathrm{H}_{2} \mathrm{O}_{2}$ injury can have therapeutic implications for cardiac disease. For instance, it has been shown that administration of exogenous cryAB significantly improves murine cardiac function following I/R injury, and that it decreases caspase 3 activity and apoptosis in hypoxic human endothelial cells, but not in mouse atrial cardiomyocytes (36). Our study determined the antiapoptotic response and mechanism of cryAB in ventricular CMs, a different CM type than the one tested in the Velotta et al. study and shows that cryAB intervenes and modulates apoptosis by interacting with VDAC, caspase 3 and caspase 12 in ventricular CMs. This suggests that 
therapeutic administration of cryAB could target and inhibit the intrinsic and ER stress pathways of apoptosis in ventricular CMs, thus potentially contributing to improved cardiac function. Further studies focusing on the extrinsic pathway of apoptosis and alternate death pathways, like autophagy and necrosis, would therefore prove quite insightful. 


\subsection{Disclosures}

The authors have no disclosures to make.

\subsection{Acknowledgements}

The authors would like to thank Drs. Andrea Jurisicova and Rudiger von Harsorf for valuable discussions and insight.

\subsection{Funding}

This work was funded by the Ontario Graduate Scholarship (RC), Heart and Stroke Foundation of Ontario (AOG: T-6281), an unrestricted grant from Boehringer Ingelheim Canada, and a Heart and Stroke/Lewar Centre Fellowship (NB). AOG was a New Investigator of HSF Canada and holds a Canada Research Chair.

\subsection{Author contributions}

R.Chis, P.Sharma, N.Bousette, T. Miyake, A.Wilson, and A.O. Gramolini conceived and designed research; R.Chis, P.Sharma, N.Bousette, T. Miyake, and A.Wilson performed experiments; R.Chis, P.Sharma, N.Bousette, T. Miyake, and A.O. Gramolini analyzed data; R.Chis, P.Sharma, N.Bousette, T. Miyake, A.Wilson, P.H.Backx and A.O. Gramolini interpreted results of experiments; R.Chis, P.Sharma, and A.O. Gramolini prepared figures; R.Chis, P.Sharma, and A.O. Gramolini drafted manuscript; R.Chis, P.Sharma, P.H.Backx, and A.O. Gramolini edited and revised the manuscript; R.Chis, P.Sharma, N.Bousette, T. Miyake, A.Wilson, P.H.Backx and A.O. Gramolini approved the final version of manuscript. 


\subsection{References}

1. Aschkenasy G, Bromberg Z, Raj N, Deutschman CS, and Weiss YG. Enhanced Hsp70 Expression Protects against Acute Lung Injury by Modulating Apoptotic Pathways. Public Library of Science One 6: e26956, 2011.

2. Bennardini F, Wrzosek A, and Chiesi M. Alpha B-crystallin in cardiac tissue. Association with actin and desmin filaments. Circulation Research 71: 288-294, 1992.

3. Bousette N, Chugh S, Fong V, Isserlin R, Kim K-H, Volchuk A, Backx PH, Liu P, Kislinger T, MacLennan DH, Emili A, and Gramolini AO. Constitutively active calcineurin induces cardiac endoplasmic reticulum stress and protects against apoptosis that is mediated by a-crystallin-B. Proceedings of the National Academy of Sciences 107: 18481-18486, 2010.

4. Coutinho ARS, Assumpção MEO, and Bordignon V. Presence of cleaved caspase 3 in swine embryos of different developmental capacities produced by parthenogenetic activation. Molecular Reproduction and Development 78: 673-683, 2011.

5. Downs CA, Jones LR, and Heckathorn SA. Evidence for a Novel Set of Small HeatShock Proteins That Associates with the Mitochondria of Murine PC12 Cells and Protects NADH:Ubiquinone Oxidoreductase from Heat and Oxidative Stress. Archives of Biochemistry and Biophysics 365: 344-350, 1999.

6. Gramolini AO, Kislinger T, Alikhani-Koopaei R, Fong V, Thompson NJ, Isserlin R, Sharma P, Oudit GY, Trivieri MG, Fagan A, Kannan A, Higgins DG, Huedig H, Hess G, Arab S, Seidman JG, Seidman CE, Frey B, Perry M, Backx PH, Liu PP, MacLennan DH, and Emili A. Comparative Proteomics Profiling of a Phospholamban Mutant Mouse Model of Dilated Cardiomyopathy Reveals Progressive Intracellular Stress Responses. Molecular \& Cellular Proteomics 7: 519-533, 2008.

7. Gravance CG, Garner DL, Baumber J, and Ball BA. Assessment of equine sperm mitochondrial function using JC-1. Theriogenology 53: 1691-1703, 2000.

8. Green DR, and Reed JC.. Mitochondria and Apoptosis. Science 281: 1309-1312, 1998.

9. Green DR, and Kroemer G. The Pathophysiology of Mitochondrial Cell Death. Science 305: 626-629, 2004.

10. Hajnóczky G, Csordás G, Madesh M, and Pacher P. Control of apoptosis by IP3and ryanodine receptor driven calcium signals. Cell Calcium 28: 349-363, 2000.

11. Halestrap AP, Clarke SJ, and Javadov SA. Mitochondrial permeability transition pore opening during myocardial reperfusion-a target for cardioprotection. Cardiovascular Research 61: 372-385, 2004.

12. He L, and Lemasters JJ. Regulated and unregulated mitochondrial permeability transition pores: a new paradigm of pore structure and function? Federation of European Biochemical Societies Letters 512: 1-7, 2002. 
13. Hess ML, Manson NH, and Okabe E. Involvement of free radicals in the pathophysiology of ischemic heart disease. Canadian Journal of Physiology and Pharmacology 60: 1382-1389, 1982.

14. Hoover HE, Thuerauf DJ, Martindale JJ, and Glembotski CC. $\alpha \mathrm{B}$-crystallin Gene Induction and Phosphorylation by MKK6-activated p38. Journal of Biological Chemistry 275: 23825-23833, 2000.

15. Ito H, Okamoto K, Nakayama H, Isobe T, and Kato K. Phosphorylation of $\alpha B$ Crystallin in Response to Various Types of Stress. Journal of Biological Chemistry 272: 2993429941, 1997.

16. Jin J-K, Whittaker R, Glassy MS, Barlow SB, Gottlieb RA, and Glembotski CC. Localization of phosphorylated $\alpha \mathrm{B}$-crystallin to heart mitochondria during ischemia-reperfusion. American Journal of Physiology - Heart and Circulatory Physiology 294: H337-H344, 2008.

17. Kamradt MC, Chen F, and Cryns VL. The Small Heat Shock Protein $\alpha$ B-Crystallin Negatively Regulates Cytochrome c- and Caspase-8-dependent Activation of Caspase-3 by Inhibiting Its Autoproteolytic Maturation. Journal of Biological Chemistry 276: 16059-16063, 2001.

18. Kamradt MC, Chen F, Sam S, and Cryns VL. The Small Heat Shock Protein $\alpha B-$ crystallin Negatively Regulates Apoptosis during Myogenic Differentiation by Inhibiting Caspase-3 Activation. Journal of Biological Chemistry 277: 38731-38736, 2002.

19. Kraev A, Chumakov I, and Carafoli E. The Organization of the Human Gene NCX1 Encoding the Sodium-Calcium Exchanger. Genomics 37: 105-112, 1996.

20. Longoni SJ, Chiesi M Cardiac alpha-crystallin. I. Isolation and identification. Molecular and Cellular Biochemistry 99: 113-120, 1990.

21. Maloyan A, Sanbe A, Osinska H, Westfall M, Robinson D, Imahashi K-i, Murphy E, and Robbins J. Mitochondrial Dysfunction and Apoptosis Underlie the Pathogenic Process in $\alpha-$ B-Crystallin Desmin-Related Cardiomyopathy. Circulation 112: 3451-3461, 2005.

22. Mao Y-W, Xiang H, Wang J, Korsmeyer S, Reddan J, and Li DW-C. Human bcl-2 Gene Attenuates the Ability of Rabbit Lens Epithelial Cells against H2O2-induced Apoptosis through Down-regulation of the $\alpha \mathrm{B}$-crystallin Gene. Journal of Biological Chemistry 276: 43435-43445, 2001.

23. Mao YW, Liu JP, Xiang H, and Li DWC. Human alphaA- and alphaB-crystallins bind to Bax and Bcl-XS to sequester their translocation during staurosporine-induced apoptosis. Cell Death and Differentiation 11: 512-526, 2004.

24. Martindale JJ, Wall JA, Martinez-Longoria DM, Aryal P, Rockman HA, Guo Y, Bolli R, and Glembotski CC. Overexpression of Mitogen-activated Protein Kinase Kinase 6 in the Heart Improves Functional Recovery from Ischemia in Vitro and Protects against Myocardial Infarction in Vivo. Journal of Biological Chemistry 280: 669-676, 2005. 
25. Morrison LE, Hoover HE, Thuerauf DJ, and Glembotski CC. Mimicking Phosphorylation of $\alpha \mathrm{B}$-Crystallin on Serine-59 Is Necessary and Sufficient to Provide Maximal Protection of Cardiac Myocytes From Apoptosis. Circulation Research 92: 203-211, 2003.

26. Morrison LE, Whittaker RJ, Klepper RE, Wawrousek EF, and Glembotski CC. Roles for $\alpha \mathrm{B}$-crystallin and HSPB2 in protecting the myocardium from ischemia-reperfusioninduced damage in a KO mouse model. American Journal of Physiology - Heart and Circulatory Physiology 286: H847-H855, 2004.

27. Nakagawa T, Zhu H, Morishima N, Li E, Xu J, Yankner BA, and Yuan J. Caspase12 mediates endoplasmic-reticulum-specific apoptosis and cytotoxicity by amyloid-[beta]. Nature 403: 98-103, 2000.

28. Oskarsson HJ, Coppey L, Weiss RM, and Li W-G. Antioxidants attenuate myocyte apoptosis in the remote non-infarcted myocardium following large myocardial infarction. Cardiovascular Research 45: 679-687, 2000.

29. Ray PS, Martin JL, Swanson EA, Otani H, Dillman WH, and Das DK. Transgene overexpression of $\alpha \mathrm{B}$ crystallin confers simultaneous protection against cardiomyocyte apoptosis and necrosis during myocardial ischemia and reperfusion. The Federation of American Societies for Experimental Biology Journal 15: 393-402, 2001.

30. Sharma P, Shathasivam T, Ignatchenko V, Kislinger T, and Gramolini AO. Identification of an FHL1 protein complex containing ACTN1, ACTN4, and PDLIM1 using affinity purifications and MS-based protein-protein interaction analysis. Molecular BioSystems 7: 1185-1196, 2011.

31. Sharma P. IV, Grace K. , Ursprung C. , Kislinger T. , and Gramolini A.O. . Endoplasmic Reticulum Protein Targeting of Phospholamban: A Common Role for an NTerminal Di-Arginine Motif in ER Retention? Public Library of Science One 5: 2010.

32. Shin J-H, Jeong J-Y, Jin Y, Kim I-D, and Lee J-K. p38 $\beta$ MAPK affords cytoprotection against oxidative stress-induced astrocyte apoptosis via induction of $\alpha \mathrm{B}$-crystallin and its anti-apoptotic function. Neuroscience Letters 501: 132-137, 2011.

33. Slezak Jea. Hydrogen Peroxide Changes in Ischemic and Reperfused Heart. American Journal of Pathology 147: 772-781, 1995.

34. Taylor RP, and Benjamin IJ. Small heat shock proteins: a new classification scheme in mammals. Journal of Molecular and Cellular Cardiology 38: 433-444, 2005.

35. Vandeplassche G, Hermans C, Thoné F, and Borgers M. Mitochondrial hydrogen peroxide generation by NADH-oxidase activity following regional myocardial ischemia in the dog. Journal of Molecular and Cellular Cardiology 21: 383-392, 1989.

36. Velotta JB, Kimura N, Chang SH, Chung J, Itoh S, Rothbard J, Yang PC, Steinman L, Robbins RC, and Fischbein MP. $\alpha$ B-Crystallin Improves Murine Cardiac Function and Attenuates Apoptosis in Human Endothelial Cells Exposed to Ischemia-Reperfusion. The Annals of Thoracic Surgery 91: 1907-1913, 2011. 
37. von Harsdorf R, Li P-F, and Dietz R. Signaling Pathways in Reactive Oxygen SpeciesInduced Cardiomyocyte Apoptosis. Circulation 99: 2934-2941, 1999.

38. Wang X, Perez E, Liu R, Yan L-J, Mallet RT, and Yang S-H. Pyruvate protects mitochondria from oxidative stress in human neuroblastoma SK-N-SH cells. Brain Research 1132: 1-9, 2007.

39. Whelan RS, Kaplinskiy V, and Kitsis RN. Cell Death in the Pathogenesis of Heart Disease: Mechanisms and Significance. Annual Review of Physiology 72: 19-44, 2010.

40. Yan L-J, Christians ES, Liu L, Xiao X, Sohal RS, and Benjamin IJ. Mouse heat shock transcription factor 1 deficiency alters cardiac redox homeostasis and increases mitochondrial oxidative damage. European Molecular Biology Organization Journal 21: 51645172, 2002.

41. Zorov DB, Filburn CR, Klotz L-O, Zweier JL, and Sollott SJ. Reactive Oxygen Species (Ros-Induced) Ros Release. The Journal of Experimental Medicine 192: 1001-1014, 2000. 


\subsection{Figures}

A

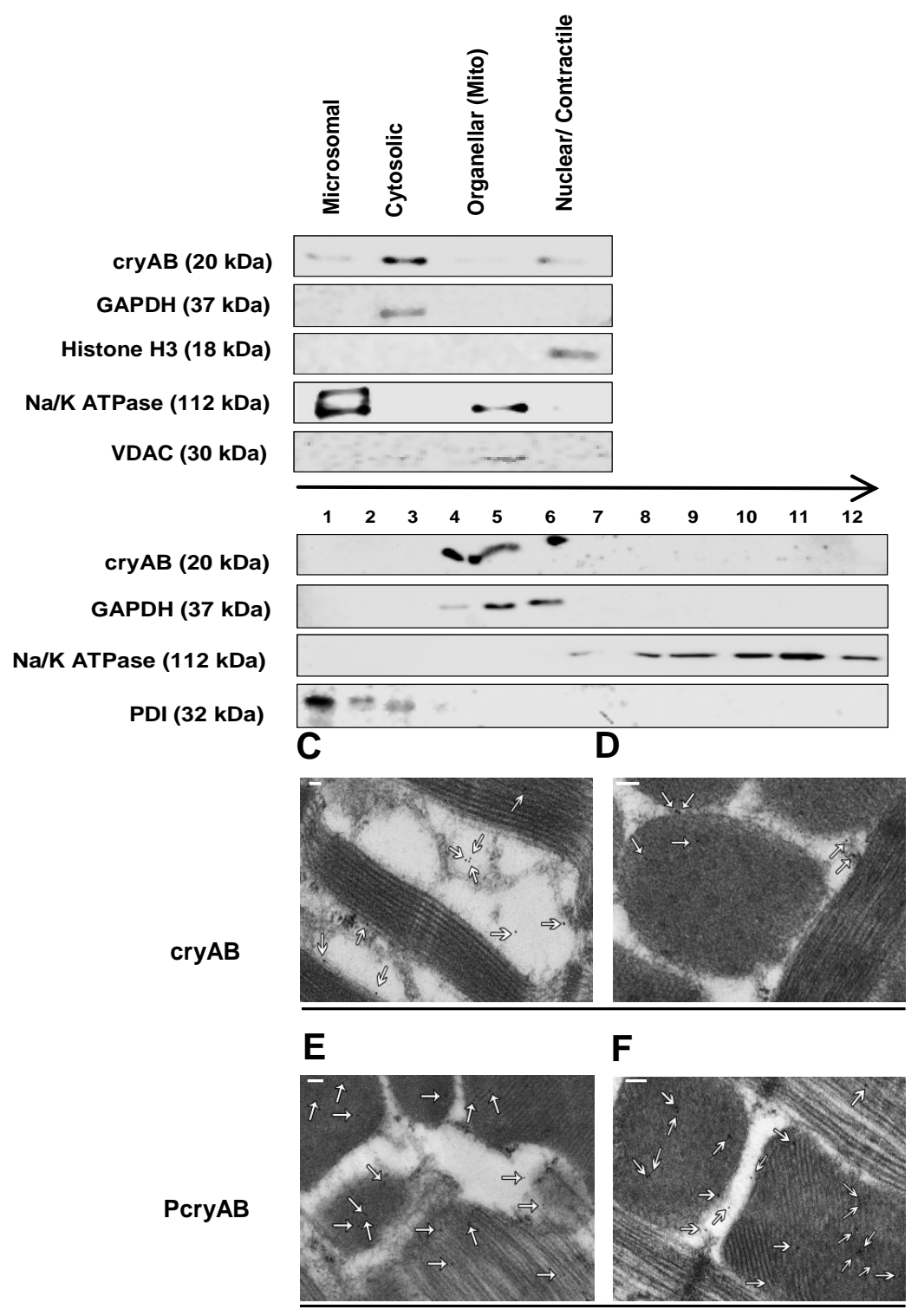

\section{Figure 3. CryAB distribution in cardiac cells.}

(A) Immunoblot of cryAB in different sub-cellular fractions isolated from adult mouse hearts. The distribution of glyceraldehyde 3-phosphate dehydrogenase (GAPDH) as a marker for the cytosolic fraction, Histone $\mathrm{H} 3$ for the nuclear fraction, PDI for the ER, VDAC for the mitochondrial fraction and $\mathrm{Na} / \mathrm{K}$ ATPase for the microsomal fraction was assessed. (B) Sub-cellular fractionation of wild type adult mouse hearts and cell lysates run on a continuous 20-60\% sucrose gradient. Fractions 1-13 contain the highest to lowest sucrose concentrations respectively. The endoplasmic reticulum marker protein disulfide isomerase (PDI), plasma membrane protein marker $\mathrm{Na} / \mathrm{K}$ ATPase and GAPDH as a cytosol protein were detected as markers to monitor the fractionation procedure. A minimum of 3 experiments were performed for each gradient. (C) Electron microscopy images depicting immunogold staining of cryAB, upper panel, and PcryAB, lower panel, under control conditions. Arrows represent gold particles bound to cryAB and PcryAB, respectively. Scale bar is $100 \mathrm{~nm}$. Images are representative of a minimum of 50 separate microscopy fields. 
A

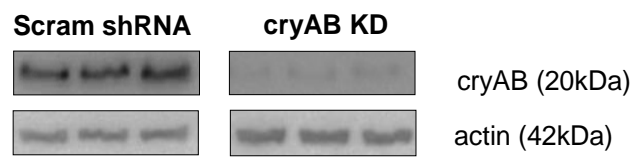

B

C
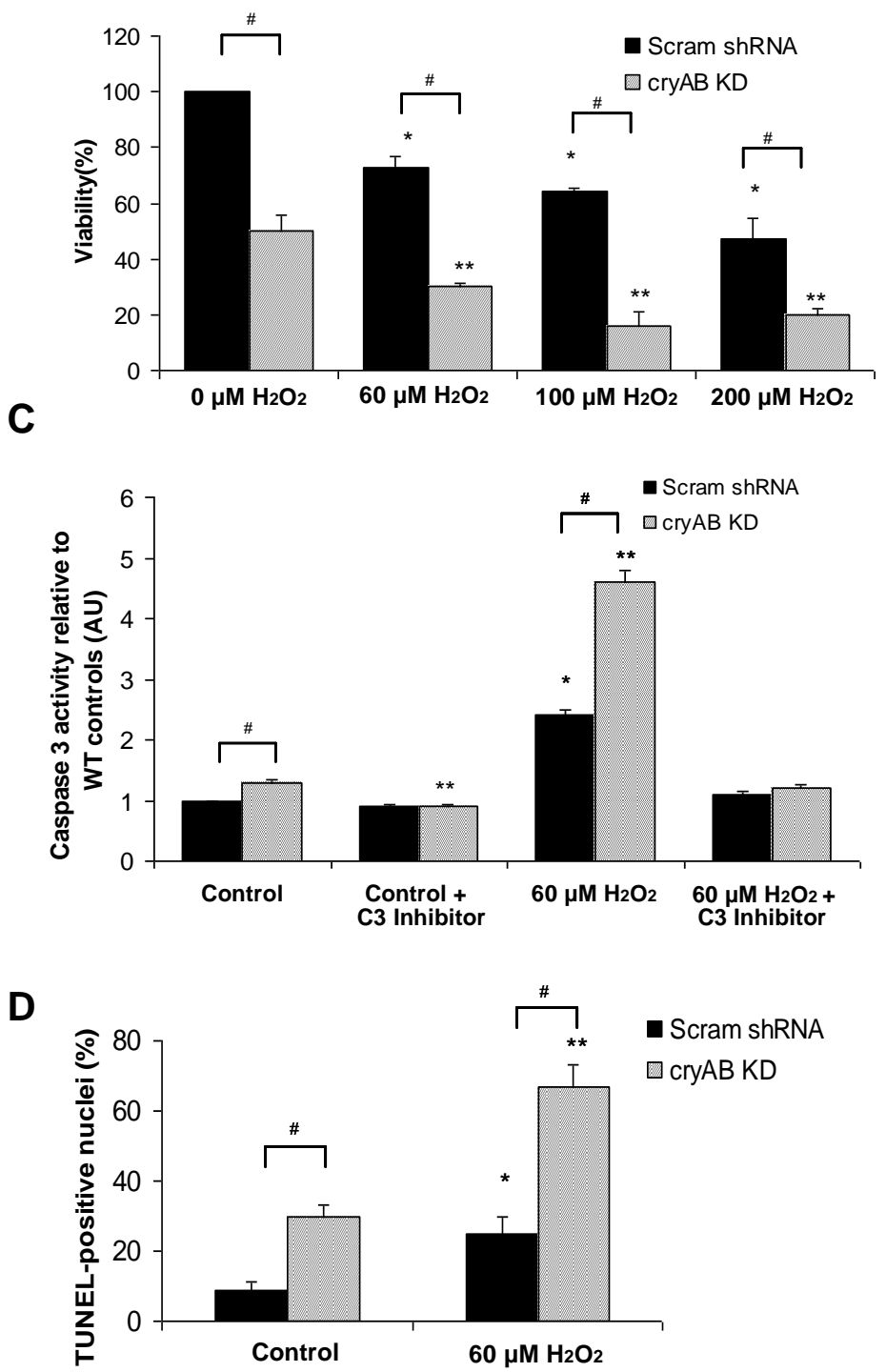

Figure 4. Viability in cryAB-silenced CMs.

(A) Immunoblot for cryAB and actin in CMs either transduced with scrambled shRNAs (Scram shRNA) or cryAB targeting shRNA virus (cryAB KD). In the cryAB KD cells, cryAB levels were $\sim 22 \%$ of the expression levels in CMs transduced with the scrambled control plasmid. (B) Quantification of viability in CMs transduced with cryABtargeting shRNA (cryAB KD) compared to CMs transduced with scrambled shRNA (Scram shRNA) CMs at increasing $\mathrm{H}_{2} \mathrm{O}_{2}$ concentrations (* vs Scram shRNA control at $\mathrm{p}<0.05$ ) and ** vs $\mathrm{KD}$ control at $\mathrm{p}<0.05$ ). Differences between groups (KD vs. Scram) are represented by \# at $\mathrm{p}<0.05$. Values are reported as mean $+\mathrm{SE}$ $(\mathrm{n}=3 /$ treatment). (C) Quantification of caspase 3 activity in Scram (* at $\mathrm{p}<0.05)$ and cryAB-KD CMs (** at $\mathrm{p}$ $<0.05$ ) following exposure to $60 \mu \mathrm{M} \mathrm{H}_{2} \mathrm{O}_{2}$ when compared to control conditions. Differences between groups (Scram vs. KD) are represented by \# at $\mathrm{p}<0.05$. Values reported as mean $+\mathrm{SE}(\mathrm{n}=6 /$ treatment). (D) Quantification of TUNEL-positive nuclei in Scram (* at p < 0.05) and KD CMs (** at p <0.05) following exposure to $60 \mu \mathrm{M} \mathrm{H}_{2} \mathrm{O}_{2}$ compared to control conditions. Differences between groups (KD vs. Scram) are represented by \# at $\mathrm{p}<0.05$. Values reported as mean $+\mathrm{SE}(\mathrm{n}=1000$ cells/treatment $)$. 
A

\begin{tabular}{|c|c|c|}
\hline Control & $100 \mu \mathrm{M} \mathrm{H} \mathrm{O}_{2}$ & \\
\hline--- & & cryAB $(20 \mathrm{kDa})$ \\
\hline$--\cdots$ & $-\infty$ & PcryAB (22kDa) \\
\hline$-\div-$ & $-\div-$ & $\operatorname{actin}(42 \mathrm{kDa})$ \\
\hline
\end{tabular}

B

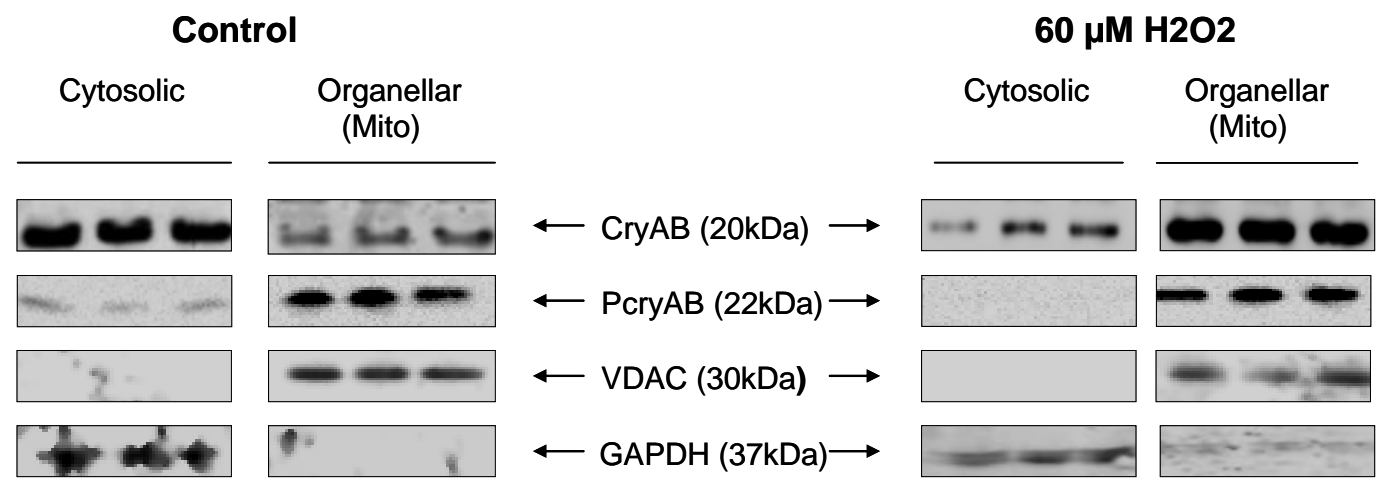

C

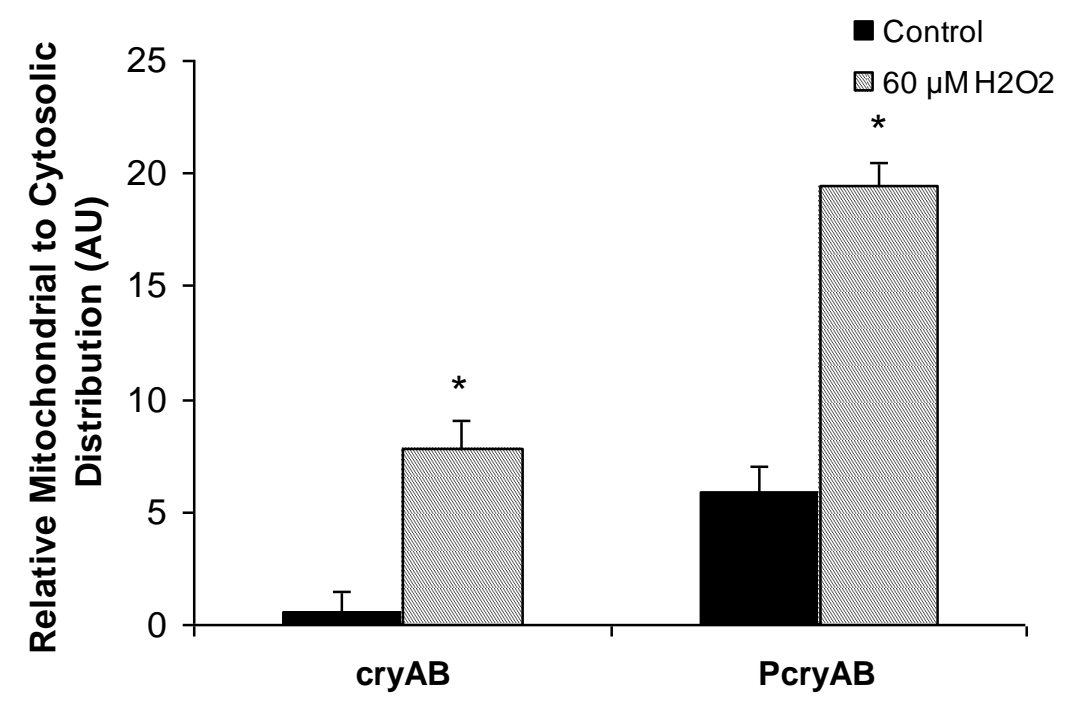

Figure 5. Upregulation and translocation of cryAB and PcryAB to the mitochondria under oxidative stress conditions.

(A) Immunoblots of cry $\mathrm{AB}$ and PcryAB along with actin standards in WT mouse adult cardiac tissue controls or cells exposed to $100 \mu \mathrm{M} \mathrm{H}_{2} \mathrm{O}_{2}$ for 1 hour. (B) Immunoblots of cytosolic and organellar sub-cellular fractions for cryAB and PcryAB in cultured neonatal CMs under control conditions and following exposure to $60 \mu \mathrm{M} \mathrm{H}_{2} \mathrm{O}_{2}$. The distribution of VDAC, a mitochondrial marker and GAPDH, a cytosolic marker, was assessed to monitor the purity of the fractions. (C) Quantification of the mitochondrial to cytosolic distribution of cryAB and PcryAB in cultured neonatal CMs. The band intensities of cryAB organellar levels were normalized to values observed in cytosolic fractions and are expressed in arbitrary units (A.U.). Values are reported as mean $+\mathrm{SE}, \mathrm{n}=3$, * vs. control conditions at $\mathrm{p}<0.03$ ). 
A

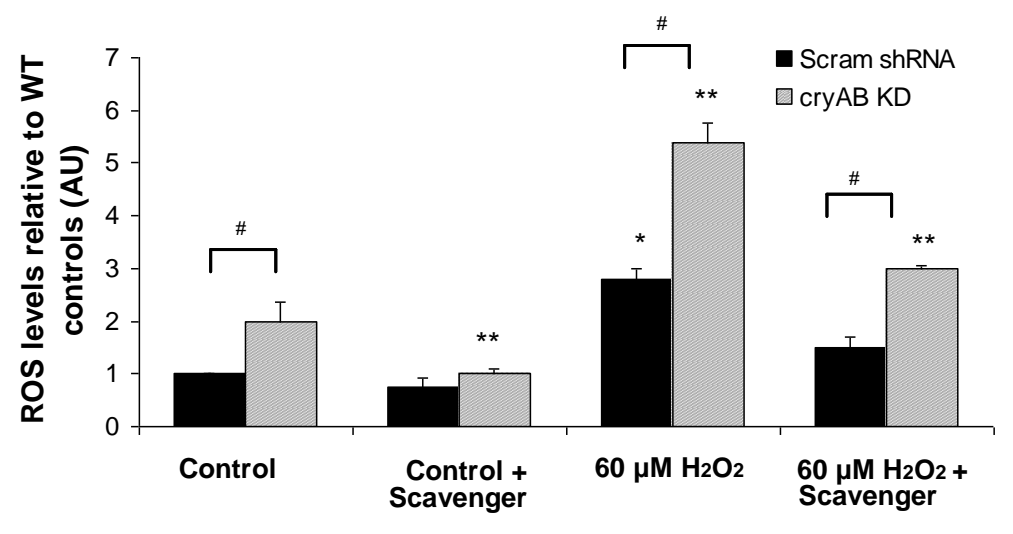

B

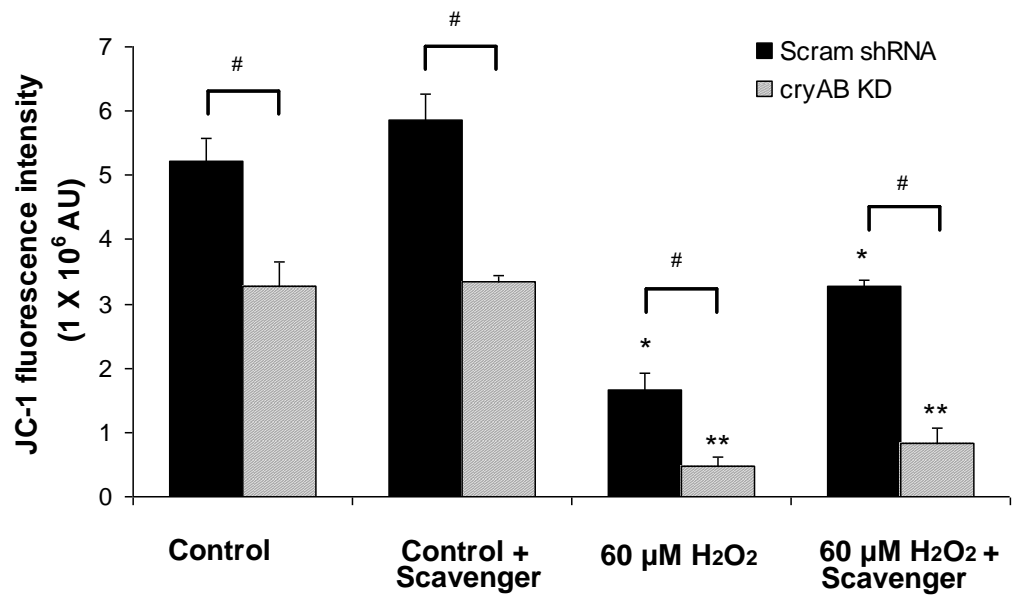

Figure 6. Apoptosis induction in CMs.

(A) Quantification of ROS levels in Scram and cryABKD CMs at baseline and following exposure to $\mathrm{H}_{2} \mathrm{O}_{2}$ (* vs. Scram controls and $* *$ vs. cryAB-KD controls at $\mathrm{p}<0.05$ ). ROS were also assessed following treatment of cells with ROS scavengers, Tiron and sodium pyruvate. Differences between groups (cryAB-KD vs. Scram) are represented by \# at $\mathrm{p}<0.05$. Values reported as mean $+\mathrm{SE}(\mathrm{n}=6 /$ treatment). (B) Quantification of JC-1 red fluorescence using a plate reader. JC-1 fluorescence was assessed in Scram and KD CMs following exposure to 60 $\mu \mathrm{M} \mathrm{H}_{2} \mathrm{O}_{2}$ in Scram CMs (* at $\left.\mathrm{p}<0.05\right)$ and cryAB-KD CMs (** at $\left.\mathrm{p}<0.05\right)$ when compared to control conditions. 
Scram shRNA

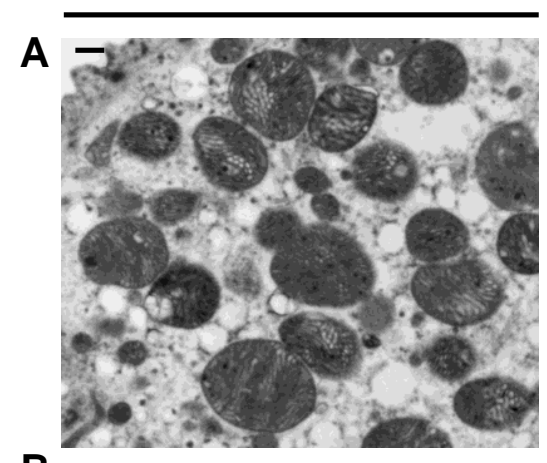

B

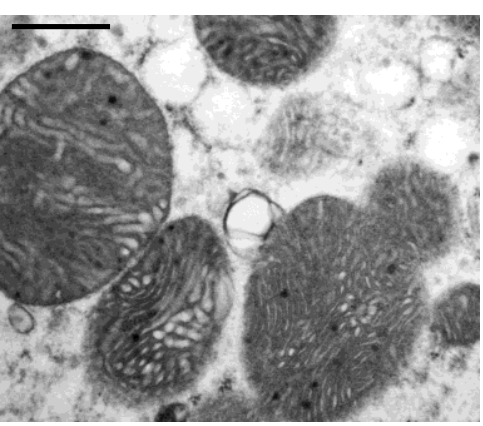

cryAB KD
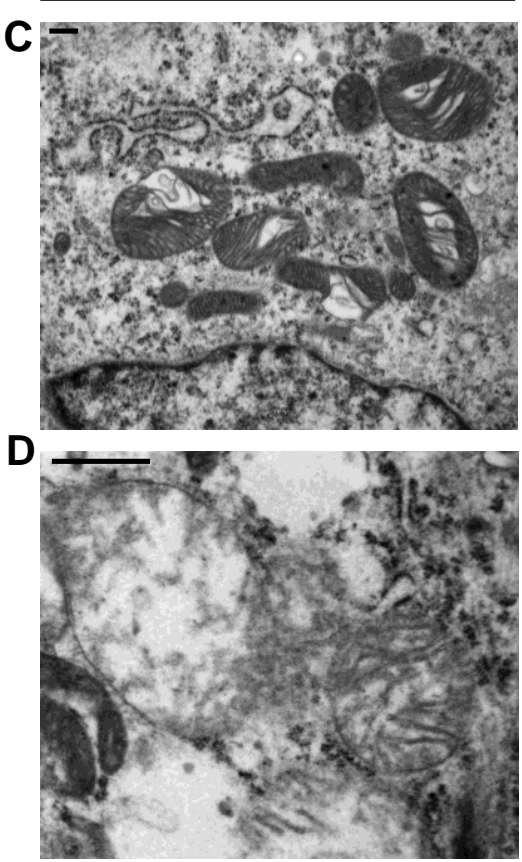

Figure 7. Effect of cryAB silencing on mitochondrial ultrastructure in mouse neonatal CMs.

Transmission electron microscopy (TEM) images of (A) scrambled and (B) cryAB KD CMs examining mitochondria. (C) and (D) are higher magnifications of mitochondria in CMs transduced with scrambled or cryABtargeting shRNAs, respectively. Scale bar indicated corresponds to $500 \mathrm{~nm}$. 


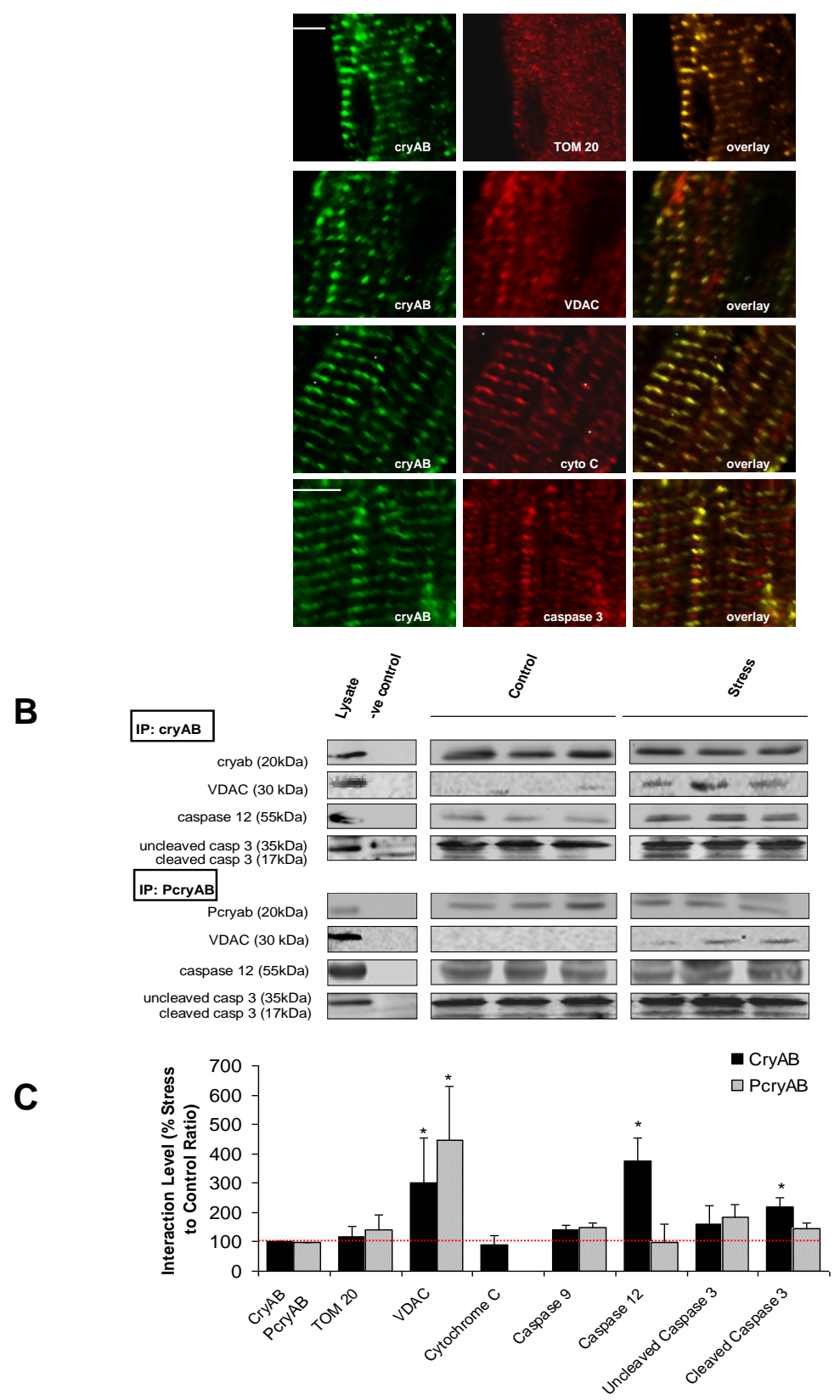

Figure 8. CryAB protein interactions in the apoptotic cascade.

(A) Immunofluorescence of adult mouse CMs using cryAB, TOM 20, VDAC, cytochrome C and caspase 3 antibodies. Far right panel is indicative of co-localization. Scale bars represent $4 \mu \mathrm{m}$. (B) Immunoblots depicting the interactions of cryAB and PcryAB with TOM 20, VDAC, cytochrome C, caspase 9, caspase 12 and caspase 3. CryAB and PcryAB were immunoprecipitated from control and stressed adult cardiac tissue $\left(100 \mu \mathrm{M} \mathrm{H}_{2} \mathrm{O}_{2}\right.$ for 1 hour) using $\mathrm{A} / \mathrm{G}$ agarose beads and the eluate was run on an SDS-page gel, probing for interactors in both control and stressed cardiac tissue. The gels were also probed for cryAB and PcryAB as a control. IgG beads alone were used as negative controls. Equal loading of cardiac lysate is shown. (C) Volumetric quantification of the ratio of stressed to control levels of interaction between cryAB (upper blot) and PcryAB (lower blot) and TOM 20, VDAC, cytochrome $C$, caspase 9, caspase 12 and caspase 3 relative to cryAB and PcryAB input levels $(*$ at $p<0.05)$. Values are reported as mean $+\mathrm{SE}(\mathrm{n}=3 /$ treatment $)$. 


\section{CHAPTER FIVE: DISCUSSION}

In the present study, I demonstrated the following: (1) under control conditions cryAB assumes a cytosolic localization in adult mouse $\mathrm{CMs}$ and that in response to hydrogen peroxide-induced oxidative stress, an in vitro mimic of $\mathrm{I} / \mathrm{R}$, cryB and PcryAB translocate to the mitochondria, (2) following $\mathrm{H}_{2} \mathrm{O}_{2}$ exposure total cryAB levels become upregulated and knock-down of cryAB leads to a significant reduction in viability in mouse neonatal cryAB KD CMs suggesting that cry $A B$ is protective against apoptosis, (3) cryAB silencing is accompanied by significant ROS increases, mitochondrial ultrastructure alterations and dissipation of membrane potential, leading to a significant reduction in viability in mouse neonatal $\mathrm{CMs}$, by increasing the levels of intrinsic apoptosis at baseline conditions, (4) cryAB interacts with a number of apoptotic mediators, TOM 20, VDAC, cytochrome $\mathrm{C}$, and caspases 9,12 and 3 under both control conditions and following exposure to $\mathrm{H}_{2} \mathrm{O}_{2}$ and (5) a significantly higher level of interaction following exposure to $\mathrm{H}_{2} \mathrm{O}_{2}$ when compared to control conditions was observed between both cryAB and PcryAB and VDAC and between cryAB and caspase 12, suggesting these interactions may be part of the cry $\mathrm{AB}$ protective mechanism against apoptosis in CMs.

\subsection{CryAB localization and translocation in cardiomyocytes}

CryAB is a small heat shock protein that has cardio-protective properties ${ }^{87}$. It has been shown that part of the protective mechanism of cryAB involves its translocation to the mitochondria, thus reducing $\mathrm{I} / \mathrm{R}$ damage in mouse hearts during ex vivo $\mathrm{I} / \mathrm{R}^{100}$. Moreover, a molecular mimic of PcryAB enhances the protective ability of cryAB against several different stresses that imitate $I / R^{87}$. Our results extend these earlier findings to an in vitro model of $I / R$ in cultured neonatal mouse CMs.

\subsubsection{Ischemia/Reperfusion injury and hydrogen peroxide in vitro}

Myocardial ischemia is caused by an inadequate blood supply, and thereby an inadequate supply of oxygen and glucose to the heart, leading to $\mathrm{CM}$ death if blood flow is not rapidly restored ${ }^{21}$. Reperfusion of ischemic tissue has its dangers as well, as it is also associated with cardiac tissue injury $^{22}$.Reactive oxygen species are known to play a major role in the pathogenesis of myocardial dysfunction in a variety of conditions including $\mathrm{I} / \mathrm{R}$ injury ${ }^{110}$. Among different 
activated oxygen species, $\mathrm{H}_{2} \mathrm{O}_{2}$, which is not a free radical, has a relatively longer half-life and may play a significant role in oxidative stress injury ${ }^{111}$. Different oxidases of the heart, especially xanthine oxidase, monoamine oxidase, and NADH oxidase produce $\mathrm{H}_{2} \mathrm{O}_{2}$, which directly and/or indirectly through the production of highly reactive hydroxyl radicals can cause myocardial injury $^{111}$. Global ischemia as well as reperfusion have been associated with significant increases in myocardial $\mathrm{H}_{2} \mathrm{O}_{2}$ content ${ }^{112}$. As a result, the addition of $\mathrm{H}_{2} \mathrm{O}_{2}$ to cells in culture provides a model for the study of I/R injury in vitro. Furthermore, I/R has been associated with the induction of apoptosis in the myocardium ${ }^{23,24,25} \cdot \mathrm{H}_{2} \mathrm{O}_{2}$ has also been shown to lead to apoptosis in CMs, by activating the intrinsic pathway of apoptosis ${ }^{51}$. Its abundant presence in I/R injury and its ability to induce apoptosis in CMs led us to use optimally determined levels of $\mathrm{H}_{2} \mathrm{O}_{2}$ in our in vitro model of $\mathrm{I} / \mathrm{R}$ injury to determine the anti-apoptotic mechanism of cryAB in CMs.

\subsubsection{CryAB translocates from the cytosol to the mitochondria following oxaidative stress}

Following sub-cellular fractionation of adult mouse hearts, by differential centrifugation and sucrose gradient separation, generating nuclear, organellar (including the mitochondria), microsomal, and cytosolic fractions, we found that under control conditions, a majority of cryAB (83\%, Fig. 4) was found in the cytosolic fraction, in agreement with the literature ${ }^{113}$. It has been shown previously that under conditions of $\mathrm{I} / \mathrm{R}$, cryAB translocates to the mitochondria and contractile units $^{114}$. Also, following I/R stress, phosphorylation of cryAB on the serine-59 residue enhances protection against apoptosis ${ }^{114}$. Following sub-cellular fractionation and immunofluorescence analysis, cryAB and PcryAB seemed to translocate to the mitochondria. These results suggest that part of the protective mechanism of cryAB in the face of $\mathrm{H}_{2} \mathrm{O}_{2-}$ induced cell death involves its translocation and the translocation of any phosphorylated pools of cryAB to the mitochondria.

With regards to the function of the association of cryAB and PcryAB with the mitochondria, it is possible that, as a molecular chaperone, it exerts its protective effects by binding to other proteins in or on the mitochondria. For instance, PcryAB is associated with the mitochondria even under control conditions (Fig. 3 and 5) and this association increases significantly under conditions of oxidative stress. It is possible that this association is due to PcryAB binding and stabilizing or modulating the activity of MPTP proteins. This speculation is supported by studies 
that have shown that over-expression of PcryAB in rat CMs decreases calcium-induced swelling of the mitochondria, which is used to examine permeability transition pore opening ${ }^{115}$.

A question that remains to be answered involves the nature of the stimulus that draws cryAB and PcryAB to the mitochondria, as cryAB lacks a mitochondrial targeting sequence. Potential stimuli that attract cryAB and PcryAB to the mitochondria could include the unfolding of mitochondrial proteins. For example, TOM 20, a member of the outer mitochondrial membrane protein translocase is prone to unfolding in response to $\mathrm{I} / \mathrm{R}$ in the myocardium ${ }^{47}$. By maintaining TOM 20 structure, other hsps (hsp 70) contribute to protection of the myocardium in ischemic preconditioning $^{47}$. Furthermore, the unfolding of proteins in the MPTP may act as a stimulus to attract cryAB and PcryAB to the mitochondria. Consistent with this possibility are studies showing that the unfolding of these proteins contributes to the activation of $\mathrm{MPT}^{116}$. Our results are in agreement with this possibility, namely the ability of PcryAB to interact with the proteins of the MPTP, as we have shown by EM that PcryAB can also enter the mitochondria, in addition to binding to its surface (Fig. 2C). Other studies have also shown that other cytosolic hsps can localize to the interior of the mitochondria. For instance, hsp25 binds to and protects cytochrome Complex I of the mitochondria from oxidative stress in PC12 cells ${ }^{117}$. Our results extend the findings that hsps can interact with MPTP proteins and stabilize the mitochondria, as they indicate that the interaction of cryAB or PcryAB with VDAC may be important for the protective mechanism, as it is significantly increased under stress conditions (Fig. 7). VDAC is one of the proteins that make up the MPTP, the opening of which induces apoptosis ${ }^{49}$. By interacting with $\mathrm{VDAC}$, cryAB and/or PcryAB may stabilize the mitochondrial membrane, preventing the opening of the pore and thus blocking the first step in the mitochondrial pathway of apoptosis. Therefore, the interaction of cryAB with proteins in the inner mitochondrial membrane may be a central mechanism by cryAB prevents apoptosis. This contention would work in concert with previously established interactions of cryAB with pro-apoptotic proteins Bax and Bcl-Xs, preventing their translocation from cytosol into mitochondria, thus maintaining the mitochondrial integrity and leading to decreased apoptosis ${ }^{97}$. 


\subsection{CryAB silencing contributes to higher apoptosis levels in CMs}

In in vitro models, it has been shown that cryAB prevents apoptosis induced by various factors including hydrogen peroxide ${ }^{103}$. We found that, much like other heat shock proteins, cryAB is upregulated in response to stress (Fig. 5A) following exposure to hydrogen peroxide, suggesting an involvement in protection against cell death. Furthermore, in cryAB KD CMs viability was significantly decreased at all hydrogen peroxide concentrations, suggestive of the fact that cryAB expression is protective against cell death under baseline conditions and following exposure to $\mathrm{H}_{2} \mathrm{O}_{2}$-induced oxidative stress. Increased ROS production at baseline in KD CMs contributed to the higher levels of cell death due to imposed oxidative stress. The results of this project extend previous findings in a cryAB R120G mouse model of desmin cardiomyopathy. This model presents with alterations in mitochondrial organization, architecture and function, decreased complex I activity in the mitochondria by $50 \%$, as well as alterations in the permeability transition pore, and compromised inner membrane potential, leading to the activation of apoptosis $^{118}$. Since in the presence of a mutated from of cryAB, complex I activity is severely compromised, it may be possible that a similar effect would take place in the absence of cryAB. This would explain the increased ROS levels that were observed in the present study.

The mitochondria are a major source of ROS and oxidative stress, but they are also highly susceptible to ROS damage. Proteins, lipids, and nucleic acids can be altered by ROS, resulting in covalent changes that affect mitochondrial structure and function ${ }^{119}$. It has also been shown that mitochondrial complex I activity is decreased by oxidative stress ${ }^{119}$ and reduced complex I activity has been associated with numerous protein misfolding and neurodegenerative diseases $^{120}$. Complex I dysfunction and the subsequent impairment of mitochondrial respiration activate the intrinsic apoptotic pathway by directly triggering the release of cytochrome $\mathrm{C}$ from affected mitochondria ${ }^{121}$. Hence, KD of cryAB could contribute to increased mitochondriainduced apoptosis by ROS-induced ROS production ${ }^{122}$ leading to apoptosis. Electron microscopy images in this study support this speculation, as they show significantly altered mitochondrial ultrastructure in cryAB-silenced cardiomyocytes, suggestive of mitochondrial permeabilization and rupture (Fig. 7), which would lead to apoptosis induction. The toxic effects of ROS can be reduced by scavengers, and there is indeed a reduction of ROS levels and mitochondrial dysfunction with the addition of ROS scavengers (Fig. 6). Given the ability of 
antioxidants to protect against apoptosis in I/R injury by blocking the increased expression of p53, Bax, and caspase- 3 , and inhibiting caspase- 3 activation $^{68}$, it may be that the oxidative stress imposed by cryAB KD functions by the opposite mechanism, increasing the expression of proapoptotic proteins and stimulating apoptosis. And in the permanent absence of cryAB the expectation would be a continuous production of mitochondrial ROS, which would lead to considerable mitochondrial damage. The exogenous addition of hydrogen peroxide caused further reductions in viability that were more pronounced in the KD CMs (Fig.4), suggesting that the KD cells were significantly more prone to undergoing both early apoptosis and mid-stage apoptosis, as depicted by caspase 3 activity under both baseline and stress conditions, indicating that presence of cryAB is protective against apoptosis.

\subsection{Protective mechanism of cryAB in CMs}

The mechanism of protection of cyAB has not been fully elucidated in CMs. CryAB has been shown to directly interact with the precursors of caspase-3 to suppress its activation in an immortalized rabbit lens epithelial cell line ${ }^{103}$ and in breast carcinoma cells ${ }^{104}$. Furthermore, cryAB interacts with pro-caspase 3 to negatively regulate apoptosis during myogenic differentiation $^{98}$. CryAB also interacts with the pro-apoptotic proteins Bax and Bcl-Xs, preventing their translocation from cytosol into mitochondria, thus leading to decreased apoptosis in human lens epithelial cells ${ }^{97}$.

CryAB co-localized with TOM 20, VDAC, cytochrome $\mathrm{C}$ and caspase 3 (Figure 8A), suggesting a potential interaction between cryAB and these proteins following exposure to hydrogen peroxide. Co-immunoprecipitation results revealed that cryAB interacts with numerous proteins, TOM 20, VDAC, cytochrome C, and caspase 9, 12 and 3 under both control conditions and following exposure to hydrogen peroxide (Fig. 7 and 18). As was previously mentioned, cryAB is the most abundantly expressed small heath shock protein in cardiac muscle ${ }^{81}$ and is hence is involved in protein quality control ${ }^{123}$. As a result, its interaction with a high number of proteins is not surprising. What is significant about these results, however, is the apparent higher level of interaction under stress conditions when compared to control conditions of cryAB and PcryAB with VDAC of cryAB with caspase 12 and of cryAB and PcryAB with uncleaved caspase 3, although this did not reach statistical significance (Fig. 7B-C). These results suggest that the 
interaction of cryAB and PcryAB with TOM 20 and caspase 9 may not contribute to the protective mechanism of cryAB, as they are similar under control and stress conditions. The interaction of cry $\mathrm{AB}$ with cytochrome $\mathrm{C}$ is also similar under both control and stress conditions, suggesting that this interaction may also be inconsequential to its protective mechanism. Furthermore, there was no interaction of PcryAB with cytochrome C. This difference could be explained by the different distribution of cryAB and PcryAB. Following exposure to stress, some unphosphorylated cryAB would remain in the cytosol capable of interacting with cytochrome $\mathrm{C}$ to prevent the progression of apoptosis. PcryAB levels, on the other hand, are expected to increase following stress when PcryAB associates mainly with the mitochondria. Following mitochondrial outer membrane rupture due to oxidative stress, cytochrome $\mathrm{C}$ would be found in the cytosol ${ }^{49}$ and PcryAB in the mitochondria, making an interaction between the two less likely than an interaction between unphosphorylated cryAB, some of which is still found in cytosol, as was shown in Figure 5. The interaction between cryAB and PcryAB with uncleaved caspase 3, although it did not reach statistical significance, may be part of the protective mechanism, by preventing the cleavage and activation of caspase 3 thus preventing progression to apoptosis. Furthermore, cryAB has been shown to interact with uncleaved caspase 3 in a breast cancer cell line ${ }^{124}$. The significantly higher interaction of cryAB with cleaved caspase 3 that was observed in the present study, has not been reported previously, to my knowledge. This may be indicative of a potential inhibition of the proteolytic activity of caspase 3 by cryAB even following its cleavage and activation. Therefore, cryAB binding of caspase 3 would prevent its proteolytic cleavage of substrates in the cell and contribute to diminished levels of apoptosis, thus providing an additional point for intervention in preventing apoptosis in CMs.

The interaction of cryAB and PcryAB with VDAC seems to be important to the protective mechanism, as it is significantly increased under stress conditions. VDAC is one of the proteins that make up the mitochondrial permeability transition pore, the opening of which induces apoptosis $^{49}$. By interacting with VDAC, cryAB and PcryAB may help stabilize the mitochondrial membrane, preventing the opening of the pore and thus preventing the early stages of apoptosis. In agreement with this possibility are studies showing that one mechanism by which mitochondrial permeability transition (MPT) is activated is the unfolding of MPT pore proteins upon stress ${ }^{116}$. Furthermore, Maloyan et al. have previously shown that in the desmin cardiomyopathy model, VDAC interacts with the mutated R120G cryAB, whereas there is no 
interaction of VDAC with cryAB in WT hearts ${ }^{118}$. This suggests that cryAB does not interact with VDAC under control conditions, but following the induction of stress (either due to the R120G mutation or exposure to hydrogen peroxide) cryAB can translocate to the mitochondria and interact with VDAC to either stabilize or destabilize the MPT pore.

CryAB, but not PcryAB interaction with caspase 12 is significantly different under stress when compared to control conditions. This suggests that cryAB is also potentially involved in preventing ER stress-induced apoptosis, since ER stress activates ER resident caspase $12{ }^{125}$. This again, is in line with the cytosolic localization of both cryAB and caspase 12 and the mostly mitochondrial localization of PcryAB. Following exposure to hydrogen peroxide, cryAB may interact with caspase 12 in the cytosol, thus preventing the cleavage and activation of caspase 3 by caspase 12 and inhibiting the progression of the apoptosis cascade.

In summary, I found that the protective effects of cryAB in the heart are mediated by its translocation from the cytosol to the mitochondria and its phosphorylation under conditions of stress. Furthermore, we showed that the interaction of cryAB interactions with VDAC, caspase 12 and caspase 3 may be part of the cryAB protective mechanism against apoptosis following exposure to $\mathrm{H}_{2} \mathrm{O}_{2}$ in the heart, thus showing a protective role of cryAB against intrinsic and ERmediated apoptosis. Further studies are needed to delineate the involvement of cryAB in the extrinsic pathway of apoptosis and alternate death pathways, like autophagy and necrosis.

Regulating the levels of apoptosis is an attractive target for ameliorating any ischemic injury in the heart. Our results contribute to delineating the involvement of cryAB in the intrinsic and ER stress pathway of apoptosis activated by ROS-induced oxidative damage. Given the involvement of ROS and $\mathrm{H}_{2} \mathrm{O}_{2}$ in myocardial dysfunction ${ }^{110}$ determining where in the apoptotic cascade cry $\mathrm{AB}$ intervenes to prevent $\mathrm{H}_{2} \mathrm{O}_{2}$ injury can have therapeutic implications for cardiac disease. Further studies focusing on the extrinsic pathway of apoptosis and alternate death pathways, like autophagy and necrosis, would therefore prove a worthwhile pursuit. 


\section{Death Receptor Pathway}

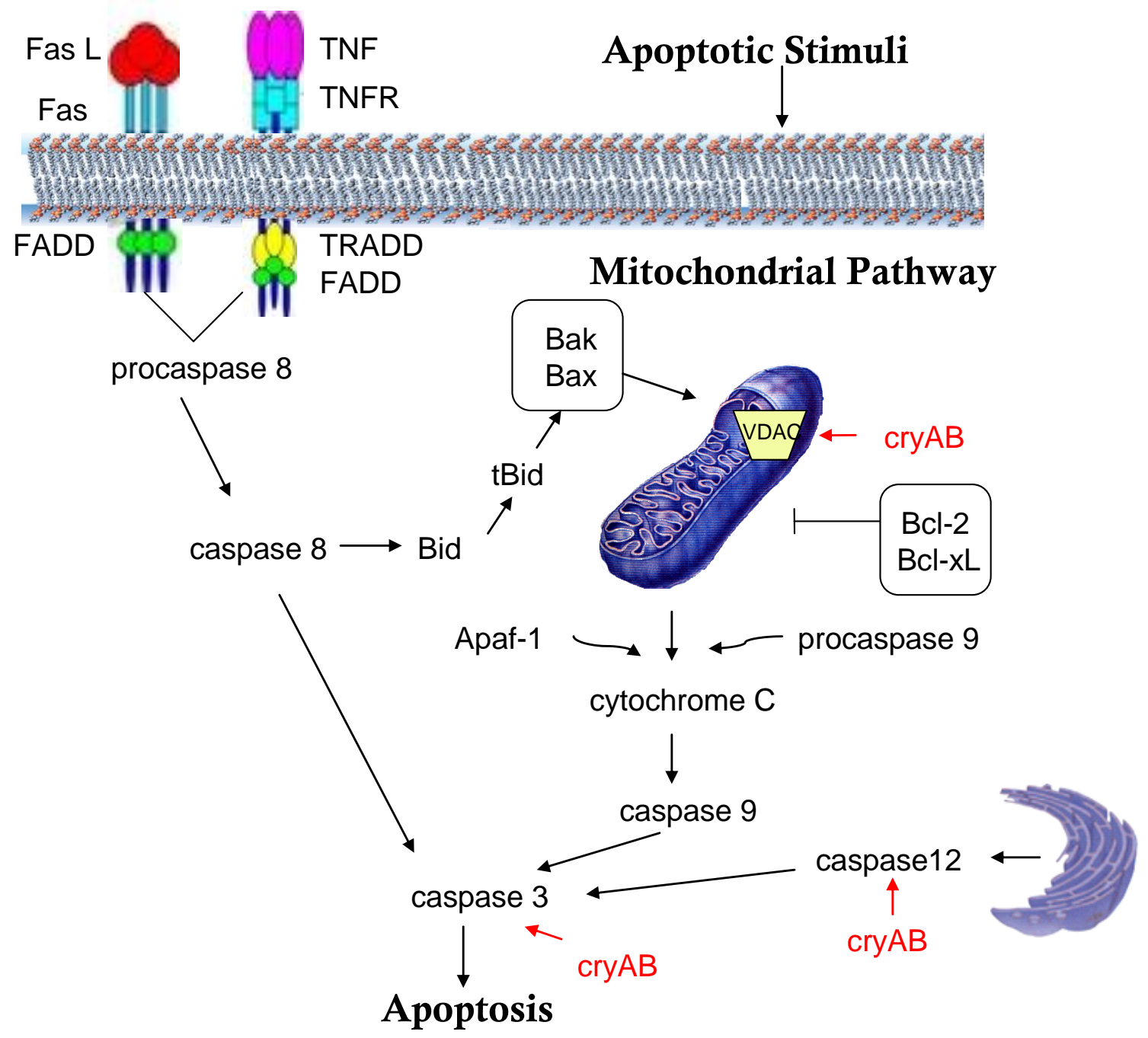

Figure 9. Schematic of cryAB protective mechanism.

The interaction of cryAB and PcryAB with TOM 20 and caspase 9 may not contribute to the protective mechanism of cry $\mathrm{AB}$, as they are similar under control and stress conditions. The interaction of cryAB with cytochrome $\mathrm{C}$ is also similar under both control and stress conditions, suggesting that this interaction may also be inconsequential to its protective mechanism. The interaction between cryAB and PcryAB with uncleaved caspase 3 , is higher under stress conditions and although it did not reach statistical significance, may be part of the protective mechanism, by preventing the cleavage and activation of caspase 3 . The interaction of cryAB with cleaved caspase 3 suggests that cryAB may intervene even following cleavage and activation of caspase 3 and prevent its proteolytic cleavage of substrates. The interaction of cryAB and PcryAB with VDAC may be part of the protective mechanism, as it is significantly increased under stress conditions. By interacting with VDAC, cryAB and PcryAB may help stabilize the mitochondrial membrane, preventing the opening of thepore and thus preventing the early stages of apoptosis. CryAB, but not PcryAB interaction with caspase 12 is significantly higher under stress when compared to control conditions, suggesting a protective effect against ER stress-induced apoptosis. 


\section{CHAPTER SIX: LIMITATIONS}

The current study made use of lentivector mediated knock-down of cryAB in neonatal CMs to determine the protective ability and mechanism of protection of cryAB. Initially, I was also planning on validating the enhanced protective ability of cryAB following its over-expression. However, following transduction with lentivectors containing the cryAB over-expression construct, I was unable to achieve up-regulation of cryAB and therefore unable to test the hypothesis that higher, exogenous cryAB levels would attenuate apoptosis by interacting more readily with proteins in the apoptotic cascade.

And while I began using cultured neonatal CMs from calcineurin transgenic mice (which present with an upregulation of cryAB in cardiac tissue) as an alternative to the over-expression model, it was impossible to attribute any protective effects noticed in the calcineurin $\mathrm{CMs}$ to cryAB alone, due to confounding effects and numerous other changes induced by calcineurin overexpression.

Another limitation relates to the use of an in vitro model. Cells in culture are not the best representation of in vivo cells because cells in culture are situated in a different microenvironment. This became evident throughout our experiments, as we noticed that the cell-culture environment alone exposed the cells to increased oxidative stress at baseline conditions. Furthermore, in in vivo models compensatory effects could be noticed due to redundant genes and proteins. Therefore, the increased cell death observed in our in vitro model could be an over-estimate. For instance, in vivo knock out of cryAB was not associated with increased mortality at baseline conditions. We, however, noticed significantly higher cell death of the KD CMs in the absence of a stressor. Extrapolating from these results, with increased CMs death, we would expect increased likelihood of progression to heart failure in vivo even at baseline. This suggests that in in vivo models our results would be attenuated due to compensatory effects of other genes and proteins. To make up for this limitation, we were initially planning on testing all of our hypotheses on an in vivo mouse model with cardiac knockdown or over-expression of cryAB, achieved through the direct injection of adeno-associated virus serotype 9 (AAV9) containing either the knockdown or over-expression cryAB construct into the hearts of adult mice. AAV9 have a high propensity to the heart (90\%) and their expression is indefinite. Preliminary results of over-expression of GFP-tagged constructs were very promising (data not shown) but due to time constraints, this objective was not pursued further, although it remains very worthwhile for future studies. 
A major drawback for a large portion of this study was the limited CM yield, due to inefficient harvesting techniques. Neonatal CMs isolation was very challenging throughout this study. Due to the fact that we were working with primary cells with no replicative capability, large numbers of pups were euthanized every week to isolate CMs for this study. And despite numerous optimizations to the protocol, the CMs yield was low with every harvest, making protein isolation difficult. The initial goal of the study was to perform all experiments in cultured neonatal CMs. However, due to this limitation, I was unable to do so. The knock-downs and viability assays were performed in neonatal CMs, since the lentivector transductions were optimized and efficient in neonatal CMs. However, I was unable to isolate enough protein from cultured CMs to perform immunoprecipitation. Therefore, I used adult CMs to address the low protein yield. Due to this limitation I was also unable to perform immunoprecipitation out of $\mathrm{KD} \mathrm{CMs}$ as a control and all immunoprecipitaitons were performed in WT CMs.

Lastly, a significant portion of this study relied on the measurement of cell viability, not cell death (with the exception of TUNEL labeling). Cell viability, caspase 3 and mitochondrial assay measurements are dependent on the number of live cells. A limitation of this study was the inability to maintain constant numbers of live cells under control and stress conditions. Due to an inability to maintain constant cell numbers following transduction and stress (since the KD CMs were already more sensitive at baseline and potentially more prone to demise), an underestimate of caspase 3, ROS and JC-1 levels would be expected. This fact could potentially explain why the effects of cryAB KD and exposure to extrinsic oxidative stress were additive on cell viability. This limitation could be overcome by either measuring cell death as an alternative (or addition) to cell viability measurements or by labeling the number of cells present in an assay (for instance, DAPI labeling) and finding the proportion of live cells in different conditions. 


\section{CHAPTER SEVEN: ONGOING WORK}

Since there was a significant decrease in viability in the KD CMs at baseline due to oxidative stress I am also trying to over-express cry $\mathrm{AB}$ in neonatal cardiomyocytes (an initial objective of this project) using Lipofectamine transfection to determine whether over-expression of cryAB would rescue cell viability following treatment with extrinsic hydrogen peroxide, which would mimic the oxidative stress observed at baseline in the absence of cryAB.

I am also using caspase 9 inihibitors and measuring caspase 3 activity to determine whether mitochondrial ROS in the cryAB KD CMs are activating the intrinsic pathway of apoptosis. This would further elucidate the mechanism by which cry $\mathrm{AB}$ silencing induces $\mathrm{CM}$ death. 


\section{CHAPTER EIGHT: FUTURE DIRECTIONS}

In the present study, I characterized the steps at which cryAB intervenes in the apoptotic cascade to attenuate apoptosis in the face of hydrogen peroxide-induced oxidative stress in CMs. For a more complete picture, future directions in which the project could be taken involve the determination of cry $\mathrm{AB}$ involvement in extrinsic apoptosis pathway, as well as other types of cell death, like autophagy in the $\mathrm{H}_{2} \mathrm{O}_{2}$ oxidative stress model. Possible avenues include determining whether autophagosomes can be detected in cryAB WT and KD CMs and whether there is an association of cryAB with autophagosomes.

Furthermore, this study identifies a higher level of interaction of cryAB with caspase 12 under stress conditions when compared to control conditions, suggesting that this interaction may be part of the protective mechanism of cryAB. Caspase 12 becomes active in response to ER stress. Therefore, examining ER stress markers in cryAB KD CMs would be of value. Also, determining the contribution of ER stress to the levels of apoptosis detected by this study, by specifically inhibiting caspase 12 , would be of interest.

ER stress leads to the release of $\mathrm{Ca}^{2+}$ from the ER causing depletion of ER calcium and activating $\mathrm{Ca}^{2+}$-dependent endonucleases involved in DNA fragmentation. Furthermore, $\mathrm{Ca}^{2+}$ is also believed to modulate cytochrome $\mathrm{C}$ release directly by regulating the MPTP, a proposed mechanism for cytochrome $\mathrm{C}$ release during apoptosis. Hence, measuring $\mathrm{Ca}^{2+}$ transients in cryAB KD CMs would confirm the role of $\mathrm{Ca}^{2+}$ and ER stress in triggering apoptosis in the absence of cryAB.

Lastly, testing all of the hypotheses on an in vivo mouse model with cardiac knockdown or overexpression of cry $\mathrm{AB}$, achieved through the direct injection of adeno-associated virus serotype 9 (AAV9) containing either the knockdown or over-expression cryAB construct into the hearts of adult mice remains a worthwhile pursuit. It may be that due to compensatory effects, the consequences of cry $\mathrm{AB} \mathrm{KD}$ in vivo would not be as dire as in this study. As I mentioned previously, in vivo knock out of cryAB was not associated with increased mortality at baseline conditions. Extrapolating from the significantly higher cell death of the KD CMs at baseline observed in this study, one would expect 
increased likelihood of progression to heart failure in vivo even at baseline. This suggests that in in vivo models the results in this study would be attenuated due to compensatory effects of other genes and proteins, so an in vivo model would be helpful in determining the impact of the findings at the in vitro level. Furthermore, to date, the only knock out mouse model of cryAB is in fact a double knock out, of cryAB and hspB2 genes, which are in very close proximity to each other and driven by the same promoter. This fact makes it impossible to distinguish the contribution of the protein encoded by cryAB from the protein encoded by the hspB2 gene to cyto-protection. Targeted disruption of cry $A B$ in the heart through the use of AAV9 would allow for the separation of the roles of cryAB and hspB2 and it provides an alternative the creation of transgenic mouse lines. 


\section{CHAPTER NINE: REFERENCES}

1. Lloyd-Jones D, Adams RJ, Brown TM, et al. Heart Disease and Stroke Statistics-2010 Update. Circulation. 2010;121(7):e46-e215.

2. Nadal-Ginard B, Kajstura J, Leri A, Anversa P. Myocyte Death, Growth, and Regeneration in Cardiac Hypertrophy and Failure. Circulation Research. 2003;92(2):139150.

3. von Harsdorf R, Poole-Wilson PA, Dietz R. Regenerative capacity of the myocardium: implications for treatment of heart failure. The Lancet. 2004;363(9417):1306-1313.

4. Shen S, Kepp O, Michaud M, et al. Association and dissociation of autophagy, apoptosis and necrosis by systematic chemical study. Oncogene. 2011;30(45):4544-4556.

5. Edinger AL, Thompson CB. Death by design: apoptosis, necrosis and autophagy. Current Opinion in Cell Biology. 2004;16(6):663-669.

6. Gill C, Mestril R, Samali A. Losing heart: the role of apoptosis in heart disease-a novel therapeutic target? The FASEB Journal. 2002;16(2):135-146.

7. Kerr JF, Wyllie AH, Currie AR. Apoptosis: A Basic Biological Phenomenon with Wideranging Implications in Tissue Kinetics. British Journal of Cancer. 1972;26(4):239257.

8. Thornberry NA, Lazebnik Y. Caspases: Enemies Within. Science. 1998;281(5381):13121316.

9. Kroemer G, Galluzzi L, Vandenabeele P, et al. Classification of cell death: recommendations of the Nomenclature Committee on Cell Death 2009. Cell Death and Differentiation. 2008;16(1):3-11.

10. Golstein P, Kroemer G. Cell death by necrosis: towards a molecular definition. Trends in Biochemical Sciences. 2007;32(1):37-43.

11. Festjens N, Vanden Berghe $\mathrm{T}$, Cornelis $\mathrm{S}$, Vandenabeele P. RIP1, a kinase on the crossroads of a cell's decision to live or die. Cell Death and Differentiation. 2007;14(3):400-410.

12. Baehrecke EH. Autophagy: dual roles in life and death? Nature Reviews Molecular Cell Biology. 2005;6(6):505-510.

13. Ziegler U, Groscurth P. Morphological Features of Cell Death. Physiology. 2004;19(3):124-128. 
14. Shintani T, Klionsky DJ. Autophagy in Health and Disease: A Double-Edged Sword. Science. 2004;306(5698):990-995.

15. Mizushima N, Yamamoto A, Matsui M, Yoshimori T, Ohsumi Y. In Vivo Analysis of Autophagy in Response to Nutrient Starvation Using Transgenic Mice Expressing a Fluorescent Autophagosome Marker. Molecular Biology of the Cell. 2004;15(3):11011111.

16. Berry DL, Baehrecke EH. Growth Arrest and Autophagy Are Required for Salivary Gland Cell Degradation in Drosophila. Cell. 2007;131(6):1137-1148.

17. Samara C, Syntichaki P, Tavernarakis N. Autophagy is required for necrotic cell death in Caenorhabditis elegans. Cell Death Differ. 2007;15(1):105-112.

18. Narula J, Haider N, Virmani R, et al. Apoptosis in Myocytes in End-Stage Heart Failure. New England Journal of Medicine. 1996;335(16):1182-1189.

19. Guerra S, Leri A, Wang X, et al. Myocyte Death in the Failing Human Heart Is Gender Dependent. Circulation Research. 1999;85(9):856-866.

20. González A, Fortuño MaA, Querejeta R, et al. Cardiomyocyte apoptosis in hypertensive cardiomyopathy. Cardiovascular Research. 2003;59(3):549-562.

21. Nian M, Lee P, Khaper N, Liu P. Inflammatory Cytokines and Postmyocardial Infarction Remodeling. Circulation Research. 2004;94(12):1543-1553.

22. Dong JW, Zhu HF, Zhu WZ, Ding HL, Ma TM, Zhou ZN. Intermittent hypoxia attenuates ischemia/reperfusion induced apoptosis in cardiac myocytes via regulating Bcl-2/Bax expression. Cell Research. 2003;13(5):385-391.

23. Gottlieb RA, Burleson KO, Kloner RA, Babior BM, Engler RL. Reperfusion injury induces apoptosis in rabbit cardiomyocytes. The Journal of Clinical Investigation. 1994;94(4):1621-1628.

24. Fliss H, Gattinger D. Apoptosis in Ischemic and Reperfused Rat Myocardium. Circulation Research. 1996;79(5):949-956.

25. Zhao Z-Q, Nakamura M, Wang N-P, et al. Reperfusion induces myocardial apoptotic cell death. Cardiovascular Research. 2000;45(3):651-660.

26. Saraste A, Pulkki K, Kallajoki M, Henriksen K, Parvinen M, Voipio-Pulkki L-M. Apoptosis in Human Acute Myocardial Infarction. Circulation. 1997;95(2):320-323.

27. Freude B, Masters TN, Robicsek F, et al. Apoptosis is Initiated by Myocardial Ischemia and Executed During Reperfusion. Journal of Molecular and Cellular Cardiology. 2000;32(2):197-208.

28. Piot C, Croisille $\mathrm{P}$, Staat $\mathrm{P}$, et al. Effect of Cyclosporine on Reperfusion Injury in Acute Myocardial Infarction. New England Journal of Medicine. 2008;359(5):473-481. 
29. Pfeffer M, Braunwald E. Ventricular remodeling after myocardial infarction. Experimental observations and clinical implications. Circulation. 1990;81(4):1161-1172.

30. Hilfiker-Kleiner D, Landmesser U, Drexler H. Molecular Mechanisms in Heart Failure: Focus on Cardiac Hypertrophy, Inflammation, Angiogenesis, and Apoptosis. Journal of the American College of Cardiology. 2006:j.jacc.2006.2007.2007.

31. Akyürek Ö, Akyürek N, Sayin T, et al. Association between the severity of heart failure and the susceptibility of myocytes to apoptosis in patients with idiopathic dilated cardiomyopathy. International Journal of Cardiology. 2001;80(1):29-36.

32. Bishopric NH, Andreka P, Slepak T, Webster KA. Molecular mechanisms of apoptosis in the cardiac myocyte. Current Opinion in Pharmacology. 2001;1(2):141-150.

33. Ashkenazi A, Dixit VM. Death Receptors: Signaling and Modulation. Science. 1998;281(5381):1305-1308.

34. Hengartner MO. The biochemistry of apoptosis. Nature. 2000;407(6805):770-776.

35. Ferri KF, Kroemer G. Organelle-specific initiation of cell death pathways. Nature Cell Biology. 2001;3(11):E255-E263.

36. Kroemer G, Reed JC. Mitochondrial control of cell death. Nature Medicine. 2000;6(5):513-519.

37. Lakshmi G. Cell Death Inhibition: Keeping Caspases in Check. Cell. 2001;104(6):805808.

38. Turk B, Turk D, Turk V. Lysosomal cysteine proteases: more than scavengers. Biochimica et Biophysica Acta (BBA) - Protein Structure and Molecular Enzymology. 2000;1477(1-2):98-111.

39. Huang Y, Wang KKW. The calpain family and human disease. Trends in Molecular Medicine. 2001;7(8):355-362.

40. Yamaguchi S, Yamaoka M, Okuyama M, et al. Elevated circulating levels and cardiac secretion of soluble fas ligand in patients with congestive heart failure. The American Journal of Cardiology. 1999;83(10):1500-1503.

41. Kajstura Jea. Apoptotic and necrotic myocyte cell deaths are independent contributing variables of infarct size in rats. Laboratory Investigations. 1996;74(1):86-107.

42. Tanaka M, Ito H, Adachi S, et al. Hypoxia induces apoptosis with enhanced expression of Fas antigen messenger RNA in cultured neonatal rat cardiomyocytes. Circulation Research. 1994;75(3):426-433.

43. Cheng $\mathrm{W}$, Li B, Kajstura J, et al. Stretch-induced programmed myocyte cell death. The Journal of Clinical Investigation. 1995;96(5):2247-2259. 
44. Torre-Amione G, Kapadia S, Lee J, et al. Tumor Necrosis Factor- $\alpha$ and Tumor Necrosis Factor Receptors in the Failing Human Heart. Circulation. 1996;93(4):704-711.

45. Sliwa K, Skudicky D, Candy G, Wisenbaugh T, Sareli P. Randomised investigation of effects of pentoxifylline on left-ventricular performance in idiopathic dilated cardiomyopathy. The Lancet. 1998;351(9109):1091-1093.

46. Krown KA, Page MT, Nguyen C, et al. Tumor necrosis factor alpha-induced apoptosis in cardiac myocytes. Involvement of the sphingolipid signaling cascade in cardiac cell death. The Journal of Clinical Investigation. 1996;98(12):2854-2865.

47. Bowers M, Ardehali H. TOM20 and the Heartbreakers: Evidence for the role of mitochondrial transport proteins in cardioprotection. Journal of Molecular and Cellular Cardiology. 2006;41(3):406-409.

48. Wang X. The expanding role of mitochondria in apoptosis. Genes and Development. 2001;15:2922-2933.

49. Green, John C. Reed DR. Mitochondria and Apoptosis. Science. 1998;281(5381):13091312.

50. Bialik S, Cryns VL, Drincic A, et al. The Mitochondrial Apoptotic Pathway Is Activated by Serum and Glucose Deprivation in Cardiac Myocytes. Circulation Research. 1999;85(5):403-414.

51. von Harsdorf R, Li P-F, Dietz R. Signaling Pathways in Reactive Oxygen SpeciesInduced Cardiomyocyte Apoptosis. Circulation. 1999;99(22):2934-2941.

52. Khosravi-Far R. Death receptor signals to the mitochondria. Cancer Biology \& Therapy. 2004;3(11):1051-1057.

53. Li H, Zhu H, Xu C-j, Yuan J. Cleavage of BID by Caspase 8 Mediates the Mitochondrial Damage in the Fas Pathway of Apoptosis. Cell. 1998;94(4):491-501.

54. Matsuzawa A, Ichijo H. Molecular Mechanisms of the Decision between Life and Death: Regulation of Apoptosis by Apoptosis Signal-Regulating Kinase 1. Journal of Biochemistry. 2001;130(1):1-8.

55. Adams JM, Cory S. Life-or-death decisions by the Bcl-2 protein family. Trends in Biochemical Sciences. 2001;26(1):61-66.

56. Condorelli G, Morisco C, Stassi G, et al. Increased Cardiomyocyte Apoptosis and Changes in Proapoptotic and Antiapoptotic Genes bax and bcl-2 During Left Ventricular Adaptations to Chronic Pressure Overload in the Rat. Circulation. 1999;99(23):30713078.

57. Latif N, Khan MA, Birks E, et al. Upregulation of the Bcl-2 family of proteins in end stage heart failure. Journal of the American College of Cardiology. 2000;35(7):17691777. 
58. Maulik N, Engelman RM, Rousou JA, Flack JE, Deaton D, Das DK. Ischemic Preconditioning Reduces Apoptosis By Upregulating Anti-Death Gene Bcl-2. Circulation. 1999;100(suppl 2):II-369-II-375.

59. Kirshenbaum LA, de Moissac D. The bcl-2 Gene Product Prevents Programmed Cell Death of Ventricular Myocytes. Circulation. 1997;96(5):1580-1585.

60. Maloyan A, Sayegh J, Osinska H, Chua BHL, Robbins J. Manipulation of Death Pathways in Desmin-Related Cardiomyopathy. Circulation Research. 2010;106(9):15241532.

61. Koseki T, Inohara N, Chen S, Núñez G. ARC, an inhibitor of apoptosis expressed in skeletal muscle and heart that interacts selectively with caspases. Proceedings of the National Academy of Sciences. 1998;95(9):5156-5160.

62. Yaoita H, Ogawa K, Maehara K, Maruyama Y. Attenuation of Ischemia/Reperfusion Injury in Rats by a Caspase Inhibitor. Circulation. 1998;97(3):276-281.

63. Gottlieb RA, Gruol DL, Zhu JY, Engler RL. Preconditioning rabbit cardiomyocytes: role of $\mathrm{pH}$, vacuolar proton ATPase, and apoptosis. The Journal of Clinical Investigation. 1996;97(10):2391-2398.

64. Yaoita H, Ogawa K, Maehara K, Maruyama Y. Apoptosis in relevant clinical situations. Cardiovascular Research. 2000;45(3):630-641.

65. Okamura T, Miura T, Takemura G, et al. Effect of caspase inhibitors on myocardial infarct size and myocyte DNA fragmentation in the ischemia-reperfused rat heart. Cardiovascular Research. 2000;45(3):642-650.

66. Mocanu MM, Baxter GF, Yellon DM. Caspase inhibition and limitation of myocardial infarct size: protection against lethal reperfusion injury. British Journal of Pharmacology. 2000;130(2):197-200.

67. Chandra J, Samali A, Orrenius S. Triggering and modulation of apoptosis by oxidative stress. Free Radical Biology and Medicine. 2000;29(3-4):323-333.

68. Oskarsson HJ, Coppey L, Weiss RM, Li W-G. Antioxidants attenuate myocyte apoptosis in the remote non-infarcted myocardium following large myocardial infarction. Cardiovascular Research. 2000;45(3):679-687.

69. Dhalla NS, Elmoselhi AB, Hata T, Makino N. Status of myocardial antioxidants in ischemia-reperfusion injury. Cardiovascular Research. 2000;47(3):446-456.

70. Maulik N, Yoshida T, Das DK. Regulation of cardiomyocyte apoptosis in ischemic reperfused mouse heart by glutathione peroxidase. Molecular and Cellular Biochemistry. 1999;196(1):13-21. 
71. Chen Z, Siu B, Ho Y-S, et al. Overexpression of MnSOD Protects Against Myocardial Ischemia/reperfusion Injury in Transgenic Mice. Journal of Molecular and Cellular Cardiology. 1998;30(11):2281-2289.

72. Samali A, Cotter TG. Heat Shock Proteins Increase Resistance to Apoptosis. Experimental Cell Research. 1996;223(1):163-170.

73. Stephanou A, Brar B, Heads R, et al. Cardiotrophin-1 Induces Heat Shock Protein Accumulation in Cultured Cardiac Cells and Protects them from Stressful Stimuli. Journal of Molecular and Cellular Cardiology. 1998;30(4):849-855.

74. Brar BK, Stephanou A, Wagstaff MJD, et al. Heat Shock Proteins Delivered With a Virus Vector Can Protect Cardiac Cells Against Apoptosis As Well As Against Thermal or Hypoxic Stress. Journal of Molecular and Cellular Cardiology. 1999;31(1):135-146.

75. Li C-Y, Lee J-S, Ko Y-G, Kim J-I, Seo J-S. Heat Shock Protein 70 Inhibits Apoptosis Downstream of Cytochrome c Release and Upstream of Caspase-3 Activation. Journal of Biological Chemistry. 2000;275(33):25665-25671.

76. Concannon CG, Orrenius S, Samali A. Hsp27 Inhibits Cytochrome c-Mediated Caspase Activation by Sequestering Both Pro-caspase-3 and Cytochrome c. Gene Expression. 2001;9(4\&5):195-201.

77. Bruey J-M, Ducasse C, Bonniaud P, et al. Hsp27 negatively regulates cell death by interacting with cytochrome c. Nature Cell Biology. 2000;2(9):645-652.

78. de Jong WW, Caspers G-J, Leunissen JAM. Genealogy of the $\alpha$-crystallin-small heatshock protein superfamily. International Journal of Biological Macromolecules.22(34):151-162.

79. Horwitz J. Alpha-crystallin can function as a molecular chaperone. Proceedings of the National Academy of Sciences. 1992;89(21):10449-10453.

80. Groenen PJTA, Merck KB, De Jong WW, Bloemendal H. Structure and Modifications of the Junior Chaperone $\alpha$-Crystallin. European Journal of Biochemistry. 1994;225(1):1-19.

81. Taylor RP, Benjamin IJ. Small heat shock proteins: a new classification scheme in mammals. Journal of Molecular and Cellular Cardiology. 2005;38(3):433-444.

82. Arrigo A-P, Simon S, Gibert B, et al. Hsp27 (HspB1) and $\alpha$ B-crystallin (HspB5) as therapeutic targets. FEBS Letters. 2007;581(19):3665-3674.

83. Klemenz R, Fröhli E, Steiger RH, Schäfer R, Aoyama A. Alpha B-crystallin is a small heat shock protein. Proceedings of the National Academy of Sciences. 1991;88(9):36523656.

84. Lin DI, Barbash O, Kumar KGS, et al. Phosphorylation-Dependent Ubiquitination of Cyclin D1 by the SCFFBX4- $\alpha$ B Crystallin Complex. Molecular Cell. 2006;24(3):355366. 
85. Launay N, Goudeau B, Kato K, Vicart P, Lilienbaum A. Cell signaling pathways to $\alpha \mathrm{B}-$ crystallin following stresses of the cytoskeleton. Experimental Cell Research. 2006;312(18):3570-3584.

86. Thériault JR, Lambert H, Chávez-Zobel AT, Charest G, Lavigne P, Landry J. Essential Role of the NH2-terminal WD/EPF Motif in the Phosphorylation-activated Protective Function of Mammalian Hsp27. Journal of Biological Chemistry. 2004;279(22):2346323471.

87. Morrison LE, Hoover HE, Thuerauf DJ, Glembotski CC. Mimicking Phosphorylation of $\alpha B-C r y s t a l l i n$ on Serine-59 Is Necessary and Sufficient to Provide Maximal Protection of Cardiac Myocytes From Apoptosis. Circulation Research. 2003;92(2):203-211.

88. Berry V, Francis P, Reddy MA, et al. Alpha-B Crystallin Gene (CRYAB) Mutation Causes Dominant Congenital Posterior Polar Cataract in Humans. The American Journal of Human Genetics. 2001;69(5):1141-1145.

89. Vicart P, Caron A, Guicheney $\mathrm{P}$, et al. A missense mutation in the [agr]B-crystallin chaperone gene causes a desmin-related myopathy. Nature Genetics. 1998;20(1):92-95.

90. Selcen D, Engel AG. Myofibrillar myopathy caused by novel dominant negative $\alpha \mathrm{B}$ crystallin mutations. Annals of Neurology. 2003;54(6):804-810.

91. Liu M, Ke T, Wang Z, et al. Identification of a CRYAB Mutation Associated with Autosomal Dominant Posterior Polar Cataract in a Chinese Family. Investigative Ophthalmology \& Visual Science. 2006;47(8):3461-3466.

92. Muchowski PJ, Wacker JL. Modulation of neurodegeneration by molecular chaperones. Nature Review Neuroscience. 2005;6(1):11-22.

93. Gruvberger-Saal SK, Parsons R. Is the small heat shock protein $\alpha$ B-crystallin an oncogene? Journal of Clinical Investigation. 2006;116(1):30-32.

94. Brady JP, Garland DL, Green DE, Tamm ER, Giblin FJ, Wawrousek EF. $\alpha$ B-crystallin in lens development and muscle integrity: A gene knockout approach. Investigative Ophthalmology and Visual Science. 2001;42(12):2924-2934.

95. Morrison LE, Whittaker RJ, Klepper RE, Wawrousek EF, Glembotski CC. Roles for $\alpha \mathrm{B}$ crystallin and HSPB2 in protecting the myocardium from ischemia-reperfusion-induced damage in a KO mouse model. American Journal of Physiology - Heart and Circulatory Physiology. 2004;286(3 55-3):H847-H855.

96. Ray PS, Martin JL, Swanson EA, Otani H, Dillman WH, Das DK. Transgene overexpression of $\alpha \mathrm{B}$ crystallin confers simultaneous protection against cardiomyocyte apoptosis and necrosis during myocardial ischemia and reperfusion. The Federation of American Societies for Experimental Biology Journal. 2001;15(2):393-402. 
97. Mao YW, Liu JP, Xiang H, Li DWC. Human [alpha]A- and [alpha]B-crystallins bind to Bax and Bcl-XS to sequester their translocation during staurosporine-induced apoptosis. Cell Death and Differentiation. 2004;11(5):512-526.

98. Kamradt MC, Chen F, Sam S, Cryns VL. The Small Heat Shock Protein $\alpha$ B-crystallin Negatively Regulates Apoptosis during Myogenic Differentiation by Inhibiting Caspase3 Activation. Journal of Biological Chemistry. 2002;277(41):38731-38736.

99. Bennardini F, Wrzosek A, Chiesi M. Alpha B-crystallin in cardiac tissue. Association with actin and desmin filaments. Circulation Research. 1992;71(2):288-294.

100. Martindale JJ, Wall JA, Martinez-Longoria DM, et al. Overexpression of Mitogenactivated Protein Kinase Kinase 6 in the Heart Improves Functional Recovery from Ischemia in Vitro and Protects against Myocardial Infarction in Vivo. Journal of Biological Chemistry. 2005;280(1):669-676.

101. Ito $\mathrm{H}$, Okamoto $\mathrm{K}$, Nakayama $\mathrm{H}$, Isobe $\mathrm{T}$, Kato $\mathrm{K}$. Phosphorylation of $\alpha \mathrm{B}$-Crystallin in Response to Various Types of Stress. Journal of Biological Chemistry. 1997;272(47):29934-29941.

102. Hoover HE, Thuerauf DJ, Martindale JJ, Glembotski CC. $\alpha$ B-crystallin Gene Induction and Phosphorylation by MKK6-activated p38. Journal of Biological Chemistry.2000 2000;275(31):23825-23833.

103. Mao Y-W, Xiang H, Wang J, Korsmeyer S, Reddan J, Li DW-C. Human bcl-2 Gene Attenuates the Ability of Rabbit Lens Epithelial Cells against H2O2-induced Apoptosis through Down-regulation of the $\alpha \mathrm{B}$-crystallin Gene. Journal of Biological Chemistry. 2001;276(46):43435-43445.

104. Kamradt MC, Chen F, Cryns VL. The Small Heat Shock Protein $\alpha$ B-Crystallin Negatively Regulates Cytochrome c- and Caspase-8-dependent Activation of Caspase-3 by Inhibiting Its Autoproteolytic Maturation. Journal of Biological Chemistry. May 11, 2001 2001;276(19):16059-16063.

105. Liu S, Li J, Tao Y, Xiao X. Small heat shock protein $\alpha$ B-crystallin binds to p53 to sequester its translocation to mitochondria during hydrogen peroxide-induced apoptosis. Biochemical and Biophysical Research Communications. 2007;354(1):109-114.

106. Bousette N, Chugh S, Fong V, et al. Constitutively active calcineurin induces cardiac endoplasmic reticulum stress and protects against apoptosis that is mediated by $\alpha$ crystallin-B. Proceedings of the National Academy of Sciences. 2010;107(43):1848118486.

107. MacLennan DH, Kranias EG. Phospholamban: a crucial regulator of cardiac contractility. Nat Rev Mol Cell Biol. 2003;4(7):566-577.

108. Gramolini AO, Kislinger T, Alikhani-Koopaei R, et al. Comparative Proteomics Profiling of a Phospholamban Mutant Mouse Model of Dilated Cardiomyopathy Reveals 
Progressive Intracellular Stress Responses. Molecular \& Cellular Proteomics. 2008;7(3):519-533.

109. Sharma P. IV, Grace K. , Ursprung C. , Kislinger T. , and Gramolini A.O. . Endoplasmic Reticulum Protein Targeting of Phospholamban: A Common Role for an N-Terminal DiArginine Motif in ER Retention? Public Library of Science One 2010;5(7).

110. Hess ML, Manson NH, Okabe E. Involvement of free radicals in the pathophysiology of ischemic heart disease. Canadian Journal of Physiology and Pharmacology. 1982;60(11):1382-1389.

111. Vandeplassche G, Hermans C, Thoné F, Borgers M. Mitochondrial hydrogen peroxide generation by NADH-oxidase activity following regional myocardial ischemia in the dog. Journal of Molecular and Cellular Cardiology. 1989;21(4):383-392.

112. Slezak Jea. Hydrogen Peroxide Changes in Ischemicand Reperfused Heart. American Journal of Pathology. 1995;147(3):772-781.

113. Longoni SJ, Chiesi M Cardiac alpha-crystallin. I. Isolation and identification. Molecular and Cellular Biochemistry. 1990;99(1):113-120.

114. Jin JK, Whittaker R, Glassy MS, Barlow SB, Gottlieb RA, Glembotski CC. Localization of phosphorylated $\hat{\mathrm{I}} \pm \mathrm{B}$-crystallin to heart mitochondria during ischemia-reperfusion. Vol 2942008:H337-H344.

115. Jin J-K, Whittaker R, Glassy MS, Barlow SB, Gottlieb RA, Glembotski CC. Localization of phosphorylated $\alpha \mathrm{B}$-crystallin to heart mitochondria during ischemia-reperfusion. American Journal of Physiology - Heart and Circulatory Physiology. 2008;294(1):H337$\mathrm{H} 344$.

116. He L, Lemasters JJ. Regulated and unregulated mitochondrial permeability transition pores: a new paradigm of pore structure and function? FEBS Letters. 2002;512(1):1-7.

117. Downs CA, Jones LR, Heckathorn SA. Evidence for a Novel Set of Small Heat-Shock Proteins That Associates with the Mitochondria of Murine PC12 Cells and Protects NADH:Ubiquinone Oxidoreductase from Heat and Oxidative Stress. Archives of Biochemistry and Biophysics. 1999;365(2):344-350.

118. Maloyan A, Sanbe A, Osinska H, et al. Mitochondrial Dysfunction and Apoptosis Underlie the Pathogenic Process in $\alpha$-B-Crystallin Desmin-Related Cardiomyopathy. Circulation. 2005;112(22):3451-3461.

119. Ide $\mathrm{T}$, Tsutsui $\mathrm{H}$, Kinugawa $\mathrm{S}$, et al. Mitochondrial Electron Transport Complex I Is a Potential Source of Oxygen Free Radicals in the Failing Myocardium. Circulation Research. 1999;85(4):357-363.

120. Schon EA, Manfredi G. Neuronal degeneration and mitochondrial dysfunction. The Journal of Clinical Investigation. 2003;111(3):303-312. 
121. Green DR, Kroemer G. The Pathophysiology of Mitochondrial Cell Death. Science. 2004;305(5684):626-629.

122. Zorov DB, Filburn CR, Klotz L-O, Zweier JL, Sollott SJ. Reactive Oxygen Species (RosInduced) Ros Release. The Journal of Experimental Medicine. 2000;192(7):1001-1014.

123. Wang X, Robbins J. Heart Failure and Protein Quality Control. Circulation Research. 2006;99(12):1315-1328.

124. Kamradt MC CF, and Cryns VL. . The Small Heat Shock Protein $\alpha$ B-Crystallin Negatively Regulates Cytochrome c- and Caspase-8-dependent Activation of Caspase-3 by Inhibiting Its Autoproteolytic Maturation. . Journal of Biological Chemistry. 2001;276: 16059-16063, .

125. Nakagawa T, Zhu H, Morishima N, et al. Caspase-12 mediates endoplasmic-reticulumspecific apoptosis and cytotoxicity by amyloid-[beta]. Nature. 2000;403(6765):98-103.

126. Wang F, He Q, Sun Y, Dai X, Yang X-P. Female Adult Mouse Cardiomyocytes Are Protected Against Oxidative Stress. Hypertension. 2010;55(5):1172-1178.

127. Elmore S. Apoptosis: A Review of Programmed Cell Death. Toxicologic Pathology. 2007;35(4):495-516.

128. Grey JY, Connor MK, Gordon JW, Yano M, Mori M, Hood DA. Tom20-mediated mitochondrial protein import in muscle cells during differentiation. American Journal of Physiology - Cell Physiology. 2000;279(5):C1393-C1400. 


\section{APPENDIX: ADDITIONAL DATA}

\subsection{Figures}

A

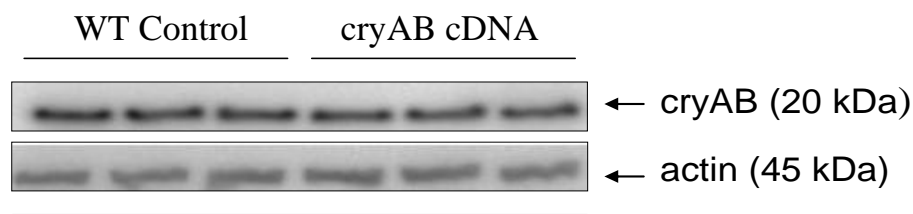

$\mathrm{OE}$

B

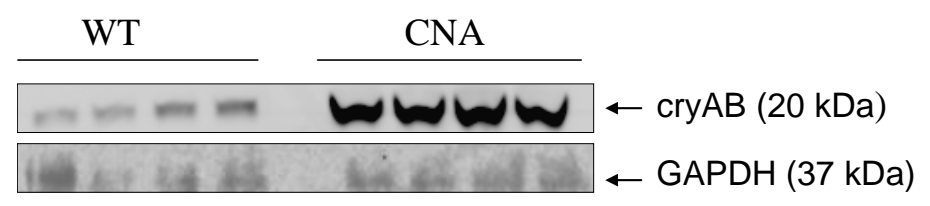

Figure 10. CryAB expression following over-expression and in CNA CMs.

(A) Immunoblots of cyAB and actin standard in either WT CMs (WT Control) or CMs transfected with cryAB cDNA (cryAB cDNA). cryAB expression levels were $93 \pm 4 \%$ relative to expression levels in WT control CMs. (B) Immunoblots for cryAB and standard GAPDH in WT and CNA adult mouse hearts. There was a $\sim 4.3$-fold increase in the expression of cryAB in the CNA hearts when compared to WT hearts $(\mathrm{p}<0.05)$. 

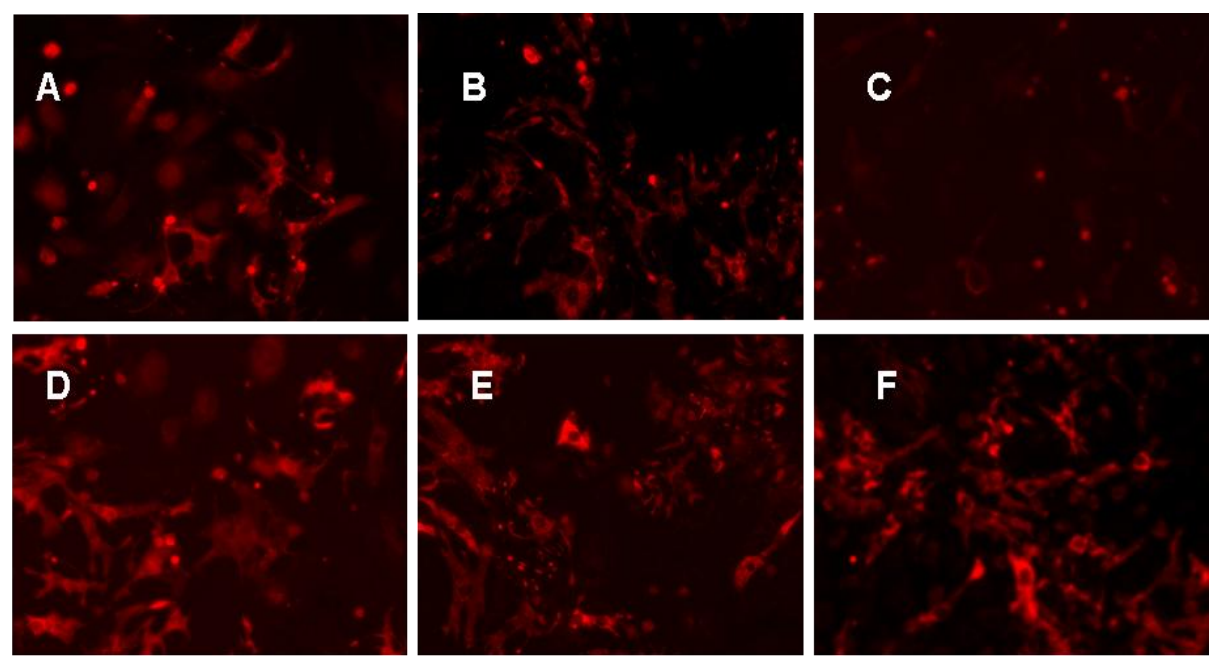

\section{Figure 11. Mitotracker retention in CNA cardiomyocytes.}

Immunoflourescent images showing loss of MitoTraker fluorescence in WT cardiomyocytes 24 hours following exposure to $200 \mu \mathrm{M} \mathrm{H}_{2} \mathrm{O}_{2}$. (A) WT cardiomyocytes, Time $0,0 \mu \mathrm{M} \mathrm{H}_{2} \mathrm{O}_{2}$. (B) WT cardiomyocytes, Time 24 hrs, 0 $\mu \mathrm{M} \mathrm{H}_{2} \mathrm{O}_{2}$. (C) WT cardiomyocytes, Time $24 \mathrm{hrs}, 200 \mu \mathrm{M} \mathrm{H}_{2} \mathrm{O}_{2}$. (D) CNA cardiomyocytes, Time $0,0 \mu \mathrm{M} \mathrm{H}_{2} \mathrm{O}_{2}$. (E) CNA cardiomyocytes, Time 24 hrs, $0 \mu \mathrm{M} \mathrm{H}_{2} \mathrm{O}_{2}$. (F) CNA cardiomyocytes, Time 24 hrs, $200 \mu \mathrm{M} \mathrm{H}_{2} \mathrm{O}_{2}$. 
A
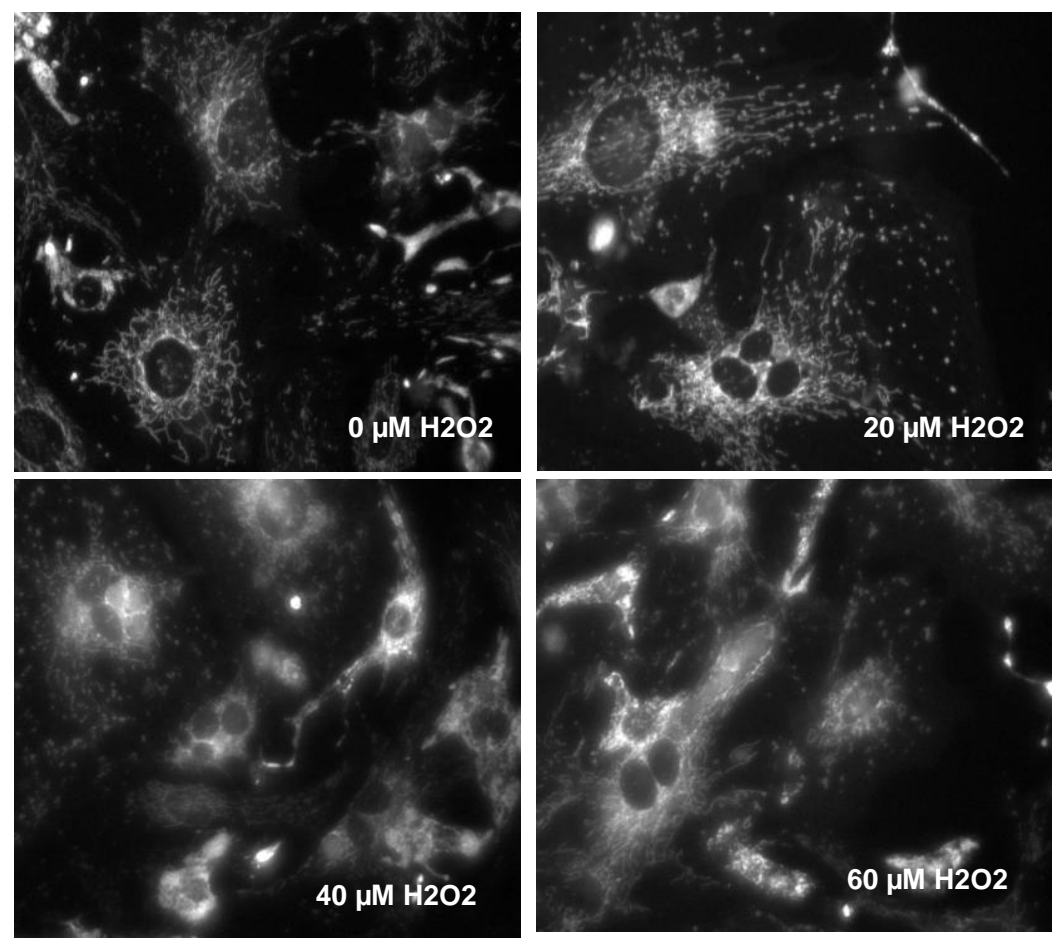

B

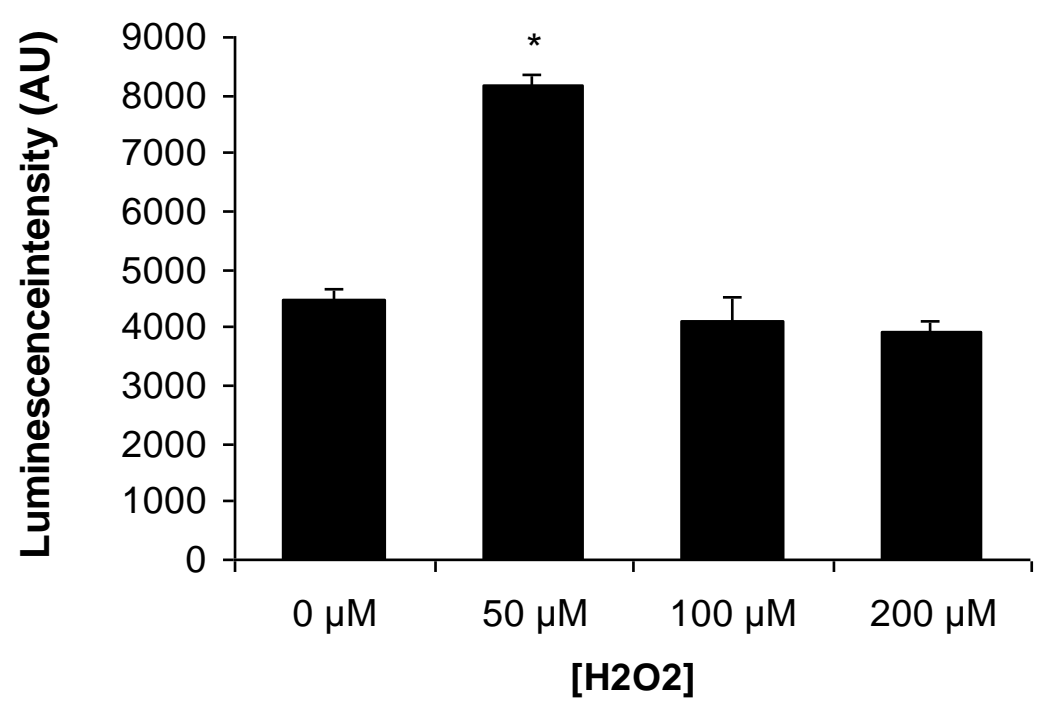

Figure 12. Optimization of $\mathrm{H}_{2} \mathrm{O}_{2}$ concentration for induction of apoptosis in CMs.

(A) Immunofluorescent images (20X) of $\mathrm{DiOC6}_{(3)}$ staining of mitochondria in $\mathrm{CMs}$ under control conditions and following exposure to increasing $\mathrm{H}_{2} \mathrm{O}_{2}$ concentrations (0 to $60 \mu \mathrm{M} \mathrm{H}_{2} \mathrm{O}_{2}$ ). (B) Quantification of luminescence intensity, indicative of caspase 3 activity in CMs at 0, 50, 100, $200 \mu \mathrm{M} \mathrm{H}_{2} \mathrm{O}_{2}$ (* at $\mathrm{p}<0.05$, following an analysis of variance and post-hoc Tukey test, $\mathrm{n}=6$ replicates/treatment). Values are represented as means $+\mathrm{SE}$. 
A

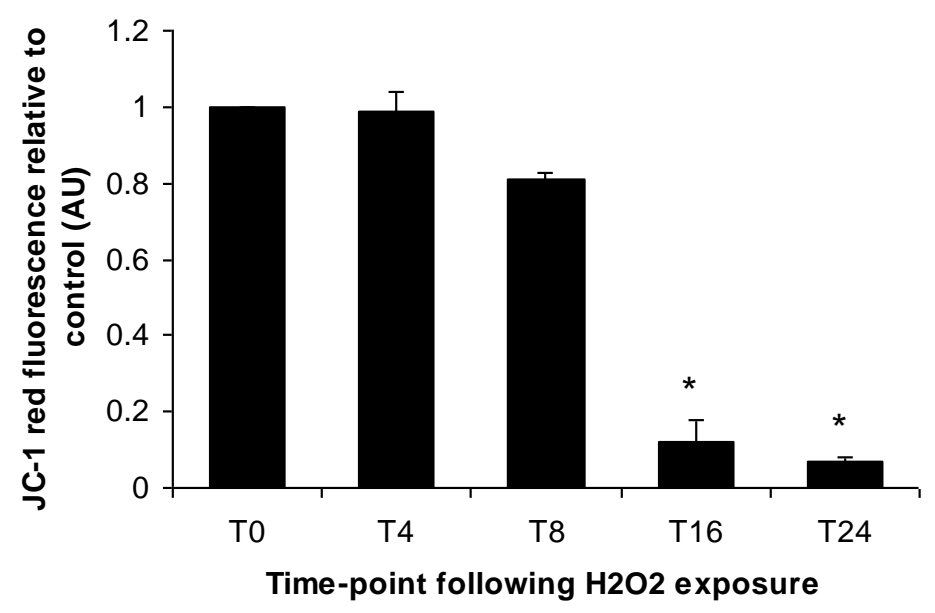

B

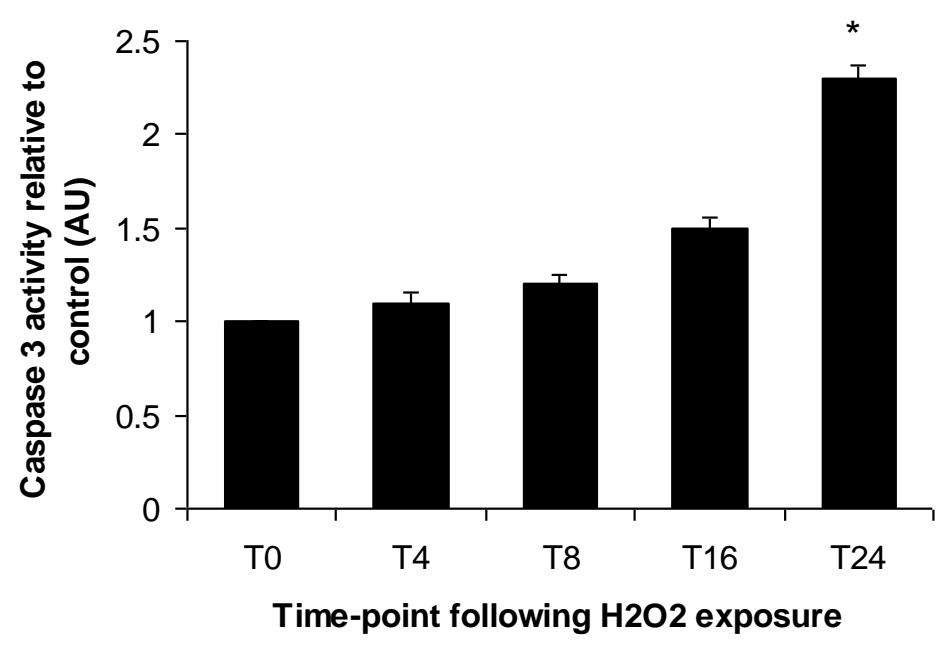

Figure 13. Time dependent dissipation of mitochondrial membrane potential and caspase 3 activity.

(A) Quantification of JC-1 red fluorescence following exposure to $60 \mu \mathrm{M} \mathrm{H}_{2} \mathrm{O}_{2}$ at 4, 8, 16, and 24 hours (T4, T8, T16, T24) following exposure compared to reference values set to $1 \mathrm{AU}$ under control conditions (T0) in cultured neonaltal CMs (*at $\mathrm{p}<0.05$ following ANOVA and Tukey post-hoc test). (B) Quantification of caspase 3 activity following exposure to $60 \mu \mathrm{M} \mathrm{H}_{2} \mathrm{O}_{2}$ at 4, 8, 16, and 24 hours following exposure(T4, T8, T16, T24) compared to reference values set to $1 \mathrm{AU}$ under control conditions (T0) in cultured neonaltal CMs (*at $\mathrm{p}<0.05$ following ANOVA and Tukey post-hoc test). Values are reported as means $+\mathrm{SE}$ ( $\mathrm{n}=4$ replicates/treatment). 
A
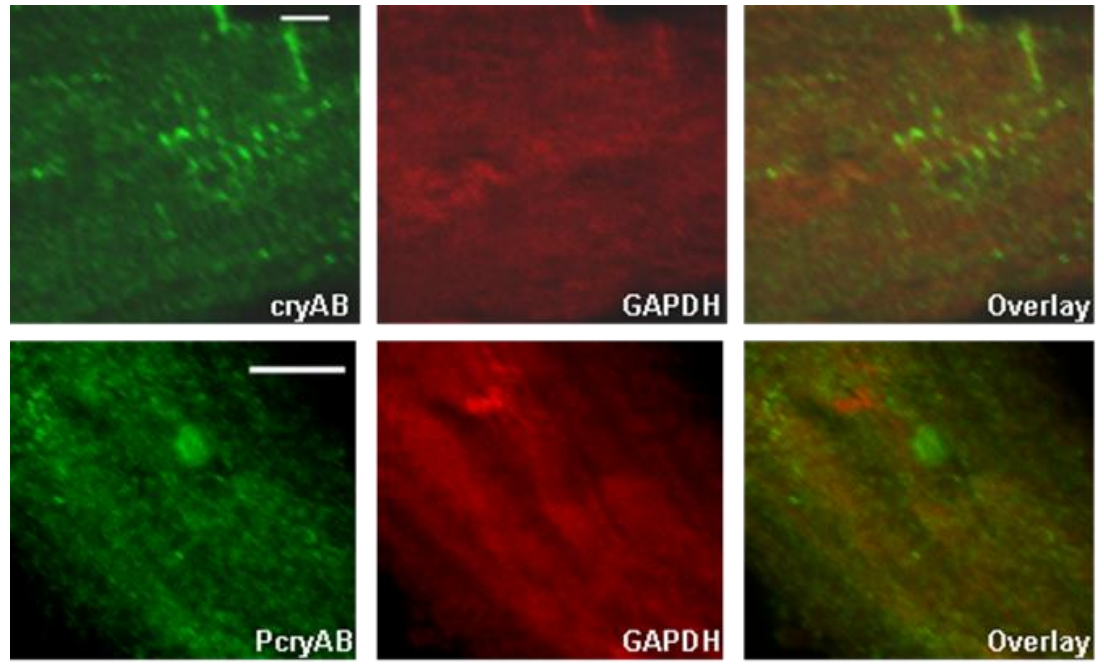

\section{Control}

B
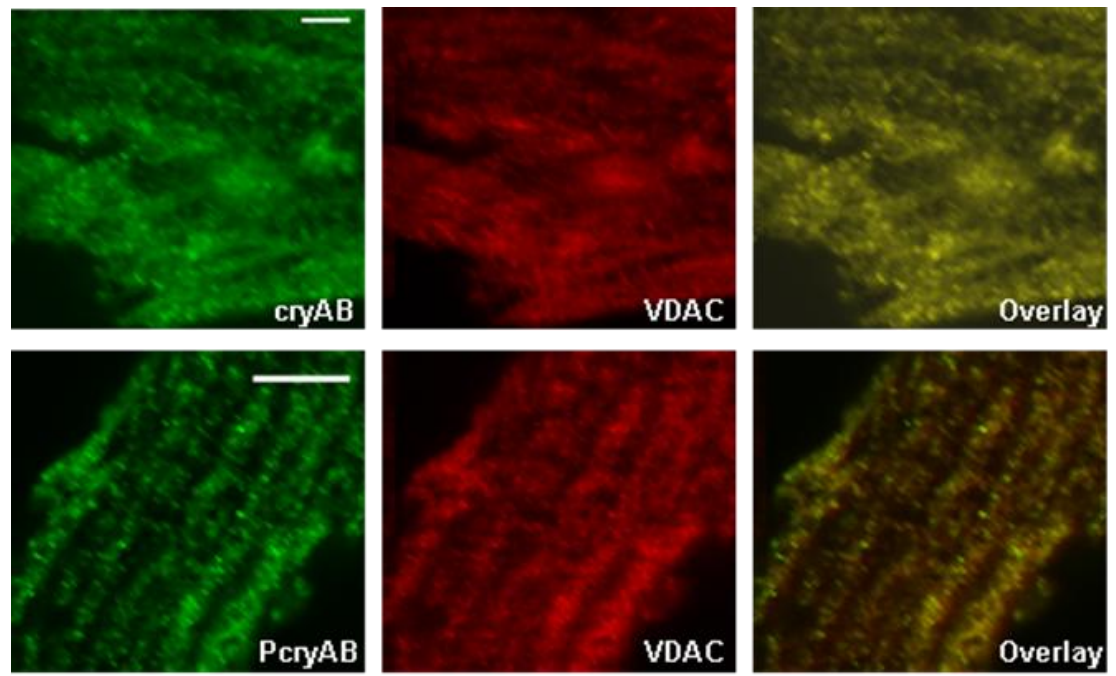

\section{$100 \mu \mathrm{M} \mathrm{H} \mathrm{O}_{2}$}

Figure 14. Translocation of cryAB and PcryAB to the mitochondria under oxidative stress conditions.

(A) Immunofluorescent images of cry $\mathrm{AB}$ and Pcry $\mathrm{AB}$ distribution in adult mouse $\mathrm{CMs}$ obtained by confocal microscopy under control conditions and (B) under stress conditions $\left(100 \mu \mathrm{M} \mathrm{H}_{2} \mathrm{O}_{2}\right)$. CryAB and PcryAB staining is depicted in green, GAPDH, a cytosolic marker, and VDAC, a mitochondrial marker, in red; cale bar is $4 \mu \mathrm{M}$. 
A
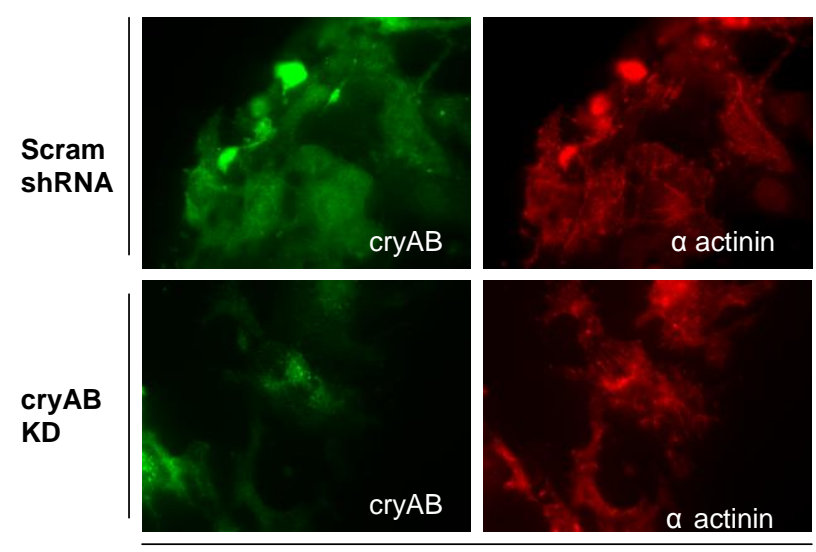

Control
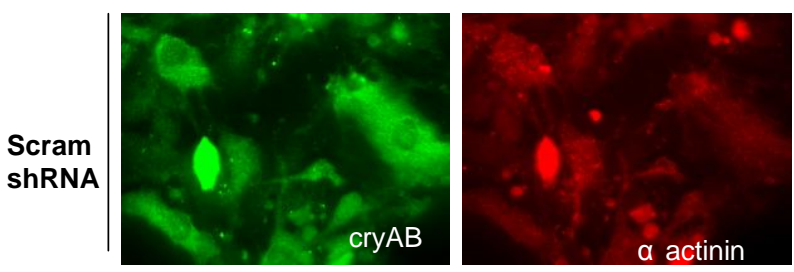

B
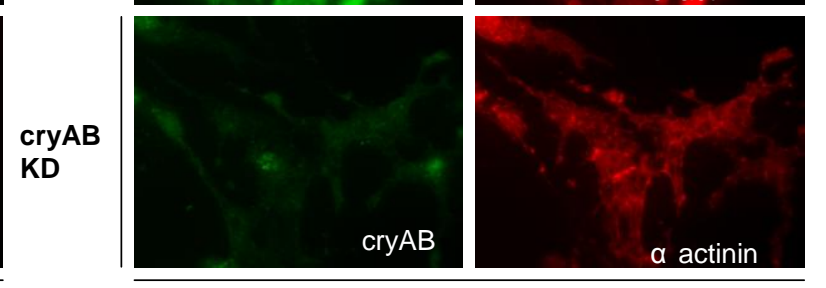

$60 \mu \mathrm{M} \mathrm{H}_{2} \mathrm{O}_{2}$

Figure 15. CryAB expression following stress and knock-down.

Immunofluorescent images (63X) of mouse neonatal CMs transduced with either lentiviruses containing scrambled shRNA( Scram shRNA) or shRNA targeting cryAB (cryAB KD) under (A) control conditions and (B) following exposure to $60 \mu \mathrm{M} \mathrm{H}_{2} \mathrm{O}_{2}$. CryAB staining is depicted in green and $\alpha$-actinin, a cardiomyocytes marker, in red. 


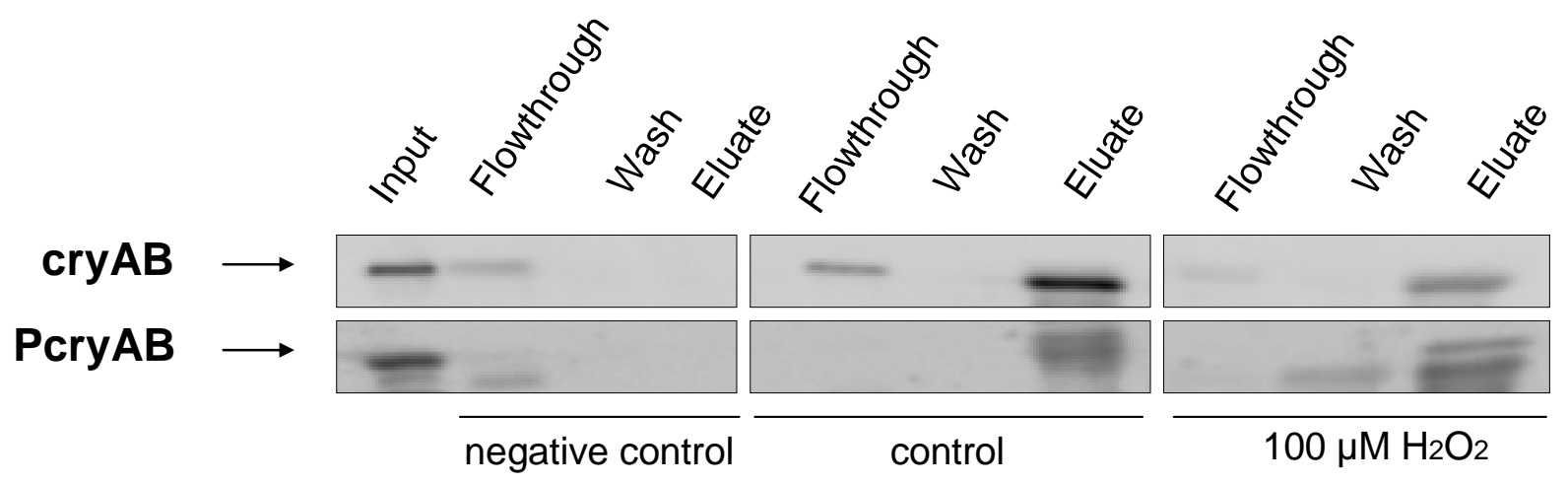

Figure 16. Co-immunoprecipitation of cryAB and cryAB.

Immunoblot depicting the enrichment of cryAB (top) and PcryAB (bottom) in the eluate and depletion in the flowthrough. I is input, -FT is flowthrough for the negative comtrol, -W is wash for negative control, - $\mathrm{E}$ is eluate for negative control. FTc is flowthrough for control, Wc is wash for control and Ec is eluate for control. FTs is flowthrough for stressed cells $\left(60 \mu \mathrm{M} \mathrm{H}_{2} \mathrm{O}_{2}\right)$, Ws is wash for stressed cells and Es is eluate for stressed cells. 
A

IP: cryAB

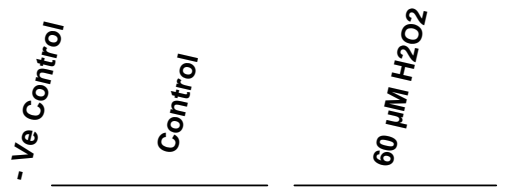

cryAB $\longrightarrow$

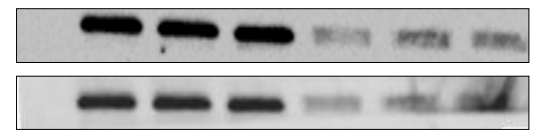

C

IP: cyto C

$\frac{K D}{E F T} \frac{\text { Control }}{E F T} \frac{\text {-ve }}{E F T}$

cyto $\mathrm{C} \longrightarrow$

caspase $9 \longrightarrow$

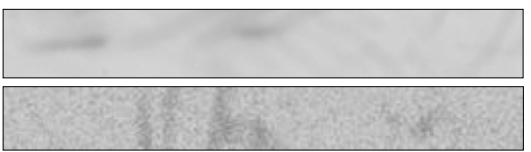

B

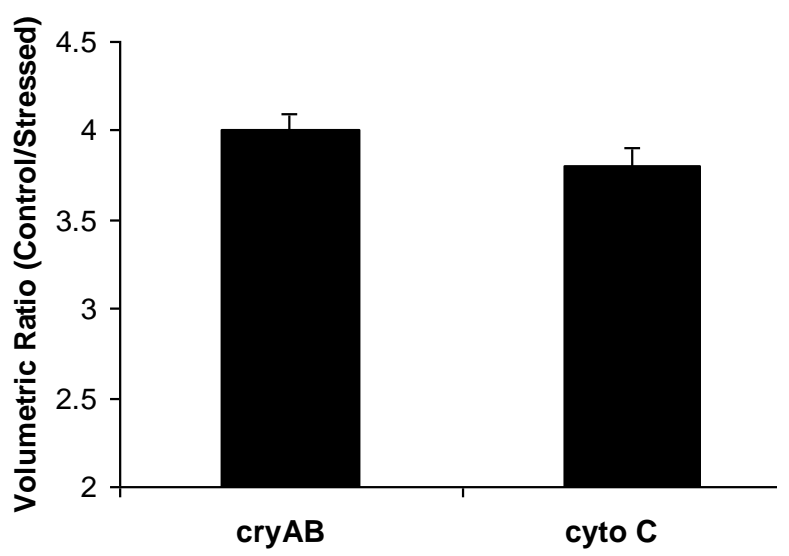

Figure 17. Interaction of cryAB with cytochrome $C$.

(A) Immunoblot depicting the presence of an interaction of cryAB with cytochrome $\mathrm{C}$ following immunoprecipitation of cryAB out of neonatal CMs. CryAB is loaded as a control. (B) Bar graph depicting no significant difference between the ratio of cryAB control to cryAB $60 \mu \mathrm{M} \mathrm{H}_{2} \mathrm{O}_{2}$ loaded to the ratio of cytochrome $\mathrm{C}$ control to cytochrome $\mathrm{C} 60 \mu \mathrm{M} \mathrm{H}_{2} \mathrm{O}_{2}$ loaded. (C) Immunoblot depicting no interaction between cytochrome $\mathrm{C}$ and caspase 9, following immunoprecipitation of cytochrome C. Probing with cytochrome $\mathrm{C}$ was used as a control. (Eeluate, FT-flowthrough). 
IP: cryAB

$$
\begin{array}{r}
\text { cryab }(20 \mathrm{kDa}) \\
\text { TOM20 }(20 \mathrm{kDa}) \\
\text { cytochrome c }(12 \mathrm{kDa}) \\
\text { caspase } 9(46 \mathrm{kDa})
\end{array}
$$

IP: PcryAB
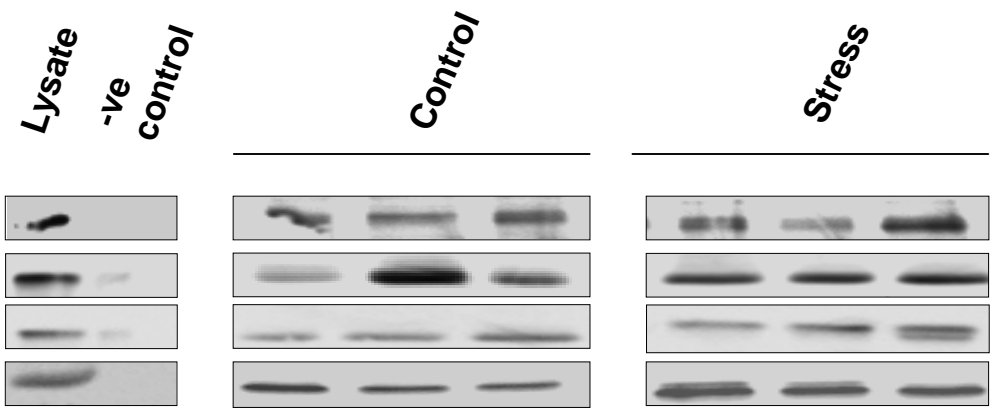

$$
\begin{array}{r}
\text { Pcryab }(22 \mathrm{kDa}) \\
\text { TOM20 }(20 \mathrm{kDa}) \\
\text { cytochrome c }(12 \mathrm{kDa}) \\
\text { caspase } 9(46 \mathrm{kDa})
\end{array}
$$
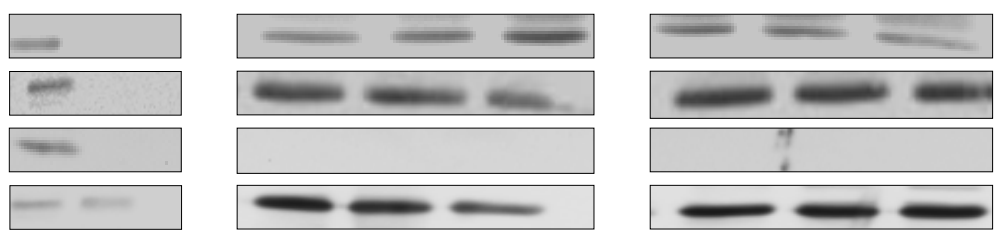

\section{Figure 18. CryAB interactions in the apoptotic cascade that do not change following exposure to stress.}

Immunoblots depicting the interactions of cryAB and PcryAB with TOM 20, cytochrome C, and caspase 9 . CryAB and PcryAB were immunoprecipitated from control and stressed adult cardiac tissue $\left(100 \mu \mathrm{M} \mathrm{H}_{2} \mathrm{O}_{2}\right.$ for 1 hour $)$ using $\mathrm{A} / \mathrm{G}$ agarose beads and the eluate was run on an SDS-page gel, probing for interactors in both control and stressed cardiac tissue. The gels were also probed for cryAB and PcryAB as a control. IgG beads alone were used as negative controls. Equal loading of cardiac lysate is shown. 


\subsection{Results and Discussion}

\subsubsection{Over-expression of cryAB in mouse neonatal CMs}

In order to validate the protective properties of cryAB against apoptosis that we initially uncovered $^{106}$, I over-expressed cryAB in cultured mouse neonatal CMs, using using lentivector mediated transduction of cryAB cDNA. A scrambled control was also used. Lentivector production and transduction of neonatal CMs was carried out as described in Chapter 2. The success of the over-expression transduction was validated via immunoblot analysis. The overexpression did not lead to upregulation of cryAB in the cardiomyocytes (Fig. 10). Following transduction, cryAB expression levels were $93 \pm 4 \%$ relative to expression levels in control CMs (Fig. 10). Probing for actin was used as a control and it shows a uniform distribution in the WT CMs and the CMs transduced with the cryAB cDNA construct.

CNA cardiomyocytes in which cryAB is upregulated were as an alternative to the overexpression model. The upregulation of cryAB was validated by Western Blot analysis of protein isolated from adult whole heart lysates (Fig. 10). There was a $\sim 4$.3-fold increase in the expression of cryAB in the CNA hearts when compared to WT hearts (expression levels of $425 \pm$ $40 \%$ in CNA hearts compared to $100 \%$ levels observed in WT hearts; $p<0.05$ ).

\subsubsection{Protection against apoptosis}

To determine whether upregulation of cryAB was protective against apoptosis in CNA CMs, WT and CNA CMs were exposed to $200 \mu \mathrm{M} \mathrm{H}_{2} \mathrm{O}_{2}$ and Mitotracker fluorescence was examined. Mitotracker is a cell-permeable dye that accumulates in active mitochondria, where it becomes oxidized and fluorescent. Mitotracker fluorescence thus gives an indication of actively respiring cells, or live cells. Upon exposure of CNA and WT CMs to $200 \mu \mathrm{M} \mathrm{H}_{2} \mathrm{O}_{2}$, the CNA CMs retained Mitotracker fluorescence after 24 hours, whereas WT CMs seemed to have lost almost all mitochondrial staining (Fig. 11). This suggests that CNA CMs are protected against $\mathrm{H}_{2} \mathrm{O}_{2-}$ induced apoptosis. However, it was not possible to single out cryAB as a mediator of this effect, due to the many other alterations in CNA CMs when compared to WT CMs. Future experiments focused only on $\mathrm{CMs}$ in which cryAB had been silenced (KD cryAB). 


\subsubsection{Optimization of $\mathrm{H}_{2} \mathrm{O}_{2}$ concentration for the induction of apoptosis}

To determine the optimal concentration that would induce CM death, WT CMs were exposed to varying levels of $\mathrm{H}_{2} \mathrm{O}_{2}$ and mitochondrial integrity was examined by $\operatorname{DiOC6}_{(3)}$ staining, an indicator of mitochondrial membrane potential. Dissipation of mitochondrial membrane potential leads to the rupture of the outer mitochondrial membrane, leading to apoptosis. Caspase 3 activity was also evaluated, also an indicator of apoptosis. DiOC6 $(3)$ staining became more diffuse with increasing $\mathrm{H}_{2} \mathrm{O}_{2}$ concentrations and the signal became faintest at $60 \mu \mathrm{M} \mathrm{H}_{2} \mathrm{O}_{2}$, indicating that the mitochondria in those cells was compromised, which would subsequently lead to apoptosis (Fig. 12A). This optimal concentration for the induction of apoptosis was corroborated by the caspase 3 activity assay at different $\mathrm{H}_{2} \mathrm{O}_{2}$ concentrations. Caspase 3 activity was maximal (1.9-fold higher than observed in controls, $8260 \pm 102$ A.U. vs. $4347 \pm 120$ A.U. ) at $50 \mu \mathrm{M}$ ( ANOVA, follwwed by post-hoc Tukey, * at p < 0.05), after which there was a decline in activity to below control levels, $4100 \pm 464$ A.U. at $100 \mu \mathrm{M} \mathrm{H}_{2} \mathrm{O}_{2}$ and $3934 \pm 132$ at $200 \mu \mathrm{M}$ $\mathrm{H}_{2} \mathrm{O}_{2}$ (Fig. 12B). This could be explained by the fact that some of the cells were already dead at concentrations higher than $50 \mu \mathrm{M}$, leaving fewer cells to give off luminescence. From here on out I used a concentration of $60 \mu \mathrm{M} \mathrm{H}_{2} \mathrm{O}_{2}$ to stress the isolated neonatal CMs, as this seemed to be the optimal concentration that induced apoptosis and $100 \mu \mathrm{M} \mathrm{H}_{2} \mathrm{O}_{2}$ for adult CMs, as described previously ${ }^{126}$.

\subsubsection{Optimization of time-points for apoptosis measurements}

Apoptosis can be measured by a number of indicators and at different stages. Indicators of early apoptosis include dissipation of mitochondrial membrane potential ${ }^{127}$. The electrochemical gradient across the mitochondrial outer membrane (MOM) collapses during apoptosis, allowing detection with a fluorescent cationic dye, JC-1. In healthy cells this lipophilic dye accumulates in the mitochondria, forming aggregates that emit red fluorescence. In apoptotic cells the MOM does not maintain the electrochemical gradient and the cationic dye diffuses into the cytoplasm where it emits green fluorescence as a monomer. A shift from red to green fluorescence thus indicates depolarization, hence the loss of red fluorescence at its maximal point was important to determine apoptosis. The time-point when caspase 3 activity, an executioner caspase and

indicator of late-stage apoptosis ${ }^{127}$ was maximal was also investigated. There was a progressive decrease in JC-1 red fluorescence which was significantly lower than control values, set to reference levels of 1 A.U. at 16 hours post exposure to $60 \mu \mathrm{M} \mathrm{H}_{2} \mathrm{O}_{2}$, dropping to one tenth the 
value observed in controls $(0.12 \pm 0.01$ A.U. relative to reference levels of 1 A.U. observed in controls at time 0 ; analysis of variance and Tukey post-hoc test, * at p < 0.05). This did not decrease significantly at 24 hours (0.07 0.01 A.U.) (Fig. 13A). Regarding caspase 3 activity, there was a progressive increase compared to control values with time (Fig. 13B). At 4 hours following exposure, I observed values of $1.1 \pm 0.01$ A.U., at 8 hours $1.2 \pm 0.01$ A.U., at 16 hours $1.5 \pm 0.02$ A.U., compared to control reference levels of 1 A.U. The activity reached maximal levels (2.3-fold increase compared to controls set at a reference level of 1 A.U.) at 24 hours post exposure to $60 \mu \mathrm{M} \mathrm{H}_{2} \mathrm{O}_{2}$ (analysis of variance and Tukey post-hoc test, * at $\mathrm{p}<0.05$ ). JC1fluorescence, or early apoptosis was thus measured at 16 hours and caspase 3 activity or late apoptosis at 24 hours post exposure to $60 \mu \mathrm{M} \mathrm{H}_{2} \mathrm{O}_{2}$ in subsequent experiments.

\subsubsection{Visual representation of cryAB localization and knock-down}

For a visual representation of the localization of cryAB and PcryAB in control and stressed (100 $\mu \mathrm{M} \mathrm{H}_{2} \mathrm{O}_{2}$ ) adult mouse CMs immunofluorescence was performed using a Leica TCS SP laser scanning confocal system. The isolated CMs were stained them with anti-cryAB or anti-PcryAB antibodies and antibodies against known organellar markers. CryAB and PcryAB were stained in green and VDAC and GAPDH in red. Figure 14A shows that cryAB displays a diffuse staining pattern, which is similar with the staining pattern of GAPDH, a known cytosolic marker. The far right upper panel shows the overlay of the two and yellow is representative of co-localization of cyAB with GAPDH. The images suggest that under control conditions cryAB is found in the cytosol, thus validating the previously obtained sub-cellular fractionation results. The lower panel of Figure 14A shows that PcryAB has a more aggregated and punctuated staining pattern and, which shows limited yellow overlay with GAPDH, suggesting a limited cytosolic localization. In Figure 14B, cryAB and PcryAB are co-stained with VDAC, a mitochondrial marker under stress conditions $\left(100 \mu \mathrm{M} \mathrm{H}_{2} \mathrm{O}_{2}\right)$. Under stress conditions, both cryAB and PcryAB display a punctuated staining pattern and significant yellow overlay with red VDAC (far right panels), suggesting a mitochondrial localization (Fig. 14B), further illustrating the translocation of cryAB from the cytosol to the mitochondria following exposure to oxidative stress. 
To validate the efficiency of the knock-down images of neonatal CMs following knock-down of cryAB are also presented. Figure 15A upper left panel depicts cryAB expression in CMs transduced with scrambled shRNA (Scram shRNA) under control conditions and $\alpha$-actinin staining, as a marker of CMs in the upper right panel. There was an increase in relative cryAB fluorescence following exposure to $60 \mu \mathrm{M} \mathrm{H}_{2} \mathrm{O}_{2}$ in the CMs transduced with scrambled shRNA (Fig. 15B - upper panels) and a significant reduction in cryAB fluorescence following transduction of the CMs with cryAB-targeting shRNA (cryAB KD) regardless of stress (fig. 15A-B, lower panels). Alpha-actinin levels remained constant regardless of stress or transduction (Fig.15).

\subsubsection{Identification of cryAB protective mechanism and binding interactors in the intrinsic apoptotic cascade}

In addition to the positive interactions of cryAB and PcryAB shown in Figure 8, other interactions were found as well. CryAB and Pcry $\mathrm{AB}$ were immunoprecipitated out of heart tissue homogenates from control hearts or hearts exposed to $100 \mu \mathrm{M} \mathrm{H}_{2} \mathrm{O}_{2}$ for 1 hour. Figure 16 shows the enrichment of cryAB and PcryAB in the eluate and depletion in the washes and floughthrough.

Figure 17 shows a preliminary attempt to determine an interaction of cryAB with cytochrome C. At this point, I was not able to measure concentrations for the eluate (likely because of the low $\mathrm{pH}$ ) and so cryAB was loaded as a control (Fig 17A). There was indeed a positive band for both control and stressed conditions when probing for cytochrome $\mathrm{C}$. When quantifying the ratio of cryAB control to cryAB $60 \mu \mathrm{M} \mathrm{H}_{2} \mathrm{O}_{2}$ loaded, it was not significantly different from the ration of cytochrome $\mathrm{C}$ control to cytochrome $\mathrm{C} 60 \mu \mathrm{M} \mathrm{H}_{2} \mathrm{O}_{2}$ loaded, which led to the conclusion that the interaction of cryAB with cytochrome $\mathrm{C}$ was similar under control conditions and following exposure to oxidative stress (Fig. 17B).

Since cryAB interacted with cytochrome C, I hypothesized that through this intecaction it may be possible that cryAB may prevent the interaction of cytochrome $\mathrm{C}$ with caspase 9 thus preventing apoptosome formation and inhibiting the progression of the apoptotic cascade. Therefore, I expected that in the absence of cryAB, the interaction of cytochrome $\mathrm{C}$ and caspase 9 would take place more readily. So I immunoprecipitated cytochrome C out of neonatal CMs cultured under normal conditions of CMs in which cryAB had been silenced (KD CMs), shown 
in Figure $17 \mathrm{C}$ by an enrichment of cytochrome $\mathrm{C}$ in the eluate when compared to the flowthrough in both control and KD cells. I probed for caspase 9, but found no positive bands. Since no positive control (lysate) was loaded, it is difficult to conclude whether no interaction was taking place or whether the absence of bands was due to antibody issues. Performing immunoprecipitation out of neonatal CMs was very difficult due to the very low protein yield and the limited number of pups that could be sacrificed for each trial. This was thus not pursued any further and immunoprecipitation was performed out of adult mouse whole heart lysates.

Co-immunoprecipitation experiments showed that cryAB and PcryAB both co-precipitated with mitochondrial protein TOM 20, an import receptor involved in importing preproteins into the mitochondria $^{128}$, and with caspase 9. CryAB and PcryAB formed complexes with TOM 20, which were increased 1.2-fold and 1.4-fold, respectively, under stressed conditions compared to control levels; and increased 1.4-fold and 1.5-fold with caspase 9. CryAB, but not PcryAB was found to precipitate with cytochrome $\mathrm{C}$ under both control and stress conditions at a 1:1 ratio (Fig. 18). These results suggest that the interaction of cryAB and PcryAB with TOM 20 and caspase 9 may not contribute to the protective mechanism of cryAB, as they are similar under control and stress conditions. The interaction of cryAB with cytochrome $\mathrm{C}$ is also similar under both control and stress conditions, suggesting that this interaction may also be inconsequential to its protective mechanism. Furthermore, there was no interaction of PcryAB with cytochrome C. This difference could be explained by the different distribution of cryAB and PcryAB. Following exposure to stress, some unphosphorylated would cryAB remain in the cytosol and would be capable of interacting with cytochrome $\mathrm{C}$ to prevent the progression of apoptosis. PcryAB levels, on the other hand, are expected to increase following stress when PcryAB associates mainly with the mitochondria. Following mitochondrial membrane rupture due to oxidative stress, cytochrome $\mathrm{C}$ would be found in the cytosol and PcryAB in the mitochondria, making an interaction between the two less likely than an interaction between unphosphorylated cryAB, some of which is still found in cytosol, as shown in Figure 5. 